\author{
Universidade de São Paulo \\ Instituto de Astronomia, Geofísica e Ciências Atmosféricas \\ Departamento de Astronomia
}

\title{
Origem e Evolução dos Campos Magnéticos Cosmológicos
}

Rafael da Silva de Souza

Orientador: Prof. Dr. Reuven Opher

Tese apresentada ao Departamento de Astronomia do Instituto de Astronomia, Geofísica e Ciências Atmosféricas da Universidade de São Paulo como parte dos requisitos para a obtenção do título de Doutor em Ciências.

Sub-área de Concentração: Astrofísica

São Paulo - Julho de 2009 
A astronomia é útil porque nos eleva acima de nós mesmos; é útil porque é grande, é útil porque é bela; isso é o que se precisa dizer. É ela que nos mostra o quanto o homem é pequeno no corpo e o quanto é grande no espírito, já que nesta imensidão resplandecente, onde seu corpo não passa de um ponto obscuro, sua inteligência pode abarcar inteira, e dela fluir a silenciosa harmonia. Atingimos assim a consciência de nossa força, e isso é uma coisa pela qual jamais pagaríamos caro demais, porque essa consciência nos torna mais fortes. (Em "O valor da ciência"de Henri Poincaré) 


\section{AGRADECIMENTOS}

Agradeço à realidade, que apesar de inacessível em sua essência. Nos serve como um guia através do qual prosseguimos em nossa jornada, buscando elucidar tão suaves e intricadas nuanças.

À minha querida família, meu pai Edson, minha mãe Rute, meu irmão Rodolfo, minhas tias Rosângela e Zuleica, por todo apoio ao longo do caminho trilhado até hoje.

Minha querida namorada Émille por todo apoio, cumplicidade e discussões altamente proveitosas sobre meu trabalho, além é claro de sua extremamente útil revisão da minha tese. Ao meus amigos do IAG, Tatiana, Mariana, Rodrigo, Luís Felipe, Gustavo, Alberto, Vinicius e Ulisses, pela convivência ao longos destes anos.

Aos meus amigos do Valongo, Rodolfo, Paulo e Tiago. Minha grande amiga Graziela pela convivência ao longo da minha jornada astronômica e pela grande ajuda na revisão da minha tese.

Aos meus amigos do Kung Fu, César, Selma, Arthur, Juliana, Fernando, Fausto, Nayra, Luisão, Cléber e tantos outros pela agradavél convivência ao longos destes anos.

Ao meu orientador de graduação Ioav Waga pela dedicação e apoio que sem dúvida alguma foram decisivas na minha etapa seguinte no IAG. Minha relatora Elisabete Dal Pino pelas inúmeras críticas e sugestões sempre proveitosas sobre nosso trabalho.

Ao meu orientador de doutorado Reuven Opher, com o qual foi uma grande honra e prazer trabalhar ao longo destes anos. Sua orientação e experiência foram 
decisivas na ampliação do minha visão da astrofísica, assim como seu amor não por alguma área em especial, mas pela ciência em sua totalidade. Agradeço ao suporte financeiro da Fapesp referente ao processo 04/05961-0. 


\section{RESUMO}

Campos magnéticos de intensidade $\sim \mu \mathrm{G}$ são observados tanto em nossa galáxia, quanto em galáxias com alto desvio para o vermelho $(z)$, onde o dínamo $\alpha-\Omega$ não deveria ter tempo para produzi-lo. Por conseguinte, uma origem primordial é indicada. Foi proposto que os campos primordiais surgiram em várias eras: durante a inflação, na transição de fase eletrofraca, na transição de fase quark hádron (TFQH), durante a formação dos primeiros objetos e durante a reionização. Nós sugerimos aqui, que estes campos magnéticos observados em galáxias através de medidas de rotação Faraday, têm sua origem em flutuações eletromagnéticas que naturalmente ocorreram no plasma quente e denso, existente logo após a TFQH. Nós evoluímos os campos previstos por nosso modelo até a época atual. O tamanho da região de coerência do campo magnético aumenta devido à fusão de regiões menores. Campos magnéticos de $\sim 10 \mu \mathrm{G}$ sobre regiões comóveis de $\sim 1$ pc foram encontrados para $z \sim 10$.

Investigamos a amplificação destes campos sementes pelo dínamo turbulento em protogaláxias. A taxa de amplificação devido à um vórtice turbulento de raio $L$ com velocidade circular $V$ é da ordem de $V / L$. Enquanto o modelo padrão de

dínamo tem um tempo de amplificação para um disco galáctico típico de $\sim 10^{9}$ anos, o dínamo turbulento de pequena escala tem uma taxa de amplificação de $\sim$ $10^{7}$ anos. Usamos as equações não-lineares para evolução da correlação magnética de forma a avaliar a evolução da amplificação destes campos na protogaláxia.

Vários autores sugeriram uma origem gravitacional para os campos magnéticos em objetos celestes em rotação. Isto foi motivado em parte pela conjectura Schuster-Blackett (S-B), onde se propõe que os campos magnéticos em planetas 
e estrelas surgem devido à sua rotação. Neste cenário, correntes de massa neutra geram campos magnéticos, implicando na existência de um acoplamento entre os campos gravitacional e magnético. Nós também investigamos a possibilidade da conjectura S-B ser a origem dos intensos campos magnéticos em magnetares e gamma ray bursts.

Além disso, estudamos a influência da pressões não térmicas, na determinação da massa de aglomerados de galáxias, usando dados públicos do XMM-Newton para 5 aglomerados de Abell. A pressão não térmica considerada aqui, é composta pelas componentes magnética e turbulenta. Nós consideramos estas duas componentes na equação do equilíbrio hidrostático e comparamos as estimativas de massa total, com os valores obtidos sem estas componentes.

Palavras-chave: Plasmas, Campos Magnéticos, Cosmologia, Turbulência, Aglomerados de Galáxias 


\begin{abstract}
Magnetic fields of intensities $\sim \mu \mathrm{G}$ are observed both in our galaxy and in high redshift $(z)$ galaxies, where a mean field dynamo would not had time to produce them. Therefore, a primordial origin is indicated. It has been suggested that magnetic fields were created at various primordial eras: during inflation, the electroweak phase transition, the quark-hadron phase transition (QHPT), during the formation of the first objects, and during reionization. We suggest here that the magnetic fields observed in galaxies by Faraday Rotation Measurements (FRMs), have their origin in the electromagnetic fluctuations that naturally occurred in the dense hot plasma that existed just after the QHPT. We evolve the predicted fields to the present time. The size of the region containing a coherent magnetic field increased due to the fusion of smaller regions. Magnetic fields (MFs) $\sim 10 \mu \mathrm{G}$ over a comoving $\sim 1 \mathrm{pc}$ region are predicted at redshift $z \sim 10$. The amplification of these seed fields by the turbulent dynamo in a protogalaxy is here investigated. The e-fold amplification time by a turbulent eddy of radius $L$ with a circular velocity $V$ is on the order of $L / V$. Whereas the standard dynamo for a typical disk galaxy has an e-fold amplification time $\sim 10^{9}$ years, the small scale turbulent dynamo has an e-fold time $\sim 10^{7}$ years. We use the non-linear evolution equations for the magnetic correlations in order to analyze the amplifications of these fields in protogalaxies. Various authors have suggested a gravitational origin of the magnetic fields in rotating celestial bodies. It has been motivated, in part, by the Schuster-Blackett (S-B) conjecture, which suggests that the magnetic fields in planets and stars arise due to their rotation. In this scenario, neutral mass currents generate magnetic fields, implying the existence of a coupling between gravitational
\end{abstract}


viii

and electromagnetic fields. In this work, we investigate the possibility that the S$\mathrm{B}$ conjecture is the origin of the intense magnetic fields near rotating compact objects, in particular connected with magnetars and gamma ray bursts.

We also studied the influence of non-thermal pressure on the cluster mass determination using public XMM-Newton archival data for 5 Abell clusters. The non-thermal pressure considered here, is composed of the magnetic and the turbulent components. We also take into account these two non-thermal components in the hydrostatic equilibrium equation, and we compare the total mass estimated with the values obtained without assuming them.

Key words: Plasmas, Magnetic Fields, Cosmology, Turbulence, Galaxy Clusters 


\section{Lista de Figuras}

3.1 Evolução do tempo de giro $\tau_{f l i p}$ (s) das bolhas magnéticas em função do tempot(s). . . . . . . . . . . . . . . . 43

3.2 Evolução do tempo de reconexão $\tau_{\text {tear }}$ (s) das bolhas magnéticas em função do tempo t(s). . . . . . . . . . . . . . . . . . . . 44 44

3.3 Razão entre o tempo de giro $\tau_{\text {flip }}$ e o tempo de reconexão $\tau_{\text {tear }}$ das bolhas magnéticas em função do tempo t(s). . . . . . . . . . . . 45

3.4 Razão entre o tempo de coalescência $\tau_{\text {coal }}$ e o tempo de difusão das bolhas magnéticas $\tau_{\text {diff }} \ldots \ldots \ldots \ldots \ldots \ldots$

3.5 Evolução inicial do tamanho físico das bolhas magnéticas, criadas imediatamente após a TFQH, como função do tempo, $t \equiv t_{0}+\Delta t$, para $t_{0}=10^{-4} \mathrm{~s}$, e $0<\Delta t\left(10^{-8} s\right) \leq 10 \ldots \ldots \ldots \ldots$

3.6 Evolução do tamanho físico das bolhas magnéticas em função do tempo t, a partir de $\sim 0.1 s . \ldots \ldots \ldots \ldots$

3.7 Evolução da intensidade do campo magnético $B(\mu \mathrm{G})$ nas bolhas, criado imediatamente após a TFQH, em função do tempo t(s). . . . 49 
3.8 Evolução da média do campo magnético $B(\mu G)$ ao longo da linha de visada, sobre uma região comóvel de $\sim 1$ Mpc como função do tempo $\mathrm{t}(\mathrm{s})$, desde $t \simeq 3 \times 10^{3}$ s até $t \sim 10^{16} \mathrm{~s}(z \sim 10)$, quando surgiram as primeiras galáxias. . . . . . . . . . . . 50

4.1 Valores de $B(k)$ como função do número de onda $k_{0}\left(k_{0}=2 \pi / L_{P G}\right)$ em diversos instantes de tempo. . . . . . . . . . . . . . 68

4.2 Valores de $M_{L}\left(G^{2}\right)$ como função do tempo t (anos). A curva preta contínua representa os valores de referência: $L_{c}=200 k p c, \mathrm{r}=3$ kpc, $V_{c}=10^{7} \mathrm{cms}^{-1}$ e $M_{L}(r, 0)=10^{-11}(0.1 p c / r)^{3} G^{2}$. Nós variamos r: curva vermelha tracejada $r=4 k p c$, curva azul ponto-traço-ponto $r=5 k p c \ldots \ldots \ldots \ldots \ldots \ldots$

4.3 Valores de $M_{L}\left(G^{2}\right)$ como função do tempo t(anos). A curva preta contínua representa os valores de referência (veja fig. 4.2). Nós variamos $V_{c}$ : curva vermelha tracejada $V_{c}=8 \times 10^{6} \mathrm{~cm} / \mathrm{s}$, curva azul ponto-traço-ponto $V_{c}=6 \times 10^{6} \mathrm{~cm} / \mathrm{s}$. . . . . . . . . 70

4.4 Valores do campo magnético $\mathrm{B}(\mathrm{G})$ como função do tempo (anos) e $\mathrm{r}(\mathrm{kpc})$ para os valores de referência (veja fig. 4.2) . . . . . . . . . 71

5.1 Gráfico extraído de Sirag (1979), onde temos um diagrama da razão momento magnético $P$ e momento angular $U$. Temos a linha contínua representando a previsão da conjectura $S-B, P=$ $\left(G^{1 / 2} / 2 k^{1 / 2}\right) U$. A linha pontilhada representa a regressão linear para os dados dos objetos. . . . . . . . . . . . . . . . . . . 80

5.2 Distribuição de verossimilhança do parâmetro $\beta$ para os dados de AXPs e SGRs. . . . . . . . . . . . . . 86 
5.3 Distribuição de verossimilhança do parâmetro $\theta$ para os dados de AXPs e SGRs. . . . . . . . . . . . . . . . 87

6.1 Para A2050 e A1689, nós mostramos os perfis de massa determinados sem a consideração da pressão não térmica $P_{\mathrm{NT}}$ (curva verde) comparado com os perfis, incluindo as pressões turbulenta e magnética. Estas três curvas representam os perfis de massa, $\alpha=0.5$ (curva azul), $\alpha=0.7$ (curva rosa) e $\alpha=0.9$ (curva amarela), considerando campos magnéticos centrais de $5 \mu \mathrm{G} \ldots \ldots \ldots \ldots$

6.2 Para A2050 e A1689, nós mostramos os perfis de massa determinados sem a consideração da pressão não térmica $P_{\mathrm{NT}}$ (curva verde) comparado com os perfis, incluindo as pressões turbulenta e magnética. Estas três curvas representam os perfis de massa, $\alpha=0.5$ (curva azul), $\alpha=0.7$ (curva rosa) e $\alpha=0.9$ (curva amarela), considerando campos magnéticos centrais de $10 \mu$ G. . . . . . . . . . . . . . . . . 105

6.3 Para A2050 e A1689, nós mostramos os perfis de massa determinados sem a consideração da pressão não térmica $P_{\mathrm{NT}}$ (curva verde) comparado com os perfis, incluindo as pressões turbulenta e magnética. Estas três curvas representam os perfis de massa, $\alpha=0.5$ (curva azul), $\alpha=0.7$ (curva rosa) e $\alpha=0.9$ (curva amarela), considerando campos magnéticos centrais de $30 \mu$ G. . . . . . . . . . . . . . . . 106

6.4 Gráficos de A2050, representando os aglomerados NCC e A1689, representando os aglomerados CC. Mostramos o gráfico da variação da massa em função do valor central do campo magnético $B_{0}$, e do parâmetro de

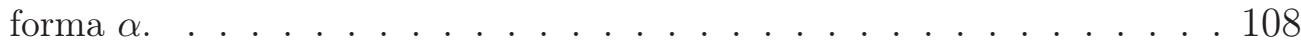


6.5 Gráficos de A2050, representando os aglomerados NCC. No gráfico superior apresentamos a projeção da variação da massa como função de $\alpha$ para vários valores de $B_{0}$ e no gráfico inferior mostramos a projeção da variação da massa como função do campo magnético central $B_{0}$ para

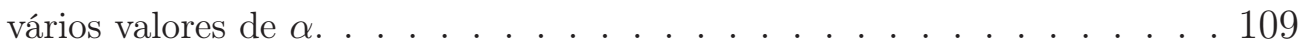

6.6 Gráficos de A1689, representando os aglomerados CC. No gráfico superior apresentamos a projeção da variação da massa como função de $\alpha$ para vários valores de $B_{0}$ e no gráfico inferior mostramos a projeção da variação da massa como função do campo magnético central $B_{0}$ para vários valores

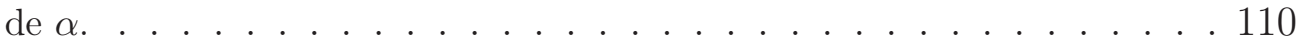




\section{Lista de Tabelas}

3.1 Tamanho e intensidade das bolhas de campo magnético. . . . . . . 51

3.2 Média do campo magnético na linha de visada. . . . . . . . . . . . 51

5.1 Tabela extraída de Sirag (1979), onde nós temos os dados da razão máxima e mínima do momento angular e magnético para corpos celestes. . . . . . . . . . . . . . . . . . . 79

5.2 Soft Gamma-ray Repeaters. Col(1): Nome dos SGRs; Col(2): Período dos SGRs; Col(3): Spindown dos SGRs. . . . . . . . . . 88

5.3 Anomalous X-ray pulsars. $\mathrm{Col}(1)$ : Nome dos AXPs; $\mathrm{Col}(2)$ : Período dos AXPs; $\operatorname{Col}(3)$ : Spindown dos AXPs. . . . . . . . . 88

6.1 Propriedades gerais dos aglomerados. Col (1): nome do aglomerado; Col (2): Ascensão reta; Col (3): Declinação; Col (4): Desvio para o vermelho; Col (5): Raio do aglomerado. . . . . . . . . . . . . . 96 
6.2 Determinação da massa. Col (1): nome do aglomerado; $\operatorname{Col}(2)$ : Modelo de perfil de densidade do gás (Laganá et al. 2008); Col.(3), (4) e (5): Determinação da massa incluindo pressão não térmica, assumindo $B_{0}=10 \mu \mathrm{G}$ e $\alpha=0.5,0.7,0.9$, respectivamente. Col.(6), (7) and (8): Determinação da massa, incluindo pressão não térmica assumindo $B_{0}=30 \mu G$ e $\alpha=0.5,0.7,0.9$, respectivamente. . . . . . 104 


\section{Sumário}

1 Introdução 3

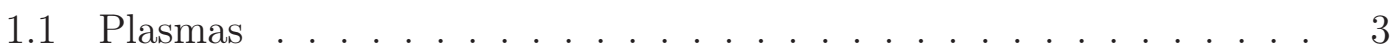

1.2 Campos Magnéticos Primordiais . . . . . . . . . . . . . 6

1.3 Estrutura da Tese . . . . . . . . . . . . . . . . . . . 8

2 Campos Magnéticos Astrofísicos $\quad 11$

2.1 Métodos Observacionais ................. 11

2.1.1 Emissão Síncrotron . . . . . . . . . . . . . . . . 11

2.1.2 Efeito Zeeman . . . . . . . . . . . . . . . 12

2.1.3 Medidas de Rotação Faraday . . . . . . . . . . . . . . . . 12

2.1.4 Medidas de Polarização . . . . . . . . . . . . . . 13

2.2 Campos Magnéticos em Ambientes Astrofísicos . . . . . . . . . . . 14

2.2.1 Estrelas de Baixa Massa . . . . . . . . . . . . . . . . . . 14

2.2.2 Estrelas Massivas . . . . . . . . . . . . . . . 14

2.2.3 Magnetares ................... 15

2.2.4 Discos de Acresção . . . . . . . . . . . . . . . . . . . . . . . 15

2.2.5 Gamma Ray Bursts . . . . . . . . . . . . . . 16

2.2.6 Galáxias . . . . . . . . . . . . . . . . . 16 
2.2.7 Aglomerados de Galáxias . . . . . . . . . . . . . . 16

2.2.8 Vínculos com a radiação cósmica de fundo . . . . . . . . . . 17

2.2.9 Vínculos com Nucleossíntese Primordial . . . . . . . . . 18

2.3 Candidatos à Campos Magnéticos Primordiais . . . . . . . . . . . . 18

2.3.1 Mecanismos Astrofísicos . . . . . . . . . . . . . . 18

2.3.2 Mecanismos Oriundo de Transições de Fase no Universo Primordial .................... . . . 20

3 Criação de Campos Magnéticos no Universo Primordial 23

3.1 Funções de Correlação . . . . . . . . . . . . . . . . . . . . . . 23

3.2 Teorema da Flutuação Dissipação . . . . . . . . . . . . . . . . . . . 25

3.3 Espectro de Freqüência dos Campos Magnéticos . . . . . . . . . . . 30

3.4 Evolução das Flutuações Primordiais . . . . . . . . . . . . . . 33

3.5 Considerações . . . . . . . . . . . . . . . . . . . . . 40

4 Origem dos Campos Magnéticos Galácticos 53

4.1 Dínamo $\alpha-\Omega$.................... . . 54

4.2 Turbulência em Pequena Escala . . . . . . . . . . . . . . . . . 59

4.3 Turbulência Protogaláctica . . . . . . . . . . . . . . 63

4.4 Considerações . . . . . . . . . . . . . . . . . . . . . . . 72

5 Origem de Campos Magnéticos em Objetos Compactos 75

5.1 Conjectura Schuster-Blackett . . . . . . . . . . . . . 76

5.2 Magnetares ....................... 81

5.3 Gamma Ray Bursts . . . . . . . . . . . . . . . . . . . . . 89

5.4 Considerações . . . . . . . . . . . . . . . . . . . . . . . . . . 92 
6 Efeitos de Campos Magnéticos e Turbulência em Aglomerados de $\begin{array}{ll}\text { Galáxias } & 93\end{array}$

6.1 Dados da Amostra . . . . . . . . . . . . . . . . 95

6.2 O perfil do Campo Magnético . . . . . . . . . . . . . . . . . 97

6.3 Turbulência em Aglomerados de Galáxias . . . . . . . . . . . . . . . 99

6.4 Determinação da Massa Incluindo Efeitos das Pressões não Térmicas 101

6.5 Resultados . . . . . . . . . . . . . . . . . 102

6.6 Considerações . . . . . . . . . . . . . . . . . . . 111

7 Conclusões e Perspectivas 113

A Dedução do Teorema da Flutuação-Dissipação 117

B Evolução do Tensor de Correlação das Flutuações Magnéticas 123

$\begin{array}{ll}\text { C Modelo Cosmológico Padrão } & 129\end{array}$

C.1 Métrica de Friedman-Robertson-Walker . . . . . . . . . . . . . . . 129

C.2 Tensor Energia-Momento . . . . . . . . . . . . . . . . . 130

C.3 Equações de Einstein . . . . . . . . . . . . . . . . . . . . . . . . . . 131 
SUMÁRIO 


\section{Capítulo 1}

\section{Introdução}

\subsection{Plasmas}

A maioria dos eventos astrofísicos envolve plasmas. Sendo de extrema importância uma análise de suas propriedades para um melhor entendimento destes fenômenos.

Umas das características mais interessantes do plasma, é sua habilidade de manter-se em um estado de neutralidade de carga. A primeira vista, poderíamos pensar que o campo Coulombiano de qualquer partícula deveria se propagar por todo volume do plasma, contudo este não é o caso. Debye foi o primeiro a apontar que o campo gerado por algum desequilíbrio de carga é blindado, possuindo um alcance restrito (Boyd \& Sanderson 2003). Podemos entender melhor este efeito considerando um íon de carga Ze, em um dado ponto $\mathrm{P}$ de um plasma neutro. $\mathrm{O}$ efeito gerado será uma atração de elétrons para este ponto P enquanto os íons serão repelidos. Logo, o íon será circundado por uma nuvem neutralizadora. Devido sua massa ser muito maior que a do elétron, podemos desprezar os movimentos 
dos íons. Assumindo que a densidade numérica da nuvem eletrônica $n_{c}$ é dada pela distribuição de Boltzmann, $n_{c}=n_{e} \exp \left(e \phi / k_{B} T_{e}\right)$, onde $e, T_{e}$ e $n_{e}$ são a carga, temperatura e densidade do elétron respectivamente e $k_{B}$ é a constante de Bolztmann. Devemos resolver a equação de Poisson para o potencial eletrostático $\phi(r)$ no plasma.

Uma vez que $\phi(r) \rightarrow 0$ se $r \rightarrow \infty$, podemos expandir $\exp \left(e \phi / k_{B} T_{e}\right)$. Pela condição de neutralidade temos que $Z n_{i}=n_{e}$. A equação de Poisson para $r \gg 1$ em torno do ponto $\mathrm{P}$ assumindo simetria esférica torna-se:

$$
\frac{1}{r^{2}} \frac{d}{d r}\left(r^{2} \frac{d \phi}{d r}\right)=\frac{n_{e} e^{2}}{\epsilon_{0} k_{B} T_{e}} \phi
$$

onde $\epsilon_{0}$ é a constante de permissividade do vácuo e $\lambda_{D}$ é o comprimento de Debye definido na equação (1.3). Resolvendo a equação (1.1), para o potencial $\phi(r)$, temos

$$
\phi(r)=\frac{Z e}{4 \pi \epsilon_{0} r} \exp \left(-r / \lambda_{D}\right)
$$

onde

$$
\lambda_{D}=\left(\frac{\epsilon_{0} k_{B} T_{e}}{n_{e} e^{2}}\right)^{1 / 2} \simeq 7.43 \times 10^{3}\left(\frac{T_{e}(e V)}{n_{e}}\right)^{1 / 2} m .
$$

Em regiões maiores que a esfera de Debye (esfera formada em torno do ponto P com raio $\lambda_{D}$ ), o plasma permanece efetivamente neutro. Um requisito necessário pra existência do plasma é que as dimensões do sistema sejam grandes comparadas 
com $\lambda_{D}$. O número de elétrons $N_{D}$ dentro da esfera de Debye é dado por

$$
N_{D}=\frac{4}{3} \pi \lambda_{D}^{3} n_{e}
$$

O inverso deste número é proporcional à razão entre a energia potencial e a energia cinética no plasma, dada por $g=1 / n_{e} \lambda_{D}^{3}$, onde a condição $g \ll 1$ é denominada aproximação de plasma.

Outro parâmetro fundamental dos efeitos coletivos são as oscilações de plasma, que ocorrem em resposta a algum desequilíbrio de carga. O forte campo eletrostático sob o qual os elétrons estão submetidos, causam oscilações em torno da posição de equilíbrio com uma freqüência característica, denominada freqüência de plasma $\omega_{p}$. Esta freqüência é dada por

$$
\omega_{p e}=\frac{\left(k_{B} T_{e} / m_{e}\right)^{1 / 2}}{\lambda_{D}} \simeq 56.4 n_{e}^{1 / 2} s^{-1}
$$

onde $m_{e}$ é a massa do elétron. Qualquer campo aplicado com uma freqüência menor que a freqüência de plasma é impedido de penetrar no plasma devido à rápida resposta dos elétrons, neutralizando o campo. A freqüência correspondente para os íons é dada por

$$
\omega_{p i} \simeq 1.32 Z\left(\frac{n_{i}}{A}\right)^{1 / 2}
$$

onde Z é carga, A, o número atômico e $n_{i}$ é a densidade numérica de íons. 


\subsection{Campos Magnéticos Primordiais}

A origem dos campos magnéticos cósmicos é um dos mais fascinantes problemas da astrofísica moderna, (e.g., Rees 1987; Kronberg 1994). Campos magnéticos são detectados em uma grande variedade de escalas astrofísicas, desde estrelas até super-aglomerados de galáxias. Observações indicam que os campos magnéticos galácticos e extragalácticos possuem intensidades de $\sim \mu \mathrm{G}$ com escalas de coerência de vários kpc (Beck 2008). Tais campos são observados em galáxias tanto em baixo quanto em alto desvio para o vermelho $(z)$.

Um dos modelos inicialmente propostos para explicar a origem destes campos foi o mecanismo de dínamo $\alpha-\Omega$, onde novos campos são gerados continuamente pela ação combinada da rotação diferencial $(\Omega)$ e turbulência helicoidal $(\alpha)$. Entretanto, este mecanismo necessita de um campo magnético semente pré-existente e de um tempo relativamente longo para amplificá-lo.

Existem muitos candidatos para explicar a origem destes campos sementes. Uma das abordagens mais populares é a geração através do mecanismo de Biermann (Biermann 1950). Este mecanismo foi investigado em diversos sistemas astrofísicos, tais como formação de estruturas em grande escala (Peebles 1967; Rees \& Rheinhardt 1972; Wasserman 1978), frentes de ionização cosmológicas, (e.g., Gnedin, Ferrara \& Zweibel 2000), remanescentes de supernovas devido às primeiras estrelas, (e.g., Miranda, Opher \& Opher 1998), etc.

Outra possibilidade é uma origem primordial para estes campos sementes, antes da formação das primeiras galáxias. Por exemplo, campos magnético poderiam ter sido criados durante a transição de fase quark hádron (TFQH), quando o Universo possuía uma temperatura $T_{T F Q H} \cong 1.5 \times 10^{12} \mathrm{~K}$, na transição de fase eletrofraca, 
ou na era inflacionária.

Uma das maiores dificuldades com a maioria destes cenários para criação de campos magnéticos no universo primordial ( $\ll 1 s)$, é seu pequeno comprimento de coerência em $z \lesssim 10$. O comprimento de coerência é limitado pelo raio do horizonte no instante de criação do campo magnético. Portanto, estes campos não teriam um comprimento comóvel suficiente para explicar campos magnéticos em escalas da ordem do tamanho de galáxias.

Neste trabalho, nós sugerimos que os campos magnéticos observados têm sua origem em flutuações eletromagnéticas no plasma existente no universo primordial. Sendo esta, uma forma natural para criar estes campos magnéticos. O Teorema da Flutuação-Dissipação (TFD), prediz grandes flutuações de campos magnéticos no plasma em equilíbrio térmico imediatamente após a TFQH. Tajima et al. (1992) sugeriram que grandes flutuações previstas pelo TFD em épocas remotas não dissiparam, mas continuaram a existir até a época presente e agora contribuem para o campo magnético dominante. Nós evoluímos estes campos magnéticos sementes até $z \sim 10$, utilizando o modelo cosmológico padrão (veja apêndice C). Encontramos campos magnéticos da ordem de $10 \mu G$, contudo com pequenos comprimentos de coerência $\sim 0.1 p c$, nesta época.

De forma a explicar as observações de campos magnéticos em escala galáctica, analisamos a importância da turbulência em pequena escala na amplificação do comprimento de correlação destes campos magnéticos, assim como sua intensidade.

Estudamos a turbulência em regiões protogalácticas, que podem ser geradas por ondas de choque provenientes de instabilidades gravitacionais. Este mecanismo é capaz de aumentar o comprimento de coerência dos nossos campos sementes até os valores de $\mu \mathrm{G}$ observados, em uma escala de tempo de $\sim 10^{8}$ anos. 
Além da origem e evolução destes campos magnéticos primordiais, estudamos a influência de tais campos na determinação da massa de aglomerados de galáxias. Incluímos os termos de pressão magnética e de turbulência na equação de equilíbrio hidrostático e estudamos a variação na massa estimada para 5 aglomerados de Abell. Neste estudo, utilizamos dados em raios-X do XMM-Newton ${ }^{1}$, analisados por Laganá et al. (2008).

Campos magnéticos são importantes não apenas em escalas galácticas, mas também em diversos processos astrofísicos presentes em objetos compactos. Consideramos a possibilidade de explicar a origem dos campos magnéticos existentes em magnetares e possivelmente em gama rays bursts, assumindo um acoplamento entre o momento angular de um objeto e seu campo magnético. Campos magnéticos de alta intensidade são muito difíceis de serem explicados astrofisicamente. Assumindo a validade deste acoplamento, podemos explicar naturalmente sua existência como conseqüência direta do alto momento angular destes objetos.

\subsection{Estrutura da Tese}

No capítulo 2, fizemos uma revisão sobre os campos magnéticos astrofísicos e comentamos as diversas técnicas de observação destes campos. Comentamos os valores observados destes campos magnéticos em diversas escalas, assim como seus vínculos observacionais. Finalizamos com uma discussão sobre os diversos candidatos para origem desses campos.

No capítulo 3, discutimos nosso modelo para origem dos campos magnéticos cosmológicos baseado no TFD. Fizemos uma revisão sobre funções de correlação

\footnotetext{
${ }^{1}$ http://xmm.esac.esa.int/xsa/
} 
e sobre a teoria subjacente ao TFD, discutimos nosso modelo pra evolução destas flutuações desde o universo primordial até hoje.

No capítulo 4, consideramos a possibilidade destes campos sementes serem amplificados na região protogaláctica através do mecanismo de turbulência em pequena escala. Fizemos uma breve revisão sobre a teoria de dínamo $\alpha-\Omega$. Introduzimos a teoria de dínamo em pequena escala e mostramos como ela poderia amplificar nossos campos sementes até os valores observados em galáxias.

No capítulo 5, analisamos a possibilidade dos campos magnéticos em magnetares e gamma ray bursts serem originados devido a um acoplamento entre o momento angular de um objeto e seu momento magnético, sugerida pela conjectura Schuster-Blacket (S-B). Esta conjectura propõe que os campos magnéticos de objetos celestes poderiam ter sua semente originada apenas devido à sua rotação.

No capítulo 6, discutimos os efeitos dos campos magnéticos e turbulência no suporte gravitacional de aglomerados de galáxias. Comparamos a determinação da massa destes aglomerados via raios-X, com e sem a inclusão destes efeitos.

No capítulo 7, apresentamos nossas conclusões e perspectivas. Nos apêndice A deduzimos o Teorema da Flutuação Dissipação aplicado em plasmas. No apêndice B, fizemos uma breve discussão da dedução das equações para evolução da correlação dos campos magnéticos devido à turbulência em pequena escala. Além do corpo da tese, colocamos em anexo os artigos resultantes de nosso trabalho, tanto os publicados, submetido quanto em fase final de redação. 
CAPÍTULO 1. INTRODUÇÃO 


\section{Capítulo 2}

\section{Campos Magnéticos Astrofísicos}

\subsection{Métodos Observacionais}

\subsubsection{Emissão Síncrotron}

A emissão síncrotron é a radiação produzida por elétrons relativísticos espiralando ao longo das linhas de campo magnético. É usada para inferência de campos magnéticos em ambientes astrofísicos desde pulsares até aglomerados de galáxias. Assumindo uma distribuição de energia dos elétrons da forma

$$
n_{e}(E) d E=n_{e 0}\left(\frac{E}{E_{0}}\right)^{-\gamma} d E
$$

onde o expoente $\gamma$ é denominado índice espectral, $E$ é a energia do elétron, $n_{e}$ é a densidade eletrônica e $\nu$ é a freqüência da emissão. O índice 0 representa um valor de referência. Podemos escrever a emissividade síncrotron $j_{\nu}$ para uma dada fonte da forma 


$$
j_{\nu} \propto n_{e 0} \nu^{(1-\gamma)^{2} / 2} B_{\perp}^{(1+\gamma) / 2} .
$$

O espectro de emissão síncrotron pode então ser relacionado com a distribuição de elétrons e a intensidade do campo magnético perpendicular a linha de visada $\left(B_{\perp}\right)$.

\subsubsection{Efeito Zeeman}

No vácuo, os níveis de energia do elétron em um átomo são independentes da direção do seu momento angular. A presença de campos magnéticos quebra esta degenerescência, definindo uma direção preferencial no espaço, gerando $2 j+1$ níveis de energia, onde $j$ é o número quântico associado ao momento angular total $(J)$ do átomo. A diferença de energia entre estes níveis vizinhos é dada por $\Delta E=g \mu B$ (Rybicki \& Lightman 1979), onde $g$ é o fator de Landé, $\mu$ é o magnéton de Bohr e $B$ o campo magnético. O efeito Zeeman é uma forma direta de detectar um campo magnético astrofísico. Se medirmos $\Delta E$ através da análise do espectro do objeto, inferimos $B$ sem maiores hipóteses adicionais. Uma das linhas espectrais mais usadas no cálculo de campos magnéticos por efeito Zeeman em galáxias é a linha de $21 \mathrm{~cm}$ do hidrogênio neutro (Widrow 2002).

\subsubsection{Medidas de Rotação Faraday}

Ondas eletromagnéticas ao passarem em um meio permeado por campos magnéticos e elétrons livres, sofrem uma rotação nos seus estados de polarização circular esquerdo e direito. Estes estados viajam com velocidades de fase diferentes neste meio. Logo, uma onda linearmente polarizada sofrerá uma rotação de seu 
plano de polarização dada por

$$
\Delta \theta=\frac{e^{3} \lambda^{2}}{2 \pi m_{e}^{2} c^{4}} \int_{0}^{L} n_{e}(l) B_{\|}(l) d l,
$$

onde $m_{e}$ é a massa do elétron, $\lambda$ é o comprimento de onda da radiação, $\Delta \theta$ o ângulo de rotação do plano de polarização, $n_{e}$ é a densidade de elétrons ao longo da linha de visada, L é a distância percorrida pela radiação e $B_{\|}$, é a componente do campo magnético ao longo da linha de visada. Medidas de rotação Faraday são muito utilizadas para determinação dos campos magnéticos astrofísicos, particularmente em galáxias e aglomerados de galáxias.

\subsubsection{Medidas de Polarização}

A análise da polarização da luz estelar é útil no estudo dos campos magnéticos em nossa galáxia e sua vizinhança. Os campos magnéticos galácticos podem ser observados no óptico através da polarização da luz estelar pelos grãos de poeira do meio. Estes grãos podem ser alongados e se alinhar de forma perpendicular às linhas de campo magnético. Como conseqüência, a radiação transmitida tem uma direção de polarização paralela à direção do campo magnético do meio. Observações de um grande número de estrelas dão informação complementar aos outros métodos sobre o aspecto geral do campo magnético na galáxia. 


\subsection{Campos Magnéticos em Ambientes Astrofísi- $\cos$}

\subsubsection{Estrelas de Baixa Massa}

Tanto no Sol quanto em outras estrelas de baixa massa, observamos campos da ordem de kG através de medidas do efeito Zeeman e outras características espectrais relacionadas ao campo magnético. Espera-se que estes campos magnéticos sejam mantidos pelo mecanismo de dínamo gerado pela rotação diferencial. Contudo, ainda não entendemos perfeitamente o processo de rotação diferencial, a origem do ciclo solar, o papel da rotação diferencial no dínamo em grande escala entre outras características (Uzdensky 2009).

\subsubsection{Estrelas Massivas}

Observações indicam a existência de campos magnéticos de $\sim 1 \mathrm{kG}$ em estrelas do tipo $\mathrm{O}$ e $\mathrm{B}$, com massas da ordem de $10-50 M_{\odot}$. Isto pode ser muito importante no entendimento das propriedades destas estrelas massivas, assim como na sua evolução. Contudo, a origem destes campos magnéticos em estrelas massivas ainda é pouco compreendida. Visto que elas não possuem envelopes convectivos onde o dínamo possa operar, espera-se que a origem dos seus campos seja devido a processos diferentes daqueles presentes em estrelas de baixa massa (Uzdensky 2009). 


\subsubsection{Magnetares}

Magnetares são uma classe de estrelas de nêutrons altamente magnetizadas $\left(B \gtrsim 10^{14} G\right)$, o argumento mais convincente, apesar de indireto, para estes altos campos vem da sua alta taxa de spindown. O campo magnético destas estrelas de nêutrons, assumindo que sua desaceleração ocorre devido à emissão de radiação de dipolo magnético no vácuo, é de $\sim B_{d i p}=2.48 \times 10^{14}(P / 6 s)^{1 / 2}\left(\dot{P} / 10^{11} s s^{-1}\right)^{1 / 2} G$. O qual, para estes objetos, é três ordens de grandeza maior que o campo magnético encontrado em rádio pulsares. O modelo de magnetar foi introduzido Duncan \& Thompson (1992). Os intensos campos magnéticos dos magnetares não são fáceis de ser produzidos, sendo ainda um desafio à compreensão do mecanismo progenitor.

\subsubsection{Discos de Acresção}

Discos gasosos em rotação em torno de um objeto central são geralmente encontrados em diversos ambientes astrofísicos. Por exemplo em estrelas jovens, objetos compactos estelares (anãs brancas, estrelas de nêutrons e buracos negros), ou mesmo em buracos negros supermassivos existentes nos centros galácticos. Observações do efeito Zeeman em linhas de masers provenientes do disco de acresção da galáxia Seyfert II NGC 4258, impoem limites superiores $(B<50 m G)$, para a componente toroidal do campo magnético a uma distância de 0.2 pc do buraco negro central (Modjaz et al. 2003). Mapas de rotação Faraday em regiões de quasares e rádio galáxias com jatos relativísticos, também revelam que o meio em escalas ao redor de núcleos ativos de galáxias precisa ser significativamente magnetizado (Zavala \& Taylor 2003). 


\subsubsection{Gamma Ray Bursts}

Alguns autores consideram a possibilidade de que campos magnéticos intensos ( $B \sim 10^{15} G$ ), sejam necessários para explicar a energia gerada por gamma ray burts (GRBs), (Piran 2005; Lee et al. 2000; Lei et al. 2005, 2008). A fonte seria o buraco negro central, cuja extração de energia poderia ocorrer devido ao mecanismo de Blandford-Znajek (Blandford \& Znajek 1977). Campos desta intensidade seriam capazes de extrair energia do buraco negro em escalas de tempo de até $\sim 1000 \mathrm{~s}$, podendo assim explicar os surtos de raios- $\gamma$.

\subsubsection{Galáxias}

A intensidade dos campos magnéticos em galáxias pode ser determinada através do espectro síncrotron, assumindo equipartição entre a densidade de energia magnética e a dos raios cósmicos. O valor médio típico do campo magnético, sob esta hipótese, para galáxias espirais é da ordem de $10 \mu \mathrm{G}$, coerentes em escalas da ordem de dezenas de kpc (Beck 2008). Campos mais intensos, de 50-100 $\mu \mathrm{G}$, são encontrados em galáxias com surtos de formação estelar. Também foram detectados campos de $84 \mu \mathrm{G}$, através do efeito Zeeman, em uma galáxia com desvio para o vermelho $z=0.692$ (Walsh et al. 2002). Campos Magnéticos com intensidade similar são observados tanto em galáxias espirais quanto em elípticas e irregulares (Widrow 2002).

\subsubsection{Aglomerados de Galáxias}

Campos magnéticos em aglomerados de galáxias podem ser inferidos através de medidas de rotação Faraday, tanto de rádio galáxias quanto de fontes em rádio 
observadas através do aglomerado. Observamos campos magnéticos de $\mu \mathrm{G}$ com comprimentos de coerência de vários kpc nos aglomerados de galáxias. Técnicas observacionais utilizando rotação Faraday, assumindo isotropia na estrutura destes campos magnéticos, estimam valores de 3G em Abell 2634, 6G em Abell 400 e 12G em Hydra A. Seus comprimentos de coerência avaliados foram de 4.9, 3.6 e 0.9 kpc, respectivamente. Usando um simples modelo, onde o meio intraglomerado consiste de células de campo magnético com tamanho e intensidade uniformes, mas com distribuição aleatória, Clarke, Kronberg, \& Böhringer (2001) estimaram campos magnéticos da ordem de $5(l / 10 k p c)^{-1 / 2} \mathrm{G}$ nos aglomerados, onde $l$ é o comprimento de coerência do campo magnético.

\subsubsection{Vínculos com a radiação cósmica de fundo}

Campos magnéticos primordiais geram rotação Faraday na polarização linear da radiação cósmica de fundo. Esta polarização é gerada pelo espalhamento Compton inverso na superfície de último espalhamento. Os dados de 5 anos do Wilkinson Microwave Anisotropy Probe (WMAP) colocam um limite superior para magnitude da polarização do modo B. Estes resultados impõe um limite na amplitude comóvel dos campos magnéticos primordiais na faixa de 0.4 a $30 \mathrm{nG}$ sobre uma distância comóvel de $1 \mathrm{Mpc}$, dependendo do espectro de potência do campo magnético. Em uma escala de $100 \mathrm{Mpc}$ o campo médio precisa ser menor que $0.7 \mathrm{nG}$ para qualquer espectro de potência (Kahniashvili et al. 2008). 


\subsubsection{Vínculos com Nucleossíntese Primordial}

A existência de campos magnéticos nos estágios iniciais do Universo tem efeitos tanto na sua taxa de expansão, quando na estatística dos processos de decaimento $\beta$, principalmente através da modificação da distribuição estatística dos elétrons e pósitrons. Vínculos oriundos da nucleossíntese primordial restringem estes campos magnéticos para valores de $B \leqslant 10^{12} \mathrm{G}$, com comprimentos de coerência $10 \ll L \ll$ $10^{11} \mathrm{~cm}$ comóveis (Grasso \& Rubinstein 1996).

\subsection{Candidatos à Campos Magnéticos Primordiais}

\subsubsection{Mecanismos Astrofísicos}

\section{Bateria de Biermann}

O termo de bateria de Biermann surge na equação de indução magnética, quando temos gradientes não paralelos de densidade e pressão $(\nabla \rho \times \nabla p \neq 0)$. Gnedin, Ferrara \& Zweibel (2000) investigaram a geração de campos magnéticos devido este mecanismo em frentes de ionização cosmológicas, usando simulações da reionização, ocasionadas por estrelas, em protogaláxias. Eles consideraram dois mecanismos: 1) a fuga da frente de ionização das protogaláxias; e 2) a propagação das frentes de ionização através da filamentos neutros de alta densidade. O primeiro mecanismo ocorre antes da sobreposição das regiões ionizadas $(z \approx 7)$, enquanto o segundo mecanismo continua a funcionar após esta época. Em sua simulação, eles acharam campos médios de $\approx 10^{-18} \mathrm{G}$, para objetos com massa $10^{9}-10^{10} M_{\odot}{ }^{1}$. Subramanian et al. (1994) sugeriram que o mecanismo de Bier-

\footnotetext{
${ }^{1} M_{\odot}=$ Massa solar
} 
mann poderia ocorrer em frentes de ionização se propagando através de flutuações

de densidade. Eles estimaram campos de $10^{-20} \mathrm{G}$, com comprimento de coerência de vários kpc.

\section{Criação de Campos Magnéticos por Explosões de Supernovas Primor- diais}

Explosões primordiais de supernovas poderiam ser a origem dos campos magnéticos no universo (Miranda \& Opher 1996, 1997; Miranda, Opher \& Opher 1998). Neste cenário, Miranda, Opher \& Opher (1998) acharam campos de $4 \times 10^{-10} \mathrm{G}$, com comprimentos de coerência da ordem de 100 kpc. Entretanto, seu mecanismo supõe que a maior parte do fluxo magnético seja formada por objetos de massa $\sim 10^{6} M_{\odot}$, em $z \approx 300$, em desacordo com modelos cosmológicos de formação dos primeiros objetos, onde as primeiras estrelas teriam se formado por volta de $z=$ 65, (Naoz et al. 2006).

\section{Origem de Campos Magnéticos por Jatos Extragalácticos}

Daly \& Loeb (1990) e Jafelice \& Opher (1992) sugeriram que a magnetização do meio intergaláctico poderia ser resultado da corrente elétrica carregada pelos jatos extragalácticos. Estes jatos seriam gerados por núcleos ativos de galáxias em alto z. Campos magnéticos gerados por estas correntes seriam da ordem de $\sim$ $10^{-8} \mathrm{G}$ sobre regiões comóveis de alguns Mpc.

\section{Campos Magnéticos Gerados por Perturbações Cosmológicas}

Takahashi et al. (2005) estudaram perturbações cosmológicas, considerando a evolução do plasma com 3 componentes: elétrons, prótons, e fótons. Eles anali- 
saram os termos de colisão entre elétrons e fótons e consideraram a possibilidade dos mesmos poderem induzir campos magnéticos da ordem de $10^{-19} \mathrm{G}$ sobre uma escala comóvel de $10 \mathrm{Mpc}$ na época do desacoplamento da matéria com radiação.

\subsubsection{Mecanismos Oriundo de Transições de Fase no Uni- verso Primordial}

\section{Campos Magnéticos Gerados na Transição de Fase Quark-Hádron}

Um possível cenário de geração de campos magnéticos foi proposto por Quashnock et al. (1989). Campos elétricos seriam criados atrás das frentes de choque devido a expansão de bolhas geradas pela transição de fase quark-hádron. Como conseqüência das diferentes equações de estado para as componentes bariônicas e leptônicas, um forte gradiente de pressão seria produzido pela passagem da onda de choque, dando origem ao campo elétrico radial atrás da onda. Quashnock et al. (1989) estimaram campos elétricos com intensidade de

$$
e E \simeq 15\left(\frac{\epsilon}{0.1}\right)\left(\frac{\delta}{0.1}\right)\left(\frac{k T_{Q H P T}}{150 M e V}\right)\left(\frac{100 \mathrm{~cm}}{l}\right) \frac{k e V}{c m}
$$

onde $\epsilon$ é a razão de densidade de energia entre os dois fluidos, $\delta \equiv(l \Delta p / p), \Delta$ p é o gradiente de pressão e $l$ é a distância média comóvel entre as regiões de nucleação das bolhas. Eles sugeriram que campos magnéticos seriam produzidos nas regiões de colisão entre estas frentes de choque, dando origem à turbulência e vorticidade da ordem de $l$. Eles encontraram campos magnéticos de tamanho comóvel de $\sim 1$ $\mathrm{UA}^{2}$ com uma intensidade atual de $\sim 2 \times 10^{-17}$ G. Seguindo a mesma idéia de

\footnotetext{
${ }^{2} 1 \mathrm{UA}=1.49598 \times 10^{13} \mathrm{~cm}$
} 
campos magnéticos gerados na época da TFQH, Cheng \& Olinto (1994) estimaram campos magnéticos de $\sim 10^{-16} \mathrm{G}$ na época atual, com comprimentos de coerência de $\sim 1$ pc. Em escalas galácticas, estes campos teriam o valor atual de $\simeq 10^{-20} \mathrm{G}$.

\section{Campos Magnéticos Gerados na Transição de Fase Eletrofraca}

A transição de fase eletrofraca poderia gerar campos magnéticos primordiais (Baym et al. 1996; Sigl et al. 1997). Durante esta fase, ocorre a quebra de simetria de calibre do grupo eletrofraco $S U(2)_{L} \times U(1)_{Y}$ para o grupo eletromagnético $U(1)_{E M}$. Teríamos o plasma com um resfriamento rápido abaixo da temperatura eletrofraca, $\simeq 100 \mathrm{GeV}$. As bolhas geradas por esta quebra de simetria iriam nuclear e expandir preenchendo o Universo. O típico tamanho comóvel do raio de Hubble neste época era $L_{H} \approx 10 \mathrm{~cm}$ com temperaturas $T_{H} \approx 100 \mathrm{GeV}$. O tamanho comóvel das bolhas seria de $L_{B}=f_{B} L_{H}$, que seriam criadas com $f_{B} \simeq 10^{-3}-10^{-2}$ (Baym et al. 1996). O campo magnético gerado nesta fase seria da ordem de

$$
B \simeq(4 \pi \epsilon)^{1 / 2}\left(T_{E W}\right) T_{E W}^{2}\left(\frac{v_{\text {wall }}}{c}\right)^{2} \simeq\left(7 \times 10^{21}-2 \times 10^{24}\right) G
$$

onde $\epsilon=g_{*} a T_{E W}^{4} / 2 \simeq 4 \times 10^{11} \mathrm{GeV} \mathrm{fm}^{-3}$ é a densidade de energia na época da transição eletrofraca (Widrow 2002).

\section{Campos Magnéticos Gerados Durante a Inflação}

A inflação pode naturalmente produzir efeitos em escalas muito maiores que o horizonte de Hubble (Turner \& Widrow 1988). Se flutuações eletromagnéticas fossem amplificadas durante a inflação, apareceriam hoje como campos magnéticos com grande escala de coerência. Várias maneiras de quebrar a invariância conforme 
e gerar estes campos foram propostas. Turner \& Widrow (1988) consideraram três possibilidades:

1. Introduzindo um acoplamento da forma $R A_{\mu} A^{\mu}$ ou $R_{\mu \nu} A^{\mu} A^{\mu}$, onde $\mathrm{R}$ é o escalar de Ricci, $R_{\mu \nu}$ o tensor de Ricci, e $A^{\mu}$ é o campo eletromagnético. Estes termos quebram a invariância de calibre e dão aos fótons uma dependência efetiva da massa com o tempo. Turner \& Widrow (1988) mostraram que para uma escolha razoável de parâmetros, tal mecanismo poderia gerar os campos magnéticos galácticos, sem a necessidade do dínamo galáctico;

2. introduzindo termos da forma $R_{\mu \nu \lambda \kappa} F^{\mu \nu} F^{\lambda \kappa} / m^{2}$ ou $R F^{\mu \nu} F_{\mu \nu}$, onde m é algum escalar de massa e F o tensor eletromagnético. Este mecanismo pode dar conta apenas de um campo primordial muito pequeno;

3. acoplando o fóton à algum campo carregado, que quebra a invariância conforme. 


\section{Capítulo 3}

\section{Criação de Campos Magnéticos no Universo Primordial}

\subsection{Funções de Correlação}

Qualquer quantidade física caracterizando um sistema macroscópico em equilíbrio pode sofrer desvios em seu valor médio. Estes desvios são denominados flutuações desta quantidade física e dependem da temperatura e outras propriedades macroscópicas do sistema. As flutuações do nosso sistema podem ser descritas pelas funções de correlação. Estas funções definem os valores médios dos produtos das flutuações de uma ou várias quantidades do nosso sistema em diferentes pontos do espaço e do tempo. Esta média é feita sobre todos os estados quânticos do sistema e sobre toda as distribuições estatísticas destes estados. Se o meio for espacialmente homogêneo podemos definir estas flutuações definindo um vetor $\langle\mathbf{j}(\mathbf{r}, t)\rangle=0$, distribuído continuamente no espaço. A função de correlação das 
componentes $j_{i}$ e $j_{j}$ do vetor $\mathbf{j}$ será

$$
\left\langle j_{i}\left(r_{1}, t_{1}\right) j_{j}\left(r_{2}, t_{2}\right)\right\rangle \equiv\left\langle j_{i} j_{j}\right\rangle_{\mathbf{r} t}
$$

onde $\mathbf{r}=r_{2}-r_{1}$ e $t=t_{2}-t_{1}$. As componentes de Fourier de $\left\langle j_{i} j_{j}\right\rangle_{\mathbf{r} t}$ podem ser assim escritas,

$$
\begin{array}{r}
\left\langle j_{i} j_{j}\right\rangle_{\mathbf{k} \omega}=\int d t \int d \mathbf{r} e^{-i \mathbf{k} \cdot \mathbf{r}+i \omega t}\left\langle j_{i} j_{j}\right\rangle_{\mathbf{r} t} \\
\left\langle j_{i} j_{j}\right\rangle_{\mathbf{r} t}=\frac{1}{(2 \pi)^{4}} \int d \omega \int d \mathbf{k} e^{i \mathbf{k} \cdot \mathbf{r}-i \omega t}\left\langle j_{i} j_{j}\right\rangle_{\mathbf{k} \omega}
\end{array}
$$

onde $\left\langle j_{i} j_{j}\right\rangle_{\mathbf{k} \omega}$ é a distribuição espectral da função de correlação. Podemos relacionála com a média do produto das componentes de Fourier,

$$
\left\langle j_{i}^{\dagger}(\mathbf{k}, \omega) j_{j}\left(\mathbf{k}^{\prime}, \omega^{\prime}\right)\right\rangle=(2 \pi)^{4}\left\langle j_{i} j_{j}\right\rangle_{\mathbf{k} \omega} \delta\left(\mathbf{k}-\mathbf{k}^{\prime}\right) \delta\left(\omega-\omega^{\prime}\right),
$$

onde o índice $\dagger$ denota o conjugado hermitiano ${ }^{1}$.

Podemos definir a função de correlação espacial, ao avaliar a função de correlação em um mesmo instante de tempo,

$$
\left\langle j_{i}\left(r_{1}, t\right) j_{j}\left(r_{2}, t\right)\right\rangle \equiv\left\langle j_{i} j_{j}\right\rangle_{\mathbf{r}}
$$

A componente de Fourier desta função de correlação espacial é dada pela integral em todas as freqüências,

\footnotetext{
${ }^{1} \mathrm{~A}$ aplicação $\dagger$ em um dado operador, representa a composição das operações conjugação complexa e transposição da matriz representada por este operador.
} 


$$
\left\langle j_{i} j_{j}\right\rangle_{\mathbf{k}}=\frac{1}{2 \pi} \int d \omega\left\langle j_{i} j_{j}\right\rangle_{\mathbf{k} \omega}
$$

De forma análoga podemos definir a função de correlação temporal como a média avaliada em uma dada região do espaço em diferentes instantes de tempo,

$$
\left\langle j_{i}\left(\mathbf{r}, t_{1}\right) j_{j}\left(\mathbf{r}, t_{2}\right)\right\rangle \equiv\left\langle j_{i} j_{j}\right\rangle_{t}
$$

cuja componente de Fourier é dada por

$$
\left\langle j_{i} j_{j}\right\rangle_{\omega}=\frac{1}{(2 \pi)^{3}} \int d \mathbf{k}\left\langle j_{i} j_{j}\right\rangle_{\mathbf{k} \omega} .
$$

\subsection{Teorema da Flutuação Dissipação}

O teorema da Flutuação-Dissipação relaciona a distribuição espectral das flutuações com as propriedades dissipativas do meio (dedução completa no apêndice A).

$$
\left\langle j_{i} j_{j}\right\rangle_{\mathbf{k} \omega}=\frac{\hbar}{e^{\hbar \omega / k_{B} T}-1} i\left[\alpha_{i j}^{*}(\omega, \mathbf{k})-\alpha_{i j}(\omega, \mathbf{k})\right],
$$

onde $\alpha_{i j}$ é denominado tensor resposta, que define a reação do sistema às flutuações em torno do equilíbrio.

Em um plasma isotrópico, a função resposta $\alpha_{i j}$ pode ser escrita como,

$$
\alpha_{i j}(\omega, \mathbf{k})=\frac{k_{i} k_{j}}{k^{2}} \alpha_{l}(\omega, \mathbf{k})+\left(\delta_{i j}-\frac{k_{i} k_{j}}{k^{2}}\right) \alpha_{t}(\omega, \mathbf{k}) .
$$

Os coeficientes transversais $\alpha_{t}$ e longitudinais $\alpha_{l}$ relacionam-se com as permis- 
sividades dielétricas transversal $\epsilon_{t}$ e longitudinal $\epsilon_{l}$ do plasma,

$$
\begin{array}{r}
\alpha_{t}(\omega, \mathbf{k})=\frac{\omega^{2}}{4 \pi}\left(1-\zeta^{2}\right) \frac{\epsilon_{t}(\omega, k)-1}{\epsilon_{t}(\omega, k)-\zeta^{2}} \\
\alpha_{l}(\omega, \mathbf{k})=\frac{\omega^{2}}{4 \pi} \frac{\epsilon_{l}(\omega, k)-1}{\epsilon_{l}(\omega, k)}
\end{array}
$$

onde $\zeta=k c / \omega$.

Se tivermos isotropia, as flutuações longitudinais e transversais são mutuamente independentes. As componentes da corrente transversal e longitudinal, são relacionadas aos campos elétricos através da relações

$$
\begin{array}{r}
j_{l}(\omega, \mathbf{k})=\frac{i \omega}{4 \pi} \mathbf{E}_{l}(\omega, \mathbf{k}), \\
j_{t}(\omega, \mathbf{k})=\frac{i \omega}{4 \pi}\left(1-\zeta^{2}\right) \mathbf{E}_{t}(\omega, \mathbf{k}) .
\end{array}
$$

A distribuição espectral das flutuações de corrente pode ser obtida substituindo as equações (3.11) e (3.12) na equação (3.9),

$$
\begin{aligned}
\left\langle j_{i} j_{j}\right\rangle_{\mathbf{k} \omega}= & \frac{1}{2 \pi} \frac{\hbar \omega^{2}}{e^{\hbar \omega / k_{B} T}-1} \times \\
& {\left[\frac{k_{i} k_{j}}{k^{2}} \frac{I m \epsilon_{l}}{\left|\epsilon_{l}\right|^{2}}+\left(\delta_{i j}-\frac{k_{i} k_{j}}{k^{2}}\right)\left(1-\frac{k^{2} c^{2}}{\omega^{2}}\right)^{2} \frac{I m \epsilon_{t}}{\left|\epsilon_{t}-\frac{k^{2} c^{2}}{\omega^{2}}\right|^{2}}\right] . }
\end{aligned}
$$

O primeiro e segundo termos descrevem as flutuações das correntes longitudinais e transversais respectivamente. Usando a equação da continuidade, $\partial \rho / \partial t+\nabla \cdot j=$ 0, podemos relacionar as flutuações de densidade de carga com as flutuações de corrente longitudinais. No caso isotrópico temos

$$
\left\langle\rho^{2}\right\rangle_{\mathbf{k} \omega}=\frac{k^{2}}{2 \pi} \frac{\hbar}{e^{\hbar \omega / k_{B} T}-1} \frac{I m \epsilon_{l}}{\left|\epsilon_{l}\right|^{2}} .
$$


Usando a equação (3.14), obtemos a seguinte expressão para a distribuição espectral da intensidade de flutuações do campo elétrico (Kubo 1957; Akhiezer et al. 1975; Sitenko 1967; Rostoker et al. 1965; Dawson 1968):

$$
\begin{array}{r}
\frac{1}{8}\left\langle E_{i} E_{j}\right\rangle_{k \omega}=\frac{i}{2} \frac{\hbar}{e^{\hbar \omega / k_{B} T}-1}\left(\Lambda_{i j}^{-1}-\Lambda_{i j}^{-1 *}\right), \\
\Lambda_{i j}(\omega, \mathbf{k})=\frac{k^{2} c^{2}}{\omega^{2}}\left(\frac{k_{i} k_{j}}{k^{2}}-\delta_{i j}\right)+\epsilon_{i j}(\omega, \mathbf{k}), \\
\epsilon_{i j}(\omega, \mathbf{k})=\frac{k_{i j}}{k^{2}} \epsilon_{l}(\omega, \mathbf{b})+\left(\delta_{i j}-\frac{k_{i} k_{j}}{k^{2}} \epsilon_{t}(\omega, \mathbf{k})\right),
\end{array}
$$

onde $\epsilon_{i j}(\omega, \mathbf{k})$ é o tensor dielétrico do plasma. Da lei da Faraday temos $\mathbf{B}=c \mathbf{k} / \omega \times$ $\mathbf{E}$ e junto com $\mathbf{k}=k \hat{\mathbf{x}}$, encontramos as flutuações magnéticas perpendiculares $B_{2}$ e $B_{3}$

$$
\frac{\left\langle B_{2}^{2}\right\rangle_{k \omega}}{8 \pi}=\frac{i}{2} \frac{\hbar}{e^{\hbar \omega / k_{B} T}-1} \frac{c^{2} k^{2}}{\omega^{2}}\left(\Lambda_{33}^{-1}-\Lambda_{33}^{-1 *}\right)
$$

e

$$
\frac{\left\langle B_{3}^{2}\right\rangle_{k \omega}}{8 \pi}=\frac{i}{2} \frac{\hbar}{e^{\hbar \omega / k_{B} T}-1} \frac{c^{2} k^{2}}{\omega^{2}}\left(\Lambda_{22}^{-1}-\Lambda_{22}^{-1 *}\right),
$$

onde os subscritos 1, 2 e 3 referem-se a x, y e z. As flutuações totais do campo magnético são

$$
\frac{\left\langle B^{2}\right\rangle_{k \omega}}{8 \pi}=\frac{i}{2} \frac{\hbar}{e^{\hbar \omega / k_{B} T}-1} \frac{c^{2} k^{2}}{\omega^{2}}\left(\Lambda_{22}^{-1}+\Lambda_{33}^{-1}-\Lambda_{22}^{-1 *}-\Lambda_{33}^{-1 *}\right) .
$$

Com o intuito de obter $\Lambda_{i j}(\omega, \mathbf{k})$ das equações de movimento do plasma, utilizamos um modelo de multifluidos para o plasma,

$$
m_{\alpha} \frac{d \mathbf{v}_{\alpha}}{d t}=e_{\alpha} \mathbf{E}-\eta_{\alpha} m_{\alpha} \mathbf{v}_{\alpha}
$$


onde $\alpha$ é a espécie da partícula e $\eta_{\alpha}$ é a freqüência de colisão das espécies $\alpha$. A transformada de Fourier da equação (3.23) é dada por

$$
-i \omega m_{\alpha} v_{\alpha}=e_{\alpha} E-\eta_{\alpha} m_{\alpha} v_{\alpha},
$$

o qual gera uma corrente $j_{\alpha}$,

$$
j_{\alpha}=\frac{\omega_{p \alpha}^{2}}{4 \pi\left(-i \omega+\eta_{\alpha}\right)} E .
$$

Podemos escrever a corrente $j_{\alpha}$ em termos do tensor de susceptibilidade magnética $\chi_{\alpha i j}$ e do campo elétrico $E$, tal que

$$
j_{\alpha i}=-i \omega \chi_{\alpha i j}(\omega \mathbf{k}) E_{j}(\omega \mathbf{k}) .
$$

O tensor dielétrico é dado por

$$
\begin{aligned}
\epsilon_{i j}(\omega \mathbf{k}) & =\delta_{i j}+4 \pi \sum_{\alpha} \chi_{\alpha i j}, \\
4 \pi \chi_{\alpha i j}^{\omega \mathbf{k}} & =\frac{\omega_{p \alpha}^{2}}{\omega\left(\omega+i \eta_{\alpha}\right)} \delta_{i j} .
\end{aligned}
$$

Podemos assim, escrever o tensor dielétrico da forma

$$
\epsilon_{i j}(\omega, \mathbf{k})=\delta_{i j}-\sum_{\alpha} \frac{\omega_{p \alpha}^{2}}{\omega\left(\omega+i \eta_{\alpha}\right)} \delta_{i j}
$$

onde $\omega_{p \alpha}$ é a freqüência de plasma de uma dada espécie $\alpha$. Vamos considerar um plasma de elétrons e pósitrons, pois estamos interessados em avaliar estas flutuações no Universo primordial quando a temperatura era $\mathrm{T} \sim 1 \mathrm{MeV}$. Neste 
plasma de elétrons e pósitrons a frequiência de plasma dos elétrons é igual a dos pósitrons, $\omega_{p e^{+}}=\omega_{p e^{-}}$e $\eta_{e^{+}}=\eta_{e^{-}}=\eta$. A equação (3.28) torna-se então

$$
\epsilon_{i j}(\omega, \mathbf{k})=\delta_{i j}-\frac{\omega_{p}^{2}}{\omega(\omega+i \eta)} \delta_{i j}
$$

onde $\omega_{p}^{2}=\omega_{p e^{+}}^{2}+\omega_{p e^{-}}^{2}$. Temos que $\eta_{e}$ é a freqüência de colisão de Coulomb, para elétrons $\eta_{e}=2.91 \times 10^{-6} n_{e} \ln \Lambda T^{-3 / 2}(\mathrm{eV}) \mathrm{s}^{-1}$, onde $n_{e}$ é a densidade de elétrons e $\ln \Lambda=\ln 4 \pi n_{e} \lambda_{D}^{3}$ é o logaritmo de Coulomb. Para o caso de um plasma de elétrons e prótons após a nucleossíntese primordial, $\eta_{p}=4.78 \times 10^{-18} n_{e} T^{-3 / 2} \ln \Lambda(\mathrm{eV}) \mathrm{s}^{-1}$. Obtemos então

$$
\Lambda_{i j}=\left(\begin{array}{ccc}
1-\frac{\omega_{p}^{2}}{\omega(\omega+i \eta)} & 0 & 0 \\
0 & 1-\frac{c^{2} k^{2}}{\omega^{2}}-\frac{\omega_{p}^{2}}{\omega(\omega+i \eta)} & 0 \\
0 & 0 & 1-\frac{c^{2} k^{2}}{\omega^{2}}-\frac{\omega_{p}^{2}}{\omega(\omega+i \eta)}
\end{array}\right)
$$

Podemos escrever as flutuações de campo magnético no espaço de freqüência $\omega$ e número de onda $k$, como função da temperatura

$$
\begin{aligned}
\frac{\left\langle B^{2}\right\rangle_{k \omega}}{8 \pi}= & \frac{2 \hbar \omega}{e^{\hbar \omega / k_{B} T}-1} \eta \omega_{p}^{2} \times \\
& \frac{k^{2} c^{2}}{\left(\omega^{2}+\eta^{2}\right) k^{4} c^{4}+2 \omega^{2}\left(\omega_{p}^{2}-\omega^{2}-\eta^{2}\right) k^{2} c^{2}+\left[\left(\omega^{2}-\omega_{p}^{2}\right)^{2}+\eta^{2} \omega^{2}\right] \omega^{2}},
\end{aligned}
$$

(Tajima et al. 1992; de Souza \& Opher 2008). Para um plasma primordial onde efeitos relativísticos são importantes fazemos a associação $\omega_{p} \rightarrow \omega_{p} / \sqrt{\gamma}$, onde $\gamma=1 / \sqrt{1-v^{2} / c^{2}}$ é o fator de Lorentz. 


\subsection{Espectro de Freqüência dos Campos Magnéti-}

$\cos$

A equação (3.31) tem um limite bem conhecido, se fizermos $\eta \rightarrow 0$ e $\omega_{p} \rightarrow 0$ (um limite onde não há plasma), chegamos na equação para um corpo negro no vácuo

$$
\frac{\left\langle B^{2}\right\rangle_{\omega}}{8 \pi}=\int d k \frac{\left\langle B^{2}\right\rangle_{k \omega}}{8 \pi}=\frac{1}{2} \frac{\pi \hbar}{e^{\hbar \omega / k_{B} T}-1} \frac{\omega^{3}}{c^{3}}
$$

ou seja, a presença do plasma modifica as flutuações magnéticas. Desejamos inicialmente encontrar o espectro de flutuações em função da frequência $\left\langle B^{2}\right\rangle_{\omega}$. Integrando a equação (3.31), obtemos

$$
\frac{\left\langle B^{2}\right\rangle_{\omega}}{8 \pi}=\frac{1}{\pi^{2}} \frac{\hbar \omega}{e^{\hbar \omega / k_{B} T}-1} \frac{2 \eta}{\omega_{p e}^{2}}\left(\frac{\omega_{p e}}{c}\right)^{3} \int_{0}^{\infty} d x \frac{x^{4}}{\left(\omega^{\prime 2}+\eta^{\prime 2}\right) x^{4}+\ldots},
$$

onde $x=k c / \omega_{p e}, \omega^{\prime}=\omega / \omega_{p e}$ e $\eta^{\prime}=\eta / \omega_{p e}$. Note entretanto, que esta equação diverge para altos números de onda. Esta divergência ocorre, pois a análise feita é baseada em equações clássicas, e para números de onda muito grandes (ou pequenos comprimentos de onda) os efeitos das colisionais tornam-se importantes e a descrição de fluido torna-se falha.

Considere uma onda eletromagnética se propagando no plasma. A relação de dispersão das ondas é fortemente dependente dos efeitos coletivos do plasma. Ondas de menor comprimento de onda são menos afetadas pelo plasma. Se ela tiver um comprimento de onda muito menor que a escala de colisão $\sim c / \omega_{p}$, irá viajar pelo plasma como se estivesse viajando pelo espaço vazio. Como vimos anteriormente, o espectro para freqüências muito maiores que $\omega_{p}$, tende ao espectro 
de corpo negro. Então podemos considerar o limite de alto número de onda fazendo $\eta \rightarrow 0$, obtendo desta forma

$$
\frac{\left\langle B^{2}\right\rangle_{\mathbf{k} \omega}}{8 \pi}=\frac{2 \hbar \omega}{e^{\hbar \omega / k_{B} T}-1} \omega_{p}^{2} k^{2} c^{2} \pi \delta\left[\frac{\omega\left(\omega^{2}-c^{2} k^{2}-\omega_{p}^{2}\right)}{\omega^{2}-c^{2} k^{2}}\right] \frac{1}{\left(\omega^{2}-c^{2} k^{2}\right)^{2}} .
$$

Integrando a equação (3.34) sobre $4 \pi k^{2} d k$ de 0 até $\infty$, obtemos

$$
\frac{\left\langle B^{2}\right\rangle_{\omega}}{8 \pi}=\frac{k_{B} T}{2 \pi} \delta(\omega) \int \frac{\omega_{p}^{2}}{\omega_{p}^{2}+c^{2} k^{2}} k^{2} d k+\frac{1}{2 \pi c^{3}} \frac{\hbar}{e^{\hbar \omega / k_{B} T}-1}\left(\omega^{2}-\omega_{p}^{2}\right)^{3 / 2} .
$$

Podemos dividir a equação (3.33) em duas partes, uma de 0 até $k_{c u t}\left(x_{c u t}=\right.$ $\left.k_{c u t} c / \omega_{p e}\right)$, e outra de $k_{c u t}$ até $\infty$, onde na primeira parte mantemos $\eta$, pois os efeitos colisionais são importantes para baixas freqüências como discutimos anteriormente. Para a segunda integral podemos tomar $\eta \rightarrow 0$, visto que os efeitos colisionais são desprezíveis. Obtemos assim

$$
\begin{array}{r}
\frac{\left\langle B^{2}\right\rangle_{\omega}}{8 \pi}=\frac{1}{\pi^{2}} \frac{\hbar \omega^{\prime}}{e^{\left(\hbar \omega_{p e} / k_{B} T\right) \omega^{\prime}}-1} 2 \eta^{\prime}\left(\frac{\omega_{p e}}{c}\right)^{3} \int_{0}^{x_{c u t}} d x \frac{x^{4}}{\left(\omega^{\prime 2}+\eta^{\prime 2}\right) x^{4}+\ldots} \\
+\frac{\hbar\left(\omega^{\prime 2}-\omega_{p}^{\prime 2}\right)^{3 / 2}}{2 \pi\left[e^{\hbar \omega_{p e} / k_{B} T}-1\right]}\left(\frac{\omega_{p e}}{c}\right)^{3} \Theta\left(\omega-\sqrt{c^{2} k_{c u t}^{2}+\omega_{p}^{2}}\right),
\end{array}
$$

onde $\Theta$ é a função degrau de Heaviside ${ }^{2}$. Note que a divergência para $\omega \rightarrow 0$ foi removida,

$$
\lim _{\omega \rightarrow 0} \frac{\left\langle B^{2}\right\rangle_{\omega}}{8 \pi}=\frac{\hbar \omega^{\prime}}{\pi^{2}\left(e^{\hbar \omega_{p e} \omega^{\prime} / k_{B} T}-1\right)} 2\left(\frac{\omega_{p e}}{c}\right)^{3} \frac{1}{\eta^{\prime}} \int_{0}^{x_{c u t}} d x=\frac{2 k_{B} T}{\pi^{2} \eta^{\prime} \omega_{p e}}\left(\frac{\omega_{p e}}{c}\right)^{3} x_{c u t} .
$$

A escolha de $x_{c u t}$ é feita de forma a termos uma junção suave entre o espectro no

$$
{ }^{2} \Theta(x)=\left\{\begin{array}{lll}
0 & \text { se } & x<0 \\
\frac{1}{2} & \text { se } & x=0 \\
1 & \text { se } & x>0
\end{array}\right.
$$


limite de baixas freqüências e o espectro de corpo negro para altas freqüências. Teremos então que $k_{c u t} \sim \omega_{p e} / c\left(x_{c u t} \sim 1\right)$.

Da mesma forma, integrando a equação (3.31) sobre $\omega$, teremos o espectro em $k$,

$$
\frac{\left\langle B^{2}\right\rangle_{k}}{8 \pi}=\frac{k_{B} T}{2}\left[\frac{1}{1+k^{2} c^{2} / \omega_{p}^{2}}+\frac{\hbar}{e^{\hbar\left(\omega_{p}^{2}+k^{2} c^{2}\right)^{1 / 2}-1}} \frac{k^{2} c^{2}}{\left(\omega_{p}^{2}+k^{2} c^{2}\right)^{1 / 2}}\right],
$$

o primeiro termo da equação (3.38), surge apenas na presença de um plasma sendo mais importante no regime de pequenos números de onda $\mathrm{k}$. O segundo termo corresponde a radiação de corpo negro modificada pela presença do plasma. No limite de $\hbar \rightarrow 0$, temos a lei da equipartição,

$$
\frac{\left\langle B^{2}\right\rangle_{k}}{8 \pi}=\frac{k_{B} T}{2}
$$

O espectro de freqüência próximo de $\omega=0$, (onde os efeitos de plasma são relevantes) é dado por

$$
\frac{\left\langle B^{2}\right\rangle_{\omega=0}}{8 \pi}=\frac{3}{2 \pi^{3}} \sqrt{\frac{3}{\pi}} k_{B} T\left(\frac{\omega_{p}}{c}\right)^{3} .
$$

Isto significa que as flutuações magnéticas são proporcionais à temperatura e a densidade $\left(n_{e}^{3 / 2}\right)$ do plasma. Estas flutuações não são importantes em plasmas atuais, devido à baixa temperatura e densidade, contudo no Universo primordial podem ser bastante significativas. 


\subsection{Evolução das Flutuações Primordiais}

Como vimos anteriormente, o plasma primordial pode ter flutuações magnéticas significativas. Estas flutuações, também chamadas bolhas magnéticas, surgem e decaem espontaneamente a todo instante. Estas pequenas bolhas podem entretanto coalescer em bolhas maiores em um processo de auto-organização.

Podemos analisar este processo de ponto de vista de uma equação de Bolztmann para um espaço em expansão,

$$
\frac{\partial n_{i}}{\partial t}=-\frac{n_{i}}{\tau i^{2}}+3 H n_{i}+n_{i-1}\left\langle n_{1} v \sigma\right\rangle,
$$

onde consideramos a formação de uma estrutura composta por $i$ bolhas magnéticas, sendo $n_{i}$ a densidade de bolhas em uma estrutura de ordem $i$. Na equação acima, $\tau$ é o tempo de vida da bolha, $\sigma$ sua seção de choque, $v$ a sua velocidade média da bolha e $H$ é o parâmetro de Hubble. Como estamos interessados em analisar a evolução destas flutuações, ao longo da expansão do Universo, todas as quantidades consideradas são comóveis. Utilizamos o modelo cosmológico padrão $\Lambda C D M$ (mais detalhes no apêndice C) para evoluir nossas equações. Neste caso o parâmetro de Hubble pode ser escrito da forma

$$
H(z)=H_{0} \sqrt{\Omega_{m 0}(1+z)^{3}+\Omega_{\Lambda}+\Omega_{r 0}(1+z)^{4}},
$$

onde $H_{0}=72 \mathrm{kms}^{-1} M p c^{-1}$, é a constante de Hubble, $\Omega_{m}=8 \pi G \rho / 3 H^{2}=0.28$ (parâmetro de densidade da matéria), $\Omega_{\Lambda}=\lambda c^{2} / 3 H^{2}=0.72$ (parâmetro de densidade do vácuo), $\Omega_{r}=32 \pi G \sigma T / 3 H^{2} c^{3}$ (parâmetro de densidade da radiação). O índice 0 refere-se as quantidades medidas hoje, $z=0$, (Weinberg 2008). 
Consideramos flutuações de campo magnético criadas no plasma primordial imediatamente após a transição de fase quark-hádron. Consideramos um plasma de elétrons, pósitrons e fótons após a TFQH.

As flutuações eletromagnéticas em nosso plasma primordial podem ser divididas principalmente em duas categorias: uma com grandes comprimentos de onda $\left(\mathrm{k} \lesssim \omega_{p e} / \mathrm{c}\right)$ e próximas da frequência zero $\left(\omega \ll \omega_{p e}\right)$ e outras com comprimentos de onda bem pequenos $\left(\mathrm{k} \gg \omega_{p e} / \mathrm{c}\right)$ e frequência bem maior que $\omega_{p e}$. Os modos $k \lesssim \omega_{p e} / c$ são significativamente modificados pelo plasma.

Podemos estimar a intensidade dos campos magnéticos para um dado comprimentos de onda $\lambda$,

$$
\left\langle B^{2}\right\rangle_{\lambda} / 8 \pi=\left(k_{B} T / 2\right)(4 \pi / 3) \lambda^{-3}
$$

$\operatorname{para} \lambda=2 \pi c / \omega_{p}$

$$
\left(\left\langle B^{2}\right\rangle_{\lambda_{p}}\right)^{1 / 2}=1.4 \times 10^{-12}\left[n /\left(10^{4} \mathrm{~cm}^{3}\right)\right]^{3 / 4}\left[T /\left(10^{4} K\right)\right]^{1 / 2} G
$$

Intuitivamente podemos interpretar este teorema, imaginando que um certo modo individual do campo, decai por algum efeito dissipativo, aumentando a energia das partículas e excitando outros modos. Este processo se repete gerando uma certa quantidade de flutuações relacionadas à dissipação do meio. Imagine elétrons se propagando no plasma. Eles podem contribuir para as flutuações de corrente devido à sua característica colisional. Flutuações eletromagnéticas serão induzidas por estas flutuações de corrente. Entretanto as componentes de baixa freqüência não se propagam no plasma. Logo, isto irá gerar um amortecimento nas flutuações do campo. O tempo de vida destas flutuações é relacionado ao tempo de colisão 
(ou a algum mecanismo de dissipação cinética) característico do meio.

Podemos escrever a relação de dispersão pra estes modos da forma (Tajima et al. 1992)

$$
\omega^{2}-k^{2} c^{2}-\frac{\omega_{p}^{2}}{1+i \eta / \omega}=0
$$

No limite de baixas freqüências a equação (3.45) torna-se:

$$
\omega=i \eta \frac{k^{2} c^{2}}{\omega_{p}^{2}}
$$

O tamanho espacial $\lambda$ das flutuações de campo magnético é relacionado $\operatorname{com} \tau$, o tempo de vida da flutuação, por

$$
\lambda(\tau)=2 \pi \frac{c}{\omega_{p}}\left(\eta_{e} \tau\right)^{1 / 2}
$$

(Tajima et al. 1992). O tamanho médio destas flutuações é dado por

$$
\bar{\lambda}=\frac{\int \lambda\left[\left\langle B^{2}\right\rangle_{\lambda} / 8 \pi\right] d \lambda}{\int\left[\left\langle B^{2}\right\rangle_{\lambda} / 8 \pi\right] d \lambda}=\frac{7 \pi}{3}\left(c / \omega_{p}\right) .
$$

Usando o modelo de Tajima et al. (1992). Nós assumimos que estas flutuações podem ser descritas por uma bolha de tamanho $\bar{\lambda}$ aproximada por um dipolo magnético com intensidade do campo dada pela equação (3.43).

As bolhas magnéticas estavam na temperatura do plasma e colidiam e coalesciam em um tempo dado por $t_{c o a l}=\bar{\lambda} / v_{b u b}$, onde $v_{b u b}$ é a velocidade térmica da bolha. O tempo de coalescência $t_{\text {coal }}$ foi calculado, mostrando-se menor que o tempo de vida $\tau$ das bolhas no Universo primordial. Ele era de $\sim 10^{-5}$ s instantes após a TFQH em $\mathrm{t} \sim 10^{-4} \mathrm{~s}$ após o big bang. Antes do campo magnético se dissi- 
par, as bolhas podiam coalescer uma com a outra. Bolhas maiores foram formadas com tempo de vida $\tau \propto \lambda^{2}$, bolhas maiores vivem mais e têm maior possibilidade de colidir com outras, ocorrendo preferencialmente a formação de grandes bolhas.

Iniciamos nossos cálculos imediatamente após a TFQH e continuamos até z $\sim 10\left(\sim 10^{9}\right.$ anos após o big bang), quando as galáxias começam a se formar. Estes campos magnéticos foram amplificados adiabaticamente quando a matéria bariônica colapsou formado as galáxias.

Embora a densidade de energia dos dipolos magnéticos vizinhos seja da mesma ordem da densidade de energia do campo magnético médio quando eles não estão a uma distância curta um do outro, a densidade de energia magnética cresce apreciavelmente quando os dipolos vizinhos se aproximam. Como o campo magnético do dipolo é proporcional a $r^{-3}$, onde $r$ é a distância entre eles, a densidade de energia magnética entre os dipolos vizinhos é proporcional a $r^{-6}$. Consequentemente, a densidade de energia magnética das bolhas magnéticas adjacentes com distâncias de separação muito curtas é muito maior que a densidade média de energia magnética.

Os dipolos tendem a se alinhar devido a intensificação da energia de interação magnética a uma curta distância inter-dipolar. A taxa de interação entre os dipolos depende da sua velocidade. Nós usamos como velocidade característica a velocidade da massa da bolha de plasma, a qual está em equilíbrio térmico com a temperatura do Universo em um dado desvio para o vermelho $z$.

Quando os dipolos estão orientados de forma oposta e interagindo, dois processos opostos ocorrem: o alinhamento e a reconexão. Como os dipolos atuam um no outro eles tendem a se alinhar em um tempo de giro $\tau_{f l i p} \sim 10^{-5}$ s instantes após a TFQH em $\mathrm{t} \sim 10^{-4} \mathrm{~s}$, onde $\tau_{\text {flip }}$ é o tempo no qual o dipolo alinha com o 
seu vizinho devido o torque magnético. Nós temos que $\tau_{f l i p} \propto\left(I / N_{\text {mag }}\right)^{1 / 2}$, onde $N_{\text {mag }}$ é o torque magnético e $I$ é o momento de inércia da bolha. Por outro lado, dipolos com campos magnéticos opostos se reconectam em um tearing mode time $\tau_{\text {tear }}$. O menor $\tau_{\text {tear }}$ é de $\bar{\tau}_{\text {tear }} \cong 10^{0.20} \tau_{A}^{1 / 2} \tau_{R}^{1 / 2}$, onde $\tau_{A}=L / v_{A}$ é o tempo de Alfvén e $\tau_{R}=4 \pi L^{2} / c \eta$ o tempo de difusão (Sturrock 1994). O mais curto tearing time instantes após a TFQH é $\sim 10^{15} \mathrm{~s}$. Assim $\tau_{\text {flip }} \ll \bar{\tau}_{\text {tear }}$ pouco após $10^{-4} \mathrm{~s}$ e permanece assim por todas as épocas de interesse. A fig. 3.1 mostra $\tau_{f l i p}$, a fig. 3.2 mostra $\tau_{\text {tear }}$ e a fig. 3.3 sua razão, no intervalo de tempo $\sim 10^{-4}-10^{2} \mathrm{~s}$.

O tempo final usado nas figuras 3.1-3.3 é de $\sim 100$ s. Este é o tempo no qual o campo magnético nas bolhas necessita da idade do Universo para difundir totalmente. A difusão magnética é inversamente proporcional ao quadrado do diâmetro da bolha, sendo relevante apenas em instantes iniciais do nosso cálculo, quando as bolhas eram pequenas. O campo magnético inicial na bolha difunde totalmente em um tempo $\tau_{\text {diff }}=4 \pi \sigma L^{2}$, onde L é o diâmetro da bolha e $\sigma$ é a condutividade elétrica (Grasso \& Rubinstein 2001).

No regime de altas temperaturas $(\mathrm{T}>1 \mathrm{MeV})$ nós seguimos o tratamento de Ahonen \& Enqvist (1996), que resolveram numericamente a equação de Boltzmann para o Universo primitivo. Para $T \lesssim 100 \mathrm{MeV}$ eles encontraram uma condutividade de $\sigma \simeq 0.76 T$. Como imediatamente após a TFQH, a temperatura do Universo era $\sim 100 \mathrm{MeV}$, nós usamos $\sigma \simeq 0.76 T$ para $T>1 \mathrm{MeV}$.

Para temperaturas $T<1 \mathrm{MeV}$ a condutividade pode ser aproximada por

$$
\sigma=\frac{m_{e}}{\alpha \ln \Lambda}\left(\frac{2 T}{\pi m_{e}}\right)^{3 / 2},
$$

onde $\Lambda=\left(1 / 6 \pi^{1 / 2}\right)\left(1 / \alpha^{1 / 2}\right)\left(m_{e}^{3} / n_{e}\right)^{1 / 2}\left(T / m_{e}\right)$, e $\alpha, m_{e}$, e $n_{e}$ é a constante de estru- 
tura fina, a massa do elétron, e a densidade do elétron, respectivamente (Jackson 1975). Para L 1 U.A., $\tau_{\text {diff }}$ é igual a idade do Universo (Grasso \& Rubinstein ). Em nosso modelo as bolhas alcançam o tamanho de $\sim 1$ U.A. em um tempo $\sim 100$ s. Nas figs. 3.1-3.3 $\tau_{\text {flip }}$ e $\tau_{\text {tear }}$ estão traçados desde o instante da TFQH $\left(\sim 10^{-4} s\right)$ até $\sim 100 \mathrm{~s}$.

O campo magnético na bolha deveria dissipar antes dela coalescer se o tempo de difusão magnética fosse menor que o tempo de coalescência. Na fig. 3.4, nós traçamos a razão entre o tempo de coalescência $\tau_{\text {coal }}$ e o tempo de difusão $\tau_{\text {diff }}$. Pode ser visto na fig. 3.4 que a razão permanece muito menor que a unidade em tempos remotos.

Em tempos mais tardios, quando o tempo de giro magnético (i.e., o tempo para que dipolos adjacentes se alinhem) era muito maior que o tempo de Hubble, os dipolos magnéticos permaneceram randômicos. O instante de transição, quando os campos randômicos começam a existir, é $z \sim 10^{8}$. Nesta época, o tamanho comóvel das bolhas era $\sim 1$ pc. De forma a explicar os campos magnéticos galácticos com este modelo, nós precisamos avaliar o campo sobre a escala de uma protogaláxia, $\sim 200 \mathrm{kpc}(\sim 2 M p c$ comóveis em $z=10)$, que sofreu um colapso gravitacional no processo de formação das galáxias até a escalas de $\sim 30 \mathrm{kpc}$.

O campo magnético na bolha diminui adiabaticamente com a expansão do Universo. Uma vez que o fluxo é conservado, nós temos

$$
B=\frac{B_{0}}{a^{2}}
$$

onde $a$ é o fator de escala cósmico. Podemos avaliar o valor de $a$ resolvendo a equação $\dot{a}(t) / a(t)=H(t)$ (Weinberg 2008). 
Nas figs. 3.5 e 3.6, nós mostramos a evolução do tamanho das bolhas como função do tempo, desde imediatamente após a TFQH em $t \sim 10^{-4}$ s até o $z \sim 10$ no instante $t \sim 10^{16} \mathrm{~s}$. Inicialmente, o tamanho das bolhas aumenta rapidamente, como mostrado na fig. 3.5. Neste gráfico, nós observamos o tamanho físico da bolha aumentando desde $10^{-8} \mathrm{~cm} \mathrm{em} t \approx 10^{-4}$ até $1 \mathrm{~cm}$ em um tempo de $10^{-7} \mathrm{~s}$. Ela continua a aumentar nesta taxa até alcançar o tamanho de $\sim 10^{7} \mathrm{~cm}$. A taxa de crescimento então diminui, como mostrado na fig. 3.6. Em $z \sim 10^{8}(t \sim 3000 s)$, o tamanho físico da bolha era $\sim 10^{10} \mathrm{~cm}$ (i.e., um tamanho comóvel $\sim 1 \mathrm{pc}$ ).

Podemos escrever a média do campo magnético em escalas escalas cosmológicas usando a análise utilizada por Grasso \& Rubinstein (2001). O valor médio do campo magnético de tamanho $r$ em um dado instante de tempo $t$ sobre uma região de tamanho $L$ é proporcional a $(r / L)^{p}$, onde podemos ter $\mathrm{p}=1 / 2,1$ ou $3 / 2$,

$$
\langle B(L, t)\rangle_{r m s}=B_{0}\left(\frac{a_{0}}{a(t)}\right)^{2}\left(\frac{r}{L}\right)^{p}
$$

Se nós estamos interessados em uma média volumétrica de uma distribuição randômica de dipolos em uma esfera de diâmetro L, e cada dipolo é uma célula de diâmetro $r$, o campo magnético médio é proporcional a $(r / L)^{3 / 2}$ com $\mathrm{p}=3 / 2$ na equação (3.51) (Widrow 2002). Entretanto, se nós estivermos interessados em um campo magnético médio na linha de visada, sentido por partículas de raios cósmicos ou fótons (i.e. nas medidas de Rotação Faraday) o campo magnético médio é proporcional a $(r / L)^{1 / 2}$, com $\mathrm{p}=1 / 2$ na equação $(3.51)$.

Uma discussão detalhada de procedimentos de média de campos magnéticos foi feita por Hindmarsh \& Everett (1998). O campo magnético nas bolhas como função do tempo é mostrado na fig. 3.7. Na fig. 3.8, a evolução da média volu- 
métrica e na linha de visada sobre uma distância comóvel de $\sim 1$ Mpc é mostrada como função do tempo desde $t \simeq 3 \times 10^{3}$ s até $\mathrm{z} \sim 10\left(\sim 10^{16} s\right)$. A tabela (3.1) mostra o crescimento do campo magnético em nosso modelo e o tamanho das bolhas até $z \sim 10$. O $z$ de equipartição na tabela (3.1) foi obtido da relação $\left(1+z_{e q}\right) \approx 2.3 \times 10^{4} \Omega_{m} h^{2}$ (Padmanabhan 1993). A tabela (3.2) mostra o crescimento do campo magnético médio na linha de visada sobre o tamanho comóvel de uma protogaláxia $\mathrm{L} \sim 1 \mathrm{Mpc}$.

\subsection{Considerações}

Um campo magnético médio não desprezível pode ser importante na formação das primeiras estrelas e na reionização do Universo. A formação dos primeiros objetos marca a transformação do Universo de um estado inicial suave até o estado atual de aglomerações. Em modelos cosmológicos usuais, as primeiras fontes de luz começam a se formar em $z \sim 30$ e reionizaram a maioria do hidrogênio do Universo por volta de $6<z<14$ (Barkana \& Loeb 2001; Cooke et al. 2009). Em geral, se tem achado dificuldades em reionizar o Universo com uma função da massa inicial de Salpeter para as primeiras fontes estelares formadas por flutuações padrão no espectro de matéria escura (Cen 2003; Fukugita \& Kawasaki 2003; Ciardi et al. 2003; Somerville \& Livio 2003; Haiman \& Holder 2003). Campos magnéticos primordiais produzem flutuações adicionais nos bárions pela força de Lorentz (Tashiro \& Sugiyama 2006). A tensão magnética é mais efetiva em escalas pequenas onde o emaranhamento dos campos magnéticos é grande. Tashiro \& Sugiyama concluíram que fótons ionizantes das estrelas de População III formadas em halos escuros poderiam facilmente ter reionizado o Universo por volta de $z$ 
$\simeq 10-20$, considerando que a intensidade do campo magnético atual é $B_{0} \sim \mathrm{nG}$ em uma escala comóvel de $\sim 0.1 \mathrm{Mpc}$. Uma força de Lorentz relevante no colapso da matéria bariônica é proporcional a

$$
\vec{\nabla} \cdot\left[\left(\vec{\nabla} \times \vec{B}_{0}(\vec{x})\right) \times \vec{B}_{0}(\vec{x})\right] \sim \frac{B_{0}^{2}}{L^{2}} \equiv F .
$$

Assim, Tashiro \& Sugiyama (2006) encontraram que o valor de $F \sim 10^{-28} G^{2} / p c^{2}$ é importante na formação dos primeiros objetos. Em nosso modelo a valor atual do campo magnético médio sobre uma escala comóvel $L$ é $B_{0} \sim 0.1 \mu \mathrm{G}(L(p c))^{-3 / 2}$. Nós temos então $F=\left[10^{-14} / L^{2}\right] G^{2} / p c^{2}$. Obtemos o valor de $F$ igual ao de Tashiro \& Sugiyama com $L \sim$ kpc. Nós achamos que uma região comóvel $L \sim \operatorname{kpc}$ em nosso modelo produz uma força de Lorentz que pode ser importante na formação das primeiras fontes estelares e na reionização do Universo. Este comprimento é maior que o comprimento magnético de Jeans. Seus respectivos números de onda, dados por Tashiro \& Sugiyama (2006), são $k_{M J} \sim 32 M p c^{-1} B_{0}^{-1}(n G)$ e $k_{c} \sim$ $102 \mathrm{Mpc}^{-1} B_{0}^{-1}(n G)$. Colocando nossa média volumétrica do campo magnético $B_{0}$ sobre $L \sim 1 \mathrm{kpc}$ nestas expressões, nós obtemos $k_{M J} \sim 10 \mathrm{kpc}^{-1}$ e $k_{c} \sim 34 \mathrm{kpc}^{-1}$. Deve ser notado que uma esfera de diâmetro comóvel $\sim 1$ kpc contém uma massa $\sim 10^{3} M_{\odot}$ para o modelo cosmológico adotado.

Em $z=10$, a intensidade do campo magnético na bolha cujo tamanho comóvel é $\sim 1$ pc era $\sim 9 \mu \mathrm{G}$. Tomando a média na linha de visada sobre uma escala comóvel de $1 \mathrm{Mpc}(\sim 100 \mathrm{kpc}$ em $z \sim 10)$, o campo magnético médio em $z=10$ era $9 \times 10^{-3} \mu \mathrm{G}$. No colapso de uma região comóvel de $1 \mathrm{Mpc}$ em $z=10$ em uma galáxia (tamanho comóvel $\sim 30 \mathrm{kpc}$ ), o campo é amplificado para $\sim 10 \mu \mathrm{G}$. Isto indica que os campos magnéticos criados imediatamente após a TFQH podem ser 
a origem dos campos magnéticos da ordem de $\sim \mu \mathrm{G}$ observados em galáxias em alto e baixo desvio para o vermelho. 


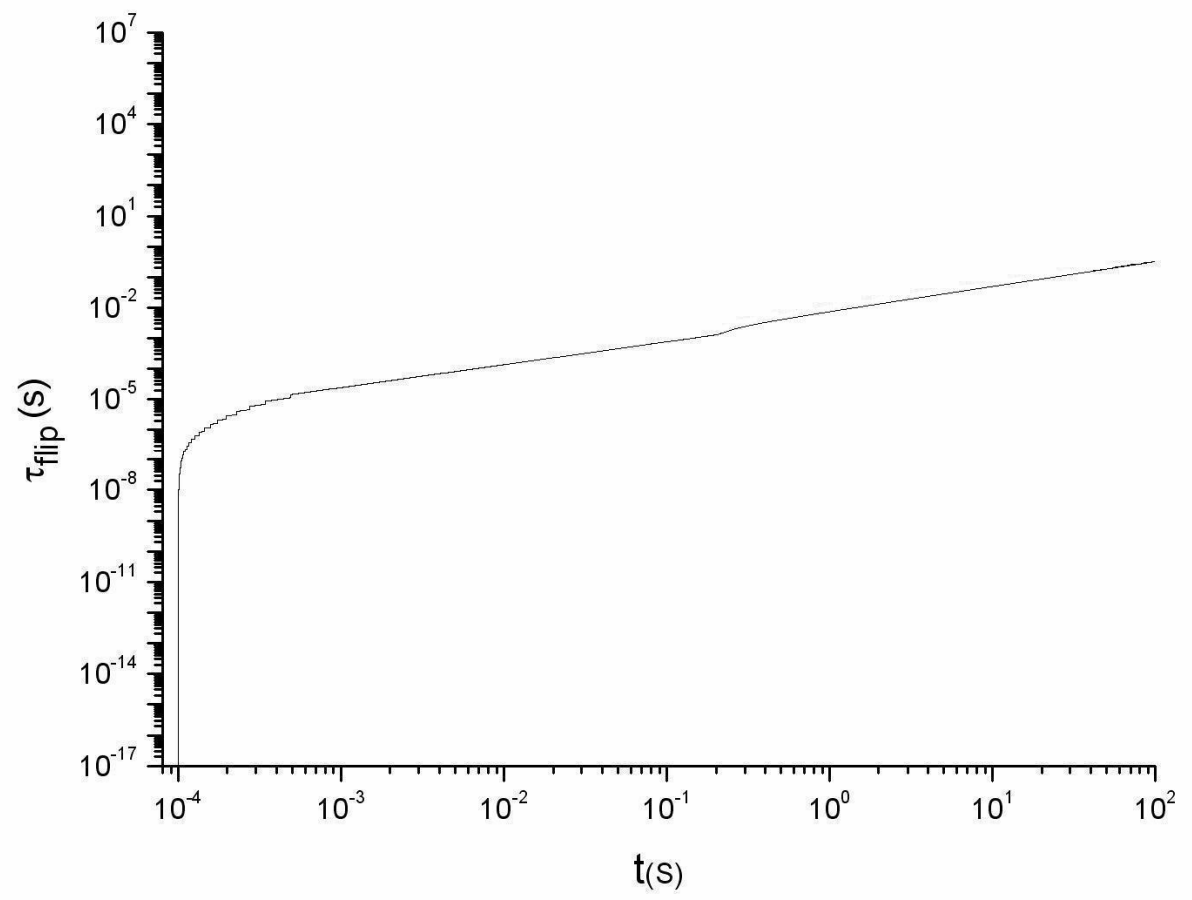

Figura 3.1: Evolução do tempo de giro $\tau_{\text {flip }}(\mathrm{s})$ das bolhas magnéticas em função do tempo t(s). 
CAPÍTULO 3. CRIAÇÃO DE CAMPOS MAGNÉTICOS NO UNIVERSO PRIMORDIAL

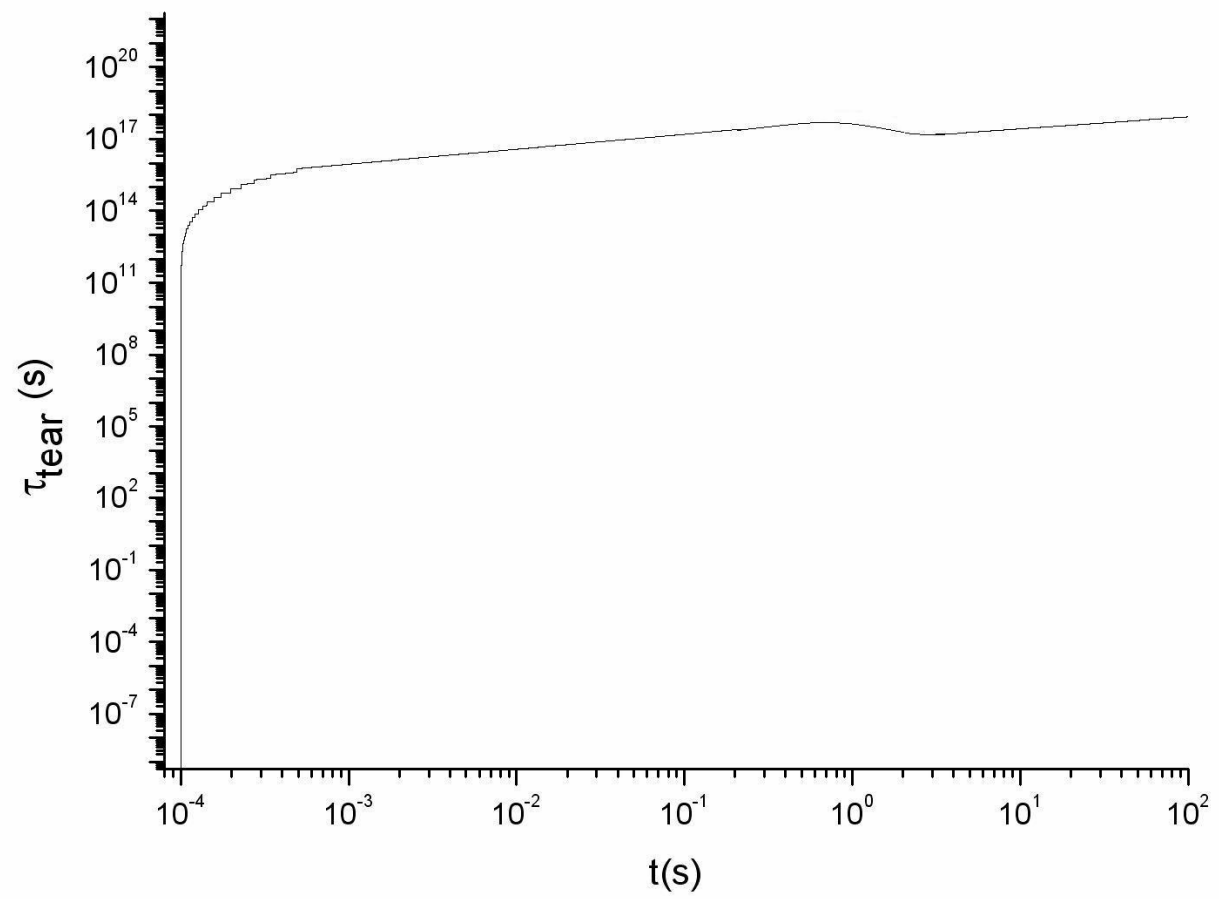

Figura 3.2: Evolução do tempo de reconexão $\tau_{\text {tear }}$ (s) das bolhas magnéticas em função do tempo $t(s)$. 


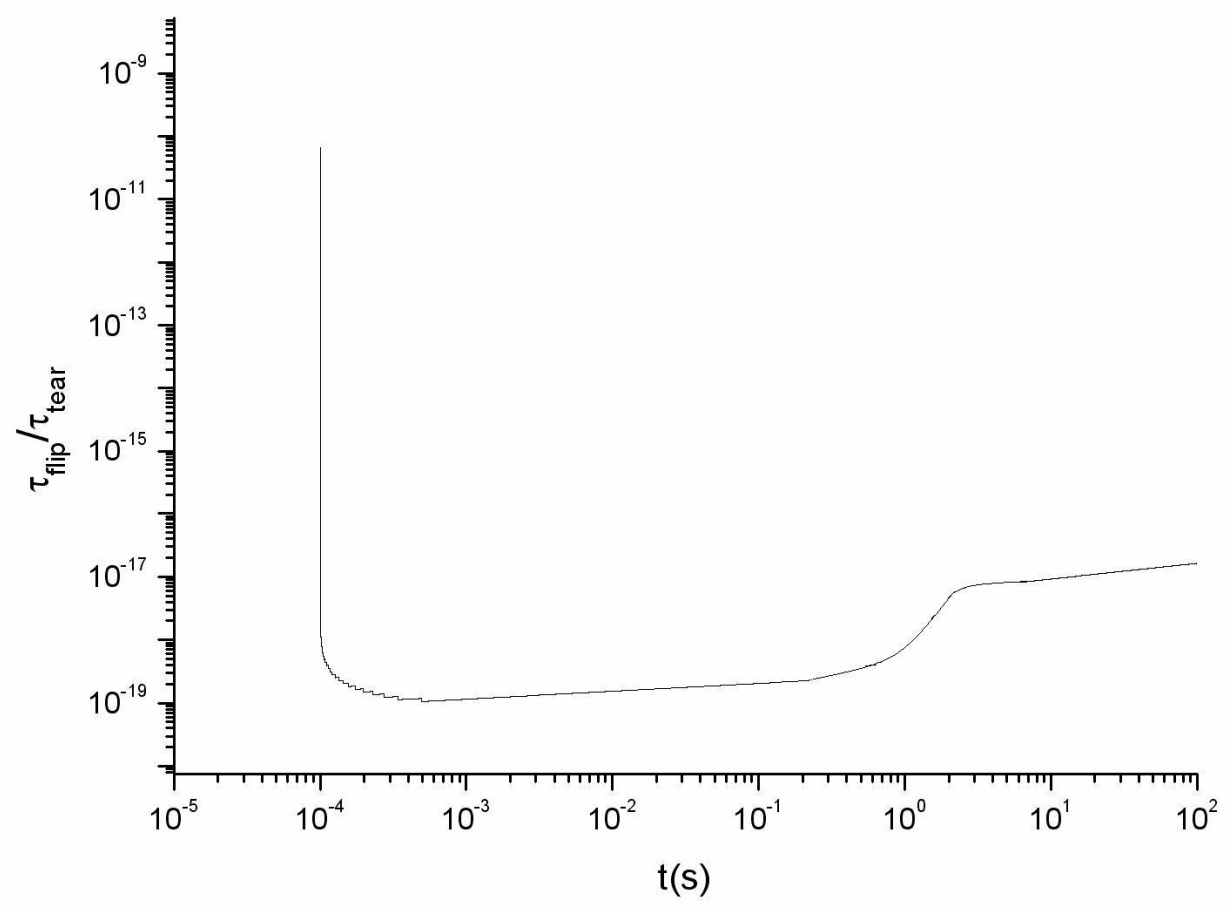

Figura 3.3: Razão entre o tempo de giro $\tau_{\text {flip }}$ e o tempo de reconexão $\tau_{\text {tear }}$ das bolhas magnéticas em função do tempo t(s). 


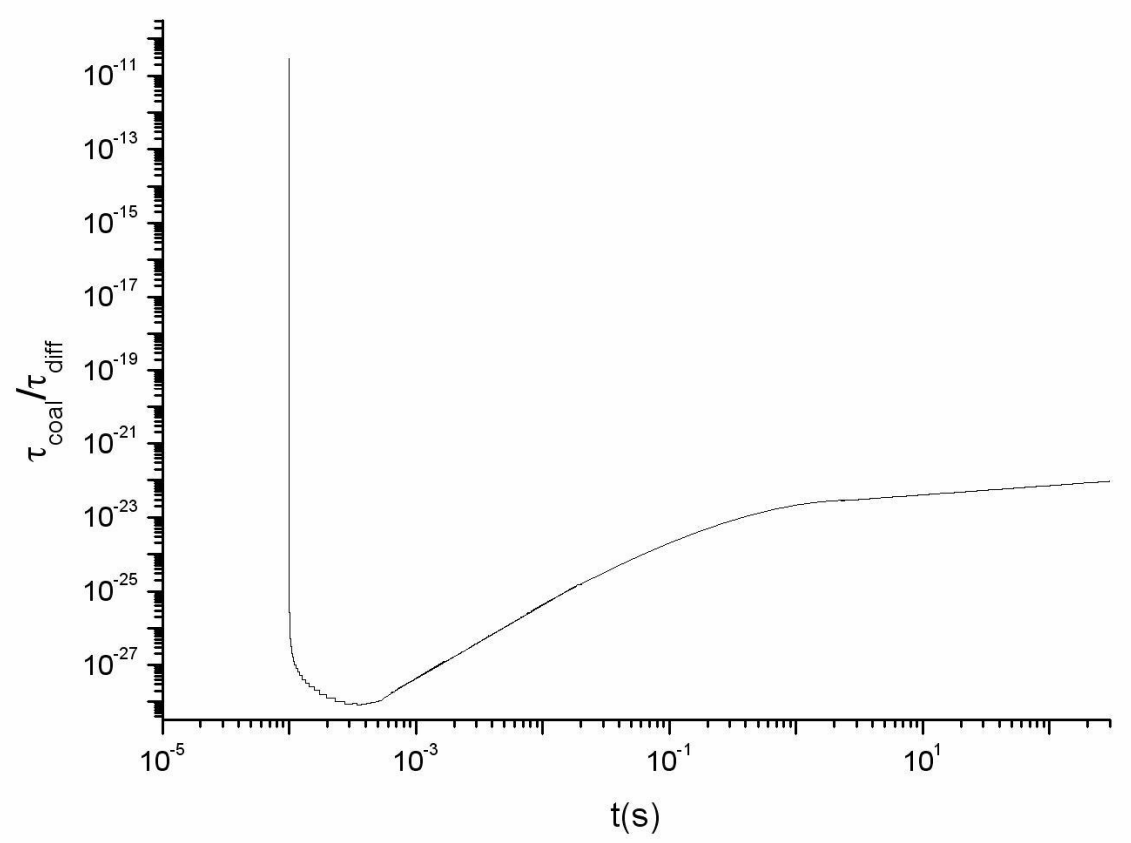

Figura 3.4: Razão entre o tempo de coalescência $\tau_{c o a l}$ e o tempo de difusão das bolhas magnéticas $\tau_{\text {diff }}$. 


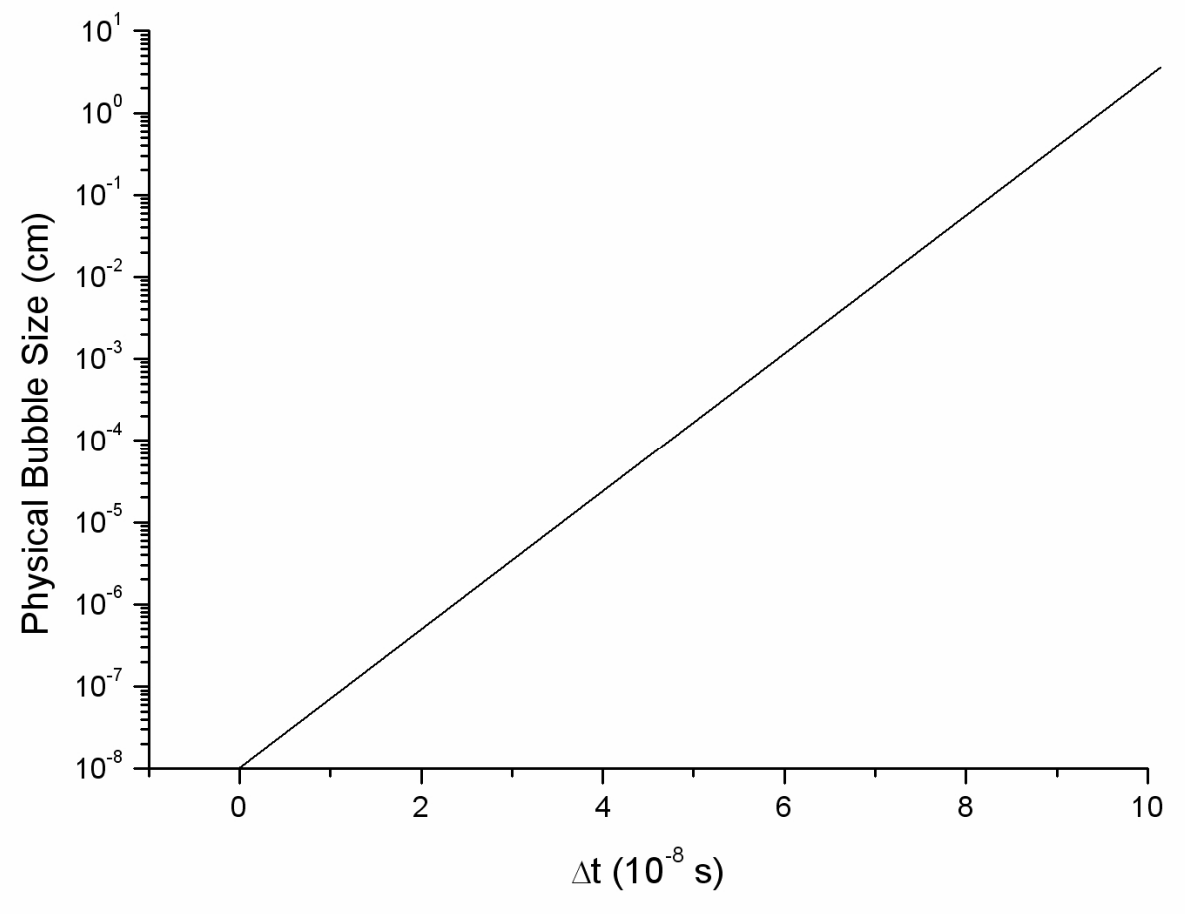

Figura 3.5: Evolução inicial do tamanho físico das bolhas magnéticas, criadas imediatamente após a TFQH, como função do tempo, $t \equiv t_{0}+\Delta t$, para $t_{0}=10^{-4}$ s, e $0<\Delta t\left(10^{-8} s\right) \leq 10$. 


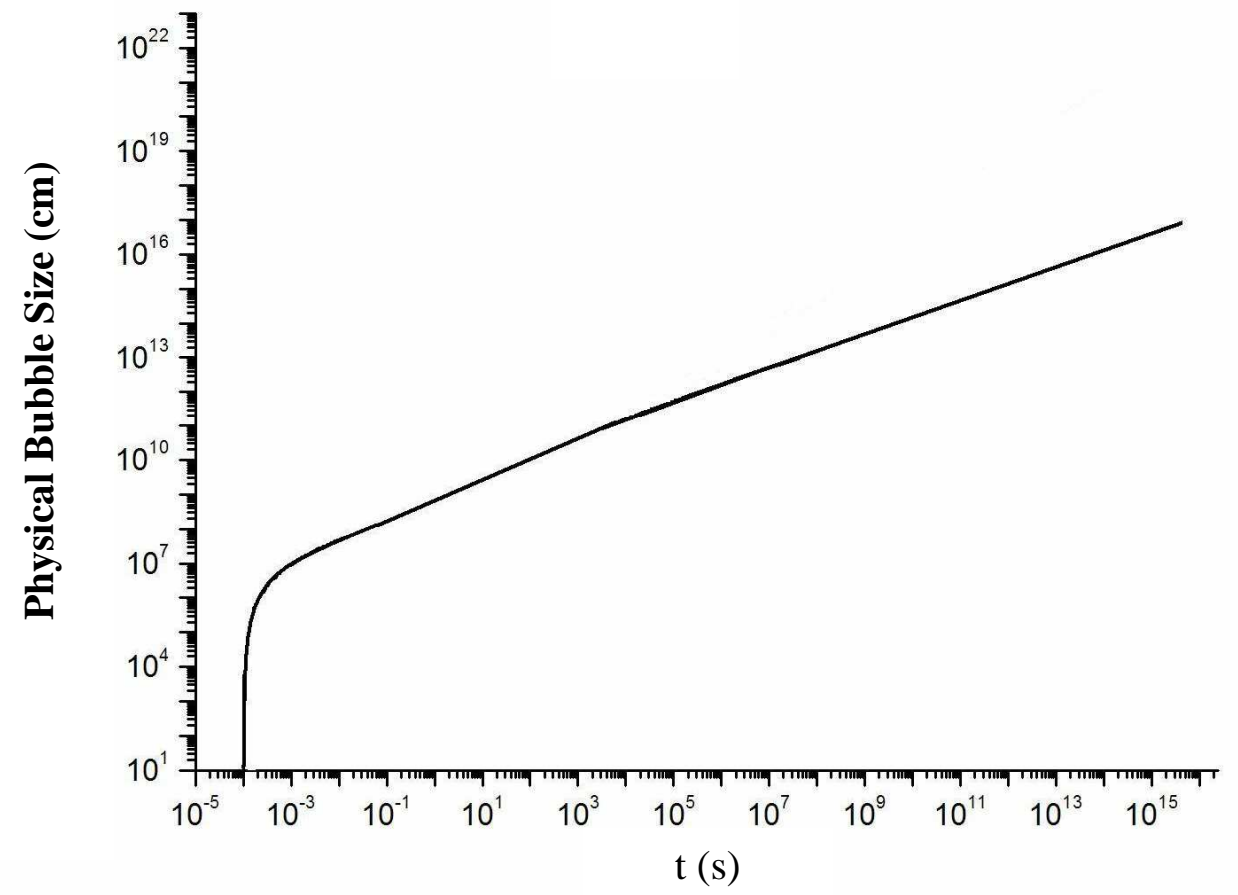

Figura 3.6: Evolução do tamanho físico das bolhas magnéticas em função do tempo $\mathrm{t}$, a partir de $\sim 0.1 s$. 


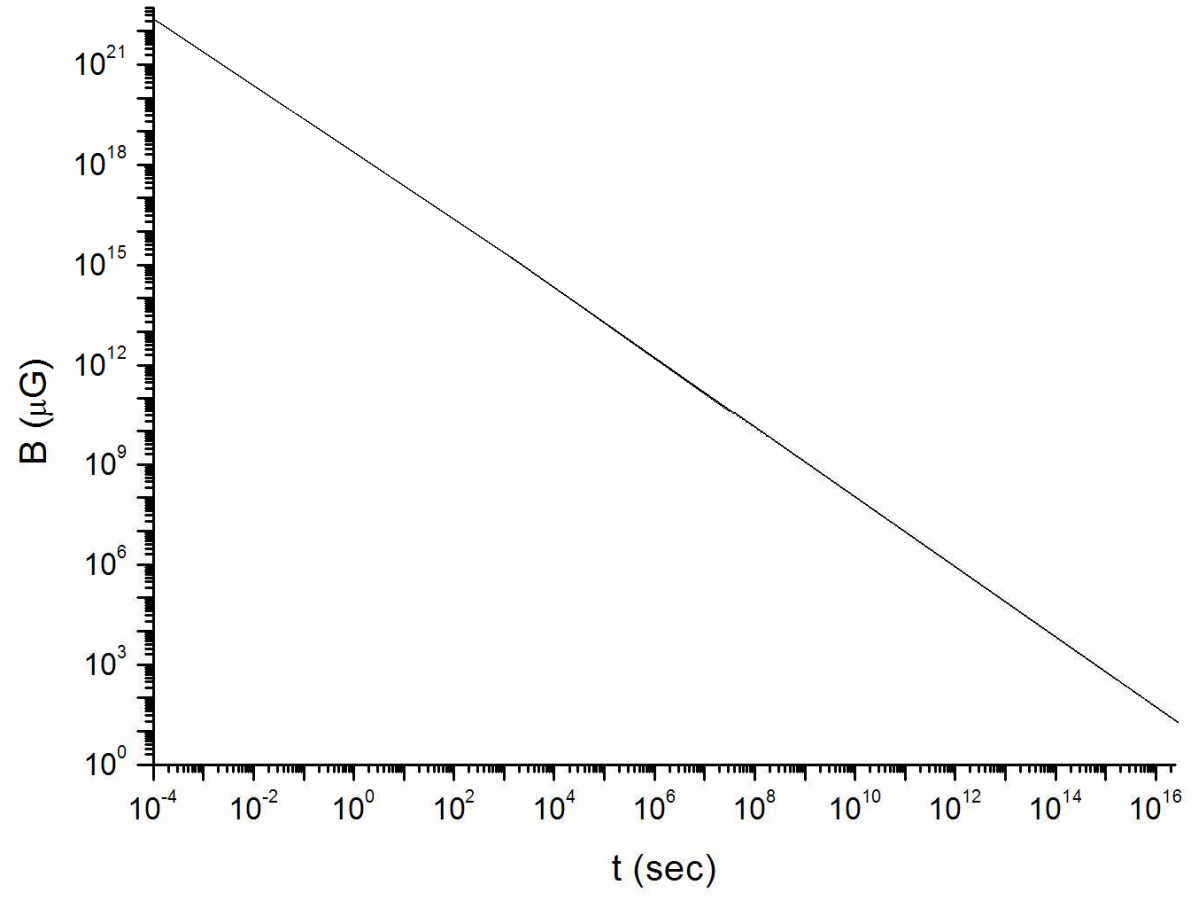

Figura 3.7: Evolução da intensidade do campo magnético $B(\mu \mathrm{G})$ nas bolhas, criado imediatamente após a TFQH, em função do tempo t(s). 


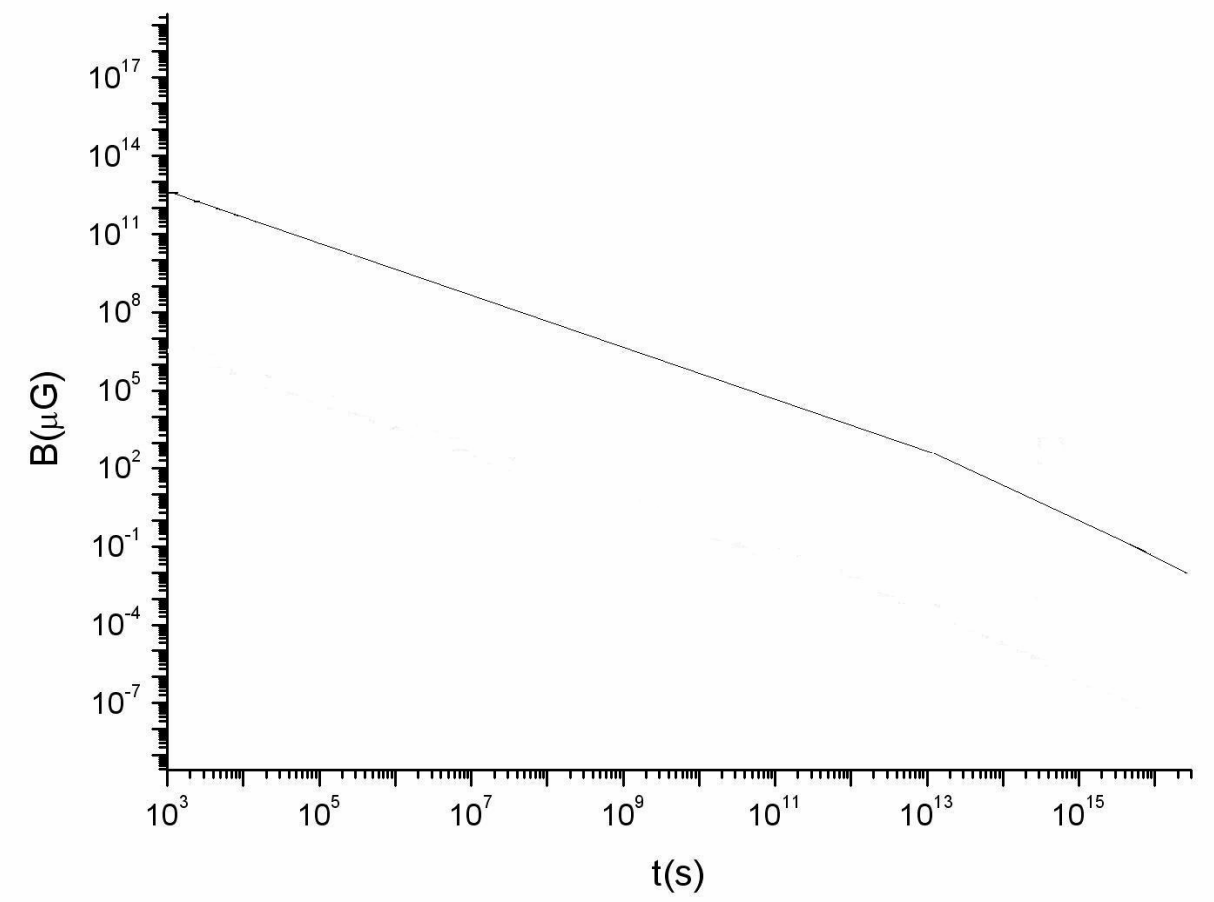

Figura 3.8: Evolução da média do campo magnético $B(\mu G)$ ao longo da linha de visada, sobre uma região comóvel de $\sim 1 \mathrm{Mpc}$ como função do tempo $\mathrm{t}(\mathrm{s})$, desde $t \simeq 3 \times 10^{3} \mathrm{~s}$ até $t \sim 10^{16} \mathrm{~s}(z \sim 10)$, quando surgiram as primeiras galáxias. 
Tabela 3.1: Tamanho e intensidade das bolhas de campo magnético.

\begin{tabular}{|l|c|c|c|c|}
\hline Época & $\mathrm{B}(\mu \mathrm{G})$ & $z$ & $\mathrm{t}(\mathrm{s})$ & $\mathrm{L}(\mathrm{cm})$ \\
\hline Logo após TFQH & $10^{22}$ & $6 \times 10^{11}$ & $10^{-4}$ & $10^{-12}$ \\
\hline Aniquilação $e^{-} e^{+}$ & $10^{18}$ & $10^{10}$ & 1 & $10^{8}$ \\
\hline Nucleossíntese & $10^{15}$ & $10^{8}-10^{9}$ & $1-500$ & $10^{10}$ \\
\hline Equipartição & $2 \times 10^{5}$ & 3600 & $10^{12}$ & $3 \times 10^{14}$ \\
\hline Recombinação & $2 \times 10^{2}$ & 1100 & $8 \times 10^{12}$ & $10^{15}$ \\
\hline Protogaláxias & 9 & $\sim 10$ & $10^{16}$ & $10^{17}$ \\
\hline
\end{tabular}

Tabela 3.2: Média do campo magnético na linha de visada.

\begin{tabular}{|l|c|c|c|c|}
\hline Época & $\mathrm{B}(\mu \mathrm{G})$ & $z$ & $\mathrm{t}(\mathrm{s})$ & $\mathrm{L}(\mathrm{cm})$ \\
\hline Equipartição & $10^{4}$ & 3600 & $10^{12}$ & $10^{18}$ \\
Recombinação & 300 & 1100 & $8 \times 10^{12}$ & $4 \times 10^{22}$ \\
Protogaláxias & $9 \times 10^{-3}$ & $\sim 10$ & $10^{16}$ & $10^{23}$ \\
\hline
\end{tabular}


CAPÍTULO 3. CRIAÇÃO DE CAMPOS MAGNÉTICOS NO UNIVERSO PRIMORDIAL 


\section{Capítulo 4}

\section{Origem dos Campos Magnéticos}

\section{Galácticos}

A existência dos campos magnéticos galácticos foi primeiramente inferida por (Alfvén 1937a,b) e (Fermi 1949), devido às propriedades dos raios cósmicos. Esta inferência foi posteriormente confirmada por dados observacionais de Hiltner (1949) e Hall \& Mikesell (1949). Observações indicam que nossa galáxia possui campos magnéticos com uma componente de grande escala $(\approx \mathrm{kpc})$ com intensidades de vários $\mu \mathrm{G}$. Observamos também campos magnéticos com intensidades e escalas de coerência similares em outras galáxias (Kronberg 1994; Beck 2008). Tradicionalmente, a explicação teórica para os campos magnéticos galácticos foi abordada utilizando-se a teoria de dínamo de campo médio (Parker 1979; Kulsrud \& Zweibel 2008; Steenbeck et al. 1966). A idéia essencial é avaliar a média volumétrica do campo magnético sob duas hipóteses. Primeiro nós assumimos que o campo semente inicial é pequeno, e então durante os estágios iniciais de sua evolução, o campo magnético é fraco e não exerce influência nos movimentos 
hidrodinâmicos. As forças de Lorentz podem então ser negligenciadas e o campo magnético considerado passivo, gerando a chamada aproximação cinemática. Em segundo lugar os campo magnéticos em grandes escalas são considerados muito mais intensos que qualquer flutuação em pequena escala.

Ao longos dos anos, um dos mais populares mecanismo de amplificação de campos magnéticos sementes em discos galácticos é o dínamo $\alpha-\Omega$. Faremos uma breve revisão sobre este mecanismo e algumas críticas feitas a ele. Vamos analisar como dínamos em pequena escala poderiam amplificar de forma mais eficaz nossos campos sementes. Este mecanismo possui uma taxa de amplificação bem maior que o dínamo em larga escala. Utilizamos esta análise, introduzindo como condição inicial, os campos magnéticos previstos por nosso modelo baseado no TFD (de Souza \& Opher 2008). Estudamos como estes campos sementes poderiam explicar os campos magnéticos observados em galáxias.

\subsection{Dínamo $\alpha-\Omega$}

Faremos uma breve revisão sobre a teoria de dínamo de campo médio, introduzida inicialmente por Steenbeck et al. (1966). Consideramos um campo magnético inicial fraco o suficiente, tal que os movimentos no plasma possam ser considerados independentes deste campo magnético (também chamado limite cinemático). Podemos avaliar a evolução do nosso campo magnético através da equação de indução,

$$
\frac{\partial \mathbf{B}}{\partial t}=\nabla \times(\mathbf{v} \times \mathbf{B})+\frac{\eta c^{2}}{4 \pi} \nabla^{2} \mathbf{B} .
$$


Podemos negligenciar o termo de resistividade, como podemos ver com uma simples análise dimensional. Se fizermos a aproximação de $\nabla^{2} \mathbf{B}$ como $\mathbf{B} / L^{2}$ e definirmos o último termo como $\mathbf{B} / t_{d e c}$, podemos escrever o termo de resistividade $\eta c^{2} / 4 \pi \approx$ $10^{7} \mathrm{~cm}^{2} \mathrm{~s}^{-1}$ para um plasma de temperatura $10^{4}$ Kelvin como

$$
t_{d e c} \approx 10^{26} L_{p c}^{2} \text { anos, }
$$

onde $L_{p c}$ é a escala de tamanho em parsecs e $t_{d e c}$ é o tempo de decaimento. Para uma escala $L>10^{12} \mathrm{~cm}$ o tempo de decaimento efetivo é maior que o tempo de Hubble. Logo para análise de teorias sobre origem de campos magnéticos em galáxias, cujas escalas são de vários kpc; o uso da equação

$$
\frac{\partial \mathbf{B}}{\partial t}=\nabla \times(\mathbf{v} \times \mathbf{B})
$$

é perfeitamente plausível.

Podemos escrever o campo de velocidades $\mathbf{v}$ em duas partes: uma associada aos movimentos turbulentos randômicos $\delta \mathbf{v}$, e outra $\mathbf{U}$ que descreve a parte média e coerente. Para o caso galáctico $\mathbf{U}$ é a rotação diferencial da galáxia.

$$
\mathbf{v}=\mathbf{U}+\delta \mathbf{v}
$$

Podemos escrever o campo magnético como uma combinação de um termo médio $\overline{\mathbf{B}}$ e uma parte randômica $\delta \mathbf{B}$, então

$$
\mathbf{B}=\overline{\mathbf{B}}+\delta \mathbf{B} .
$$


Substituindo na equação (4.3) e realizando uma média sobre os termos turbulentos, temos

$$
\frac{\partial \overline{\mathbf{B}}}{\partial t}=\nabla \times(\mathbf{U} \times \overline{\mathbf{B}})+\nabla \times(\langle\delta \mathbf{v} \times \delta \mathbf{B}\rangle)
$$

Podemos ver o surgimento de um termo extra na equação anterior associado com os movimentos randômicos. O termo devido aos campos randômicos pode ser escrito como

$$
\langle\delta \mathbf{v} \times \delta \mathbf{B}\rangle=\alpha \overline{\mathbf{B}}-\beta \nabla \times \overline{\mathbf{B}},
$$

onde os termos $\alpha$ e $\beta$ podem ser definidos da forma

$$
\begin{aligned}
& \alpha=-\frac{\tau}{3}\langle\mathbf{v} \cdot \nabla \times \mathbf{v}\rangle, \\
& \beta=\frac{\tau}{2}\left\langle\mathbf{v}^{2}\right\rangle
\end{aligned}
$$

sendo $\tau$ é o tempo de correlação dos movimentos turbulentos (Kulsrud 2005). Podemos substituir estes termos na (4.7), chegando na famosa equação para o dínamo $\alpha-\Omega$

$$
\frac{\partial \overline{\mathbf{B}}}{\partial t}=\nabla \times(\mathbf{U} \times \overline{\mathbf{B}})+\nabla \times(\alpha \overline{\mathbf{B}})+\beta \nabla^{2} \overline{\mathbf{B}} .
$$

Podemos aplicar esta equação ao disco galáctico, onde podemos usar coordenadas cilíndricas $r, \theta, z$, e mantendo derivadas apenas da direção fina do disco, $z$, teremos

$$
\begin{aligned}
\frac{\partial B_{r}}{\partial t} & =-\frac{\partial}{\partial z}\left(\alpha B_{\theta}\right)+\beta \frac{\partial^{2} B_{r}}{\partial z^{2}} \\
\frac{\partial B_{\theta}}{\partial t} & =-\Omega B_{r}+\beta \frac{\partial^{2} B_{\theta}}{\partial z^{2}} .
\end{aligned}
$$


Nós substituímos a expressão para rotação galáctica $r \Omega \hat{\theta}$ no lugar de $\mathbf{U}$, onde $\Omega$ é a velocidade angular em um raio $r$, usamos o fato de que $r \Omega$ é uma constante e achamos assim a expressão $d \Omega / d r=-\Omega / r$. Podemos descartar o termo $\partial_{z}\left(\alpha B_{r}\right)$ na equação para $B_{\theta}$, pois este é negligenciável em relação a rotação diferencial.

O procedimento usual para resolver a equação (4.11) é olhar para os modos de crescimento, proporcionais a $e^{\gamma t}$ e resolver um problema de autovalores. As condições iniciais que normalmente são utilizadas, assumem que o disco está confinado a uma região $-h<z<h$. Outra hipótese é que o coeficiente $\beta$ é muito grande fora do disco, e o campo magnético é tomado como zero para $|z|>h$. Como conseqüência, $B_{r}$ e $B_{\theta}$ se anulam para $|z|=h$. Estas hipóteses são chamadas de condições de contorno de vácuo. Estes pressupostos são de extrema importância para a validade desta teoria.

Podemos ver que estas condições estão diretamente relacionadas à mudança de fluxo no disco, integrando a equação (4.11)

$$
\frac{d}{d t} \int_{-h}^{+h} B_{r}=\left.\beta \frac{\partial B_{r}}{\partial z}\right|_{-h} ^{+h}-\left.\alpha B_{\theta}\right|_{-h} ^{+h},
$$

onde $B_{r}, B_{\theta}$ são considerados simétricos. Podemos ver que o primeiro termo $-\beta \partial B_{r} / \partial z$ é a taxa de escape do fluxo de $B_{r}$ através da região de contorno. Devido as condições de contorno para $B_{r}$ e $B_{\theta}$, o segundo termo é nulo. Embora neste caso as linhas de fluxo do campo magnético não estejam congeladas no plasma, o fluxo total dentro do disco continua satisfazendo a relação de conservação (4.12). O fluxo de $B_{\theta}$ não é conservado per se, uma vez que ele cresce conforme o fluxo de $B_{r}$ se esvai, contudo isto não muda o número total de linhas de força. Estas condições de contorno são necessárias se imaginamos que o fluxo que alcança as bordas 
do disco escapa instantaneamente. Contudo, é difícil imaginar que esta situação seja fisicamente realista. O congelamento do fluxo implica que qualquer fluxo que escape do disco, estará imerso no meio interestelar. Logo, para o dínamo trabalhar, uma grande porção do meio interestelar precisa ser removida a cada efolding do campo magnético. Considere que uma fração $f$ do meio interestelar seja removida a cada aumento exponencial do campo magnético. Então, se a intensidade do campo aumenta de $B_{0}$ até $B_{1}$, a massa do meio interestelar precisa diminuir de $M_{0}$ até $M_{1}=M_{0}\left(B_{1} / B_{0}\right)^{-f}$. Para amplificarmos um campo de $B_{0}=10^{-16} \mathrm{G}$ para $B_{1}=10^{-6} \mathrm{G}$ com $f=1 / 3$ deveríamos precisar de $M_{0} \sim 2000 M_{1}$, sendo um processo pouco provável de ocorrer (Kulsrud \& Zweibel 2008).

Podemos obter uma idéia aproximada do tamanho de $\beta$ no meio interestelar, levando em conta a velocidade turbulenta randômica $\delta v \approx 10 \mathrm{~km} / \mathrm{s}$, e o comprimento de correlação $\delta v \tau \approx 100$ pc, (Parker 1979; Ruzmaikin et al. 1988). Que nos dá $\beta \approx 1.5 \times 10^{26} \mathrm{~cm}^{2} / \mathrm{s}$. Um possível valor para altura do disco galáctico é $h \sim 300$ pc, resultando em uma taxa de crescimento do dínamo da ordem de $1.5 \times 10^{16} \mathrm{~s}=5 \times 10^{8}$ anos.

Para obter um campo magnético da ordem de $\sim \mu G$ em $\sim 10$ bilhões de anos, precisamos iniciar com um campo semente de $\sim 10^{-13} \mathrm{G}$. Observações indicam a presença de campos magnéticos de $\sim \mu \mathrm{G}$ em aglomerados de galáxias e galáxias em alto desvio para o vermelho $z$ (Perry 1994; Kronberg 1994). Isto é difícil de explicar com a teoria de dínamo galáctica $\alpha-\Omega$ (Zweibel \& Heiles 1997).

Como podemos ver, existem alguns problemas cruciais com a teoria clássica $\alpha-$ $\Omega$, sendo necessário uma investigação mais profunda de como um campo magnético semente pode evoluir ao longo da formação dos discos galácticos. 


\subsection{Turbulência em Pequena Escala}

Podemos pensar em duas escalas de atuação dos dínamos. Podemos dividi-los em dínamos de pequena e grande escala. Os de grande escala mostram grande escala espacial de coerência. Eles também mostram um termo de alta ordem temporal, onde seu ciclo em geral é muito maior que a escala de tempo dos movimentos turbulentos. Dínamos em pequena escala produzem campos magnéticos correlacionados em escalas da ordem ou menores que a escala de energia transportada pelo campo de velocidade. Estes dínamos em pequena escala são importantes por várias razões. Eles têm uma taxa de crescimento muito maior que os dínamos de grande escala. Eles podem ser muito importantes em regiões onde o dínamo de grande escala não atua, tais como aglomerados de galáxias e galáxias elípticas, onde os efeitos de rotação são desprezíveis.

A dinâmica das flutuações magnéticas pode ser melhor estudada em termos da dinâmica de suas funções de correlação. Kazantsev (1968) derivou as equações para as correlações longitudinais em um plasma isotrópico, homogêneo, assumindo uma turbulência Markoviana e sem helicidade média. Vainshtein \& Kichatinov (1986) incorporou os efeitos de helicidade e derivou as equações para correlações tanto longitudinais quando helicoidais.

Subramanian (1999) e Brandenburg \& Subramanian (2000) derivaram a evolução das equações para as correlações magnéticas, levando em conta os efeitos de back reaction devido às forças de Lorentz, causada pela difusão ambipolar. Utilizamos em nosso trabalho a abordagem discutida acima, incluindo como condição inicial, o campo magnético semente previsto pelo TFD. Analisamos como estes campos podem ser amplificados pela turbulência gerada na região protogaláctica. 
A equação para evolução do campo magnético em um gás parcialmente ionizado é governado pela equação de indução,

$$
(\partial \mathbf{B} / \partial t)=\nabla \times(\mathbf{v} \times \mathbf{B}-\eta \nabla \times \mathbf{B}),
$$

onde $\mathbf{B}$ é o campo magnético, $\mathbf{v}$ é a velocidade da componente iônica do fluido e $\eta$ é a resistividade ohmica. Os íons experimentam uma força de Lorentz devido ao campo magnético. Isto vai causar uma difusão em relação aos componentes neutros do fluido. Se as colisões de íons-neutros são rápidas o suficiente, podemos assumir que a força de Lorentz nos íons é balanceada pela fricção com os neutros. Sobre esta aproximação, a equação de Euler para os íons torna-se

$$
\rho_{i} \nu_{i n}\left(\mathbf{v}_{i}-\mathbf{v}_{n}\right)=[(\nabla \times \mathbf{B}) \times \mathbf{B}] /(4 \pi),
$$

onde $\rho_{i}$ é a densidade de massa dos íons, $\nu_{i n}$ a freqüência de colisão íons-neutros e $\mathbf{v}_{n}$ é a velocidade de partículas neutras. Definimos a velocidade de difusão ambipolar $\mathbf{v}_{D}$, como $\left(\mathbf{v}_{i}-\mathbf{v}_{n}\right)$.

Usando a equação de Euler para os íons e substituindo por $\mathbf{v}_{i}$, a equação de indução torna-se uma equação não linear

$$
\frac{\partial \mathbf{B}}{\partial t}=\nabla \times\left[\mathbf{v}_{n} \times \mathbf{B}+a[((\nabla \times \mathbf{B}) \times \mathbf{B}) \times \mathbf{B}]-\eta \nabla \times \mathbf{B}\right],
$$

onde definimos

$$
a=\frac{1}{4 \pi \rho_{i} \nu_{i n}} .
$$

O campo de velocidade é assumido ser independente do campo magnético. Vamos considerar que $\mathbf{v}_{n}$ tem uma componente estocástica $\mathbf{v}_{T}$, além da componente 
média $\mathbf{v}_{0}$, isto é $\mathbf{v}_{n}=\mathbf{v}_{0}+\mathbf{v}_{T}$. Uma vez que $\mathbf{v}_{T}$ é estocástica a equação (4.15) torna-se uma equação diferencial a derivadas parciais estocástica. Sua solução depende de propriedades estatísticas do campo de velocidades $\mathbf{v}_{T}$.

No caso em que a velocidade turbulenta $\mathbf{v}_{T}$ é isotrópica, homogênea, com um campo randômico gaussiano de média nula, podemos definir a função de correlação de dois pontos do campo de velocidades da forma $\left\langle v_{T}^{i}(x, t) v_{T}^{j}(y, s)\right\rangle=T^{i j}(r) \delta(t-s)$, com

$$
T^{i j}(r)=T_{N N}\left[\delta^{i j}-\frac{r^{i} r^{j}}{r^{2}}\right]+T_{L L} \frac{r^{i} r^{j}}{r^{2}}+C \epsilon_{i j f} r^{f} .
$$

O símbolo \langle\rangle representa a média sobre as velocidades estocásticas, $r=|x-y|$, $r^{i}=x^{i}-y^{i}$, onde $T_{L L}(r)$ e $T_{N N}(r)$ são as correlações longitudinais e transversas do campo de velocidades e C(r) representa a parte helicoidal das correlações de velocidades. Se $\mathbf{v}_{T}$ é assumido ter divergência nula, então

$$
T_{N N}(r)=\frac{1}{2 r} \frac{\partial}{\partial r}\left(r^{2} T_{L L}(r)\right)
$$

com

$$
T_{L L}(0)=\frac{1}{3} \int_{0}^{t}\left\langle v(t) \cdot v\left(t^{\prime}\right)\right\rangle d t^{\prime}
$$

onde $T_{L}(0)$ é o coeficiente de difusão turbulenta para o campo médio.

Considerando as flutuações isotrópicas e homogêneas, podemos escrever a correlação de dois pontos para o campo magnético como,

$$
\left\langle B^{i}(\mathbf{x}, t) B^{j}(\mathbf{y}, t)\right\rangle=M^{i j}(r, t),
$$

onde 


$$
M^{i j}=M_{N}\left[\delta^{i j}-\left(\frac{r^{i} r^{j}}{r^{2}}\right)\right]+M_{L}\left(\frac{r^{i} r^{j}}{r^{2}}\right)+H \epsilon_{i j k} r^{k},
$$

(Subramanian 1999). $M_{L}(r, t)$ e $M_{N}(r, t)$ são as correlações longitudinais e transversais do campo magnético e $H(r, t)$ é o termo de correlação helicoidal.

A equação da indução magnética pode ser escrita como uma equação pra evolução de $M_{L}$ e $H$ (mais detalhes sobre a dedução no apêndice B)

$$
\begin{aligned}
\frac{\partial M_{L}}{\partial t}(r, t) & =\frac{2}{r^{4}} \frac{\partial}{\partial r}\left(r^{4} \kappa_{N}(r, t) \frac{\partial M_{L}(r, t)}{\partial r}\right) \\
& +G(r) M_{L}(r, t)+4 \alpha_{N} H(r, t), \\
\frac{\partial H}{\partial t}(r, t) & =\frac{1}{r^{4}} \frac{\partial}{\partial r}\left[r ^ { 4 } \frac { \partial } { \partial r } \left[2 \kappa_{N}(r, t) H(r, t)\right.\right. \\
& \left.\left.-\alpha_{N}(r, t) M_{L}(r, t)\right]\right],
\end{aligned}
$$

onde

$$
\begin{gathered}
\kappa_{N}(r, t)=\eta+T_{L L}(0)-T_{L L}(r)+2 a M_{L}(0, t), \\
\alpha_{N}(r, t)=2 C(0)-2 C(r)-4 a H(0, t),
\end{gathered}
$$

e

$$
G(r)=-4\left\{\frac{d}{d r}\left[\frac{T_{N N}(r)}{r}\right]+\frac{1}{r^{2}} \frac{d}{d r}\left[r T_{L L}(r)\right]\right\},
$$

(Subramanian 1999). Estas equações formam um conjunto fechado de equações diferenciais para evolução de $M_{L}$ e $H$, descrevendo a evolução das correlações magnéticas em pequena e grande escala.

Os temos envolvendo $\kappa_{N}$ nas equações (4.22) e (4.23), representam os efeitos 
da difusão nas correlações magnéticas. O coeficiente de difusão inclui os efeitos da difusão microscópica $\eta$ e a difusão turbulenta dependente da escala $T_{L L}(0)-T_{L L}(r)$. O efeito da difusão ambipolar, sob esta aproximação gaussiana, adiciona uma quantidade $2 a M_{L}(0, t)$ ao coeficiente de difusão; o termo é proporcional à densidade de energia das flutuações do campo. Analogamente $\alpha_{N}$ representa a dependência da escala do efeito $\alpha,(2 C(0)-2 C(r))$ e o efeito da difusão ambipolar é diminuir por um fator $4 a H(0, t)$ a quantidade proporcional à helicidade de corrente média das flutuações do campo.

\subsection{Turbulência Protogaláctica}

Devido às inúmeras dificuldades com o modelo $\alpha-\Omega$, para explicar a origem dos campos magnéticos galácticos, é interessante considerar a possibilidade destes campos serem gerados durante a época na qual a própria galáxia se formou. Esta teoria foi inicialmente proposta por Pudritz \& Silk (1989). A idéia principal é que devemos ter uma turbulência considerável gerada durante o colapso do plasma cósmico que gera a protogaláxia. Ela ocorre devido às ondas de choque originadas pelas instabilidades geradas pelo colapso gravitacional. Este processo cria uma turbulência do tipo Kolmogorov (Kulsrud \& Zweibel 2008).

A teoria de Kolmogorov é muito útil por ser de fácil aplicação e muito bem suportada tanto observacionalmente quanto em simulações numéricas. Podemos imaginar uma injeção de energia em um fluido, como por exemplo o meio interestelar, através de estrelas ou supernovas, em grandes escalas. Esta energia em geral é inserida através de grandes vórtices. Estes vórtices se quebram em vórtices com metade do tamanho original até uma escala onde os efeitos de viscosidade 
destroem os vórtices mais rápido do que eles são capazes de transferir energia para vórtices ainda menores, denominada escala de corte da turbulência. A razão entre a escala dos maiores vórtices e a escala de corte devido a viscosidade em geral é muito grande, e a faixa entre estas duas escalas é denominada faixa inercial. Nesta faixa esperamos que a turbulência seja igual em todas as escalas. Isto têm sido estabelecido por várias simulações numéricas de formação de estruturas (Ryu et al. 1993; Kang et al. 1994). Uma das características da turbulência de Kolmogorov é a existência de um grande número de vórtices, em várias escalas. Grandes vórtices dão surgimento a vórtices cada vez menores, até a escala de corte dada pela viscosidade do meio $L_{v i s c}$. A idéia principal é que uma dada fração de energia $E$, seja transferida de grandes para pequenos vórtices. O regime onde ocorre a turbulência pode ser estabelecido entre a região do maior vórtice $L$, e a escala de corte $L_{v i s c}$. O maior vórtice em nossa análise é comparável ao tamanho da protogaláxia (Schekochihin et al. 2002) .

Podemos desprezar o termo de acoplamento $\alpha_{N} H$ com excelente acurácia, em nossa análise da evolução de $M_{L}$ (Brandenburg \& Subramanian 2005). Para o dínamo em pequena escala, considerando uma turbulência do tipo Kolmogorov, podemos modelar $T_{L L}(r)$ da forma (Brandenburg \& Subramanian 2005):

$$
\begin{array}{rl}
T_{L L}(r)=\frac{V_{c} L_{c}}{3}\left[1-R_{e}^{1 / 2}\left(\frac{r}{L}\right)^{2}\right] & 0<r<l_{c} \\
T_{L L}(r)=\frac{V_{c} L_{c}}{3}\left[1-\left(\frac{r}{L}\right)^{4 / 3}\right] & l_{c}<r<L, \\
T_{L L}(r)=0 & r>L,
\end{array}
$$

onde $R_{e}=V L / \nu$ é o número Reynolds hidrodinâmico, e $\nu$ é a viscosidade cinemá- 
tica, $l_{c} \sim L R_{e}^{-3 / 4}$ é a escala de corte devido a viscosidade, L e V são o tamanho e velocidades do maior vórtice turbulento respectivamente.

Supondo que os movimentos turbulentos ocorram em uma dada escala $L$, com uma velocidade $V$, podemos definir o número de Reynolds magnético $R_{m}=V L / \eta$, onde $\eta$ é a resistividade do meio. Para turbulência Kolmogorov, a velocidade dos vórtices turbulentos em uma dada escala $l$, é $v_{l} \propto l^{1 / 3}$, dentro da faixa inercial. Logo a dependência com a escala do coeficiente de difusão é $v_{l} l \propto l^{4 / 3}$. Esta forma de lei de potência característica do espectro de Kolmogorov serviu de motivação para a modelagem de $T_{L L}$. Usando esta relação podemos definir o número de Reynolds magnético associado com uma dada escala $l$, como $R_{m}(l)=v_{l} l / \eta=R_{m}\left(l / L_{c}\right)^{4 / 3}$. Esperamos que a taxa de crescimento do campo magnético $\Gamma$, referente a uma dada escala $l$ seja $\Gamma_{l} \sim v_{l} / l \propto l^{-2 / 3}$.

Como estamos interessados em avaliar a amplificação do nosso campo semente na era de formação das galáxias, precisamos dos parâmetros associados ao plasma nesta época. O gás é muito quente em protogaláxias, podendo ser considerado totalmente ionizado (Schekochihin et al. 2002). O número de Reynolds é muito grande, logo a turbulência se desenvolve em uma grande faixa de escalas, desde a maior escala da ordem do tamanho da protogaláxia, terminando na escala de corte devido a viscosidade.

Consideramos valores da literatura como modelo fiducial (Malyshkin \& Kulsrud 2002; Schekochihin et al. 2002). Variamos estes parâmetros de forma a analisar a sensibilidade do nosso resultado em relação às condições iniciais. Valores típicos da massa total $M$ da protogaláxia são de $\sim 10^{12} M_{\odot}$, sua temperatura é da ordem de $\mathrm{T} \sim 10^{6} \mathrm{~K}$, o tamanho típico é $L_{P G} \sim 200 \mathrm{kpc}$. A viscosidade cinemática é $\sim 10^{7} \mathrm{~cm} / \mathrm{s}$, a resistividade de Spitzer $\eta_{s}=6.53 \times 10^{12} T^{-3 / 2} \ln \Lambda \mathrm{cm}^{2} \mathrm{~s}^{-1} \sim 8 \times$ 
$10^{4} \mathrm{~cm}^{2} \mathrm{~s}^{-1}$. As velocidades típicas dos maiores vórtices são da ordem de $V_{P G} \sim 10^{7}$.

Nós resolvemos numericamente a equação (4.22) utilizando como condição inicial nosso espectro previsto para um campo magnético sobre uma determinada escala $r$, (de Souza \& Opher 2008). Nas figuras 4.1, 4.2, 4.3 e 4.4 avaliamos $M_{L}$ para os parâmetros característicos das protogaláxias.

Podemos fazer uma conta simples para entender o papel da turbulência protogaláctica na amplificação dos nossos campos sementes no espaço de número de onda $k$. Podemos escrever o espectro de vorticidade da forma

$$
\omega^{2}=\int J(k) d k .
$$

O espectro de vorticidade terá uma dependência com o número de onda $k, \omega(k)$. O espectro será dado por $k$ vezes a velocidade prevista pelo espectro de Kolmogorov,

$$
\omega^{2}(k)=k J(k)=k_{0}^{2 / 3} k^{4 / 3} v_{0}^{2},
$$

$\log 0$

$$
\omega=\omega_{0}\left(\frac{k}{k_{0}}\right)^{2 / 3},
$$

onde $\omega_{0}=k_{0} v_{0}$ é a vorticidade na maior escala $k_{0}$.

Assumindo que não existe acoplamento entre as diversas escalas, considerando o dínamo gerado pela vorticidade e o termo de difusão causado pelo relaxamento da tensão das linhas de campo magnéticas, que se dá a uma taxa proporcional à velocidade Alfvén, podemos escrever a taxa de variação do campo magnético para 
um dado número de onda $k$ da forma,

$$
\frac{d B(k)}{d t}=\omega(k) B(k)-\frac{k B^{2}(k)}{\sqrt{4 \pi \rho}} .
$$

Para resolver esta equação podemos usar como condição inicial nosso campo semente calculado no capítulo anterior, $B(L)=10^{-5}(0.1 p c / r)^{3 / 2} G$. A solução da equação (4.33) para um dado valor de campo magnético inicial $B_{0}$ será

$$
B(k, t)=\frac{B_{0} e^{\omega(k) t} \sqrt{4 \pi \rho}}{B_{0} k\left(e^{\omega(k) t}-1\right)+\omega(k) \sqrt{4 \pi \rho}} .
$$




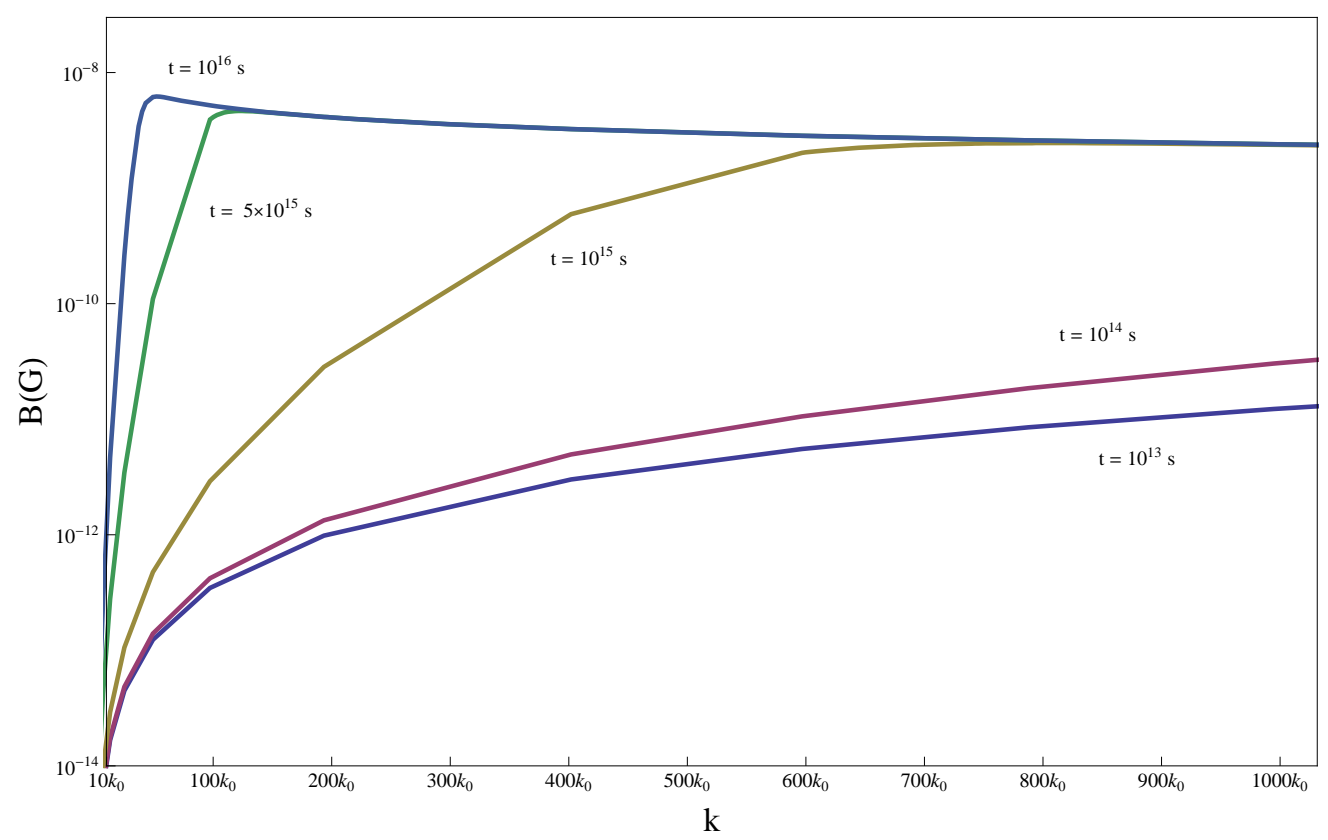

Figura 4.1: Valores de $B(k)$ como função do número de onda $k_{0}\left(k_{0}=2 \pi / L_{P G}\right)$ em diversos instantes de tempo. 


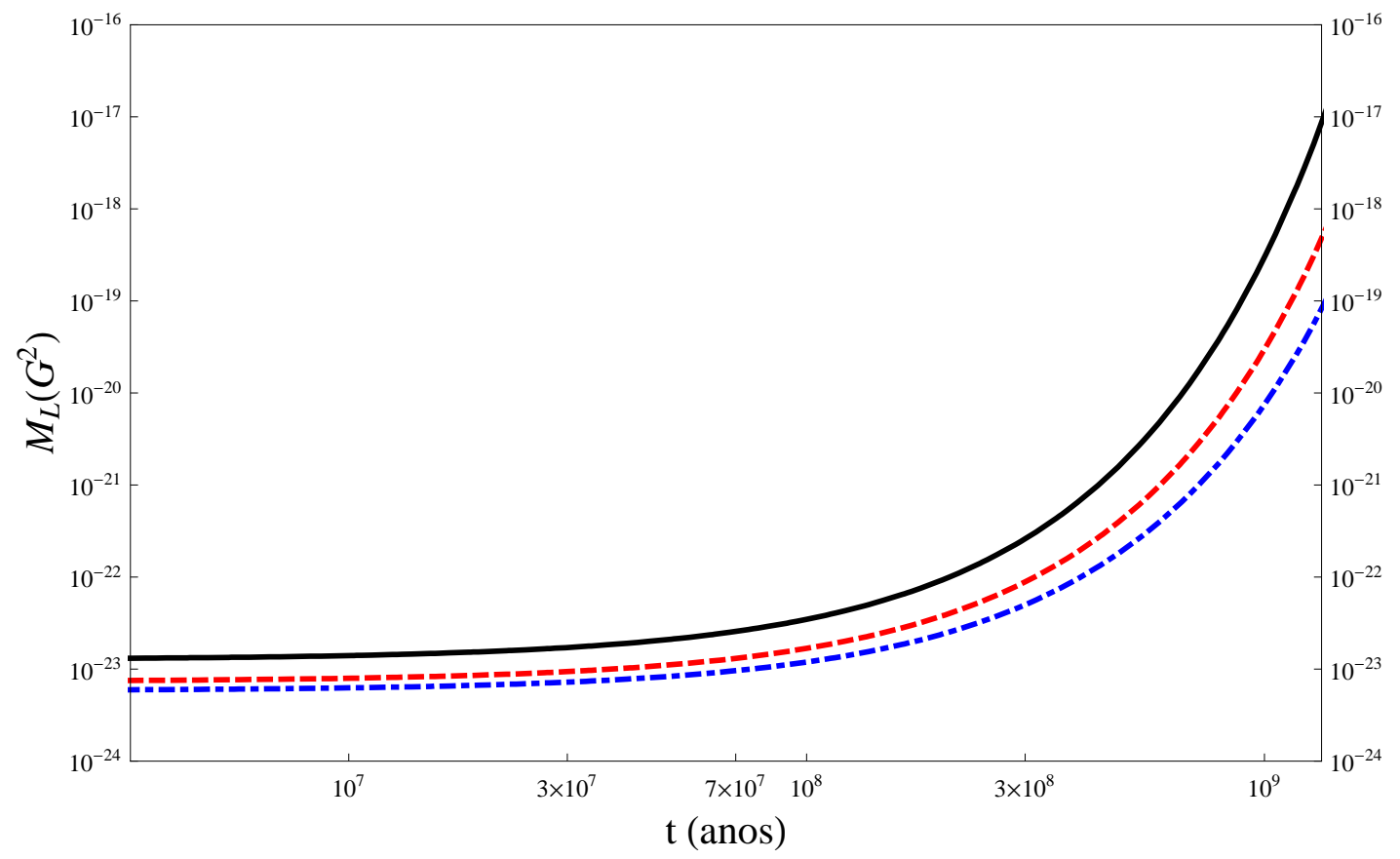

Figura 4.2: Valores de $M_{L}\left(G^{2}\right)$ como função do tempo t (anos). A curva preta contínua representa os valores de referência: $L_{c}=200 \mathrm{kpc}, \mathrm{r}=3 \mathrm{kpc}, V_{c}=10^{7} \mathrm{cms}^{-1}$ e $M_{L}(r, 0)=10^{-11}(0.1 p c / r)^{3} G^{2}$. Nós variamos $\mathrm{r}$ : curva vermelha tracejada $r=4 k p c$, curva azul ponto-traço-ponto $r=5 k p c$. 


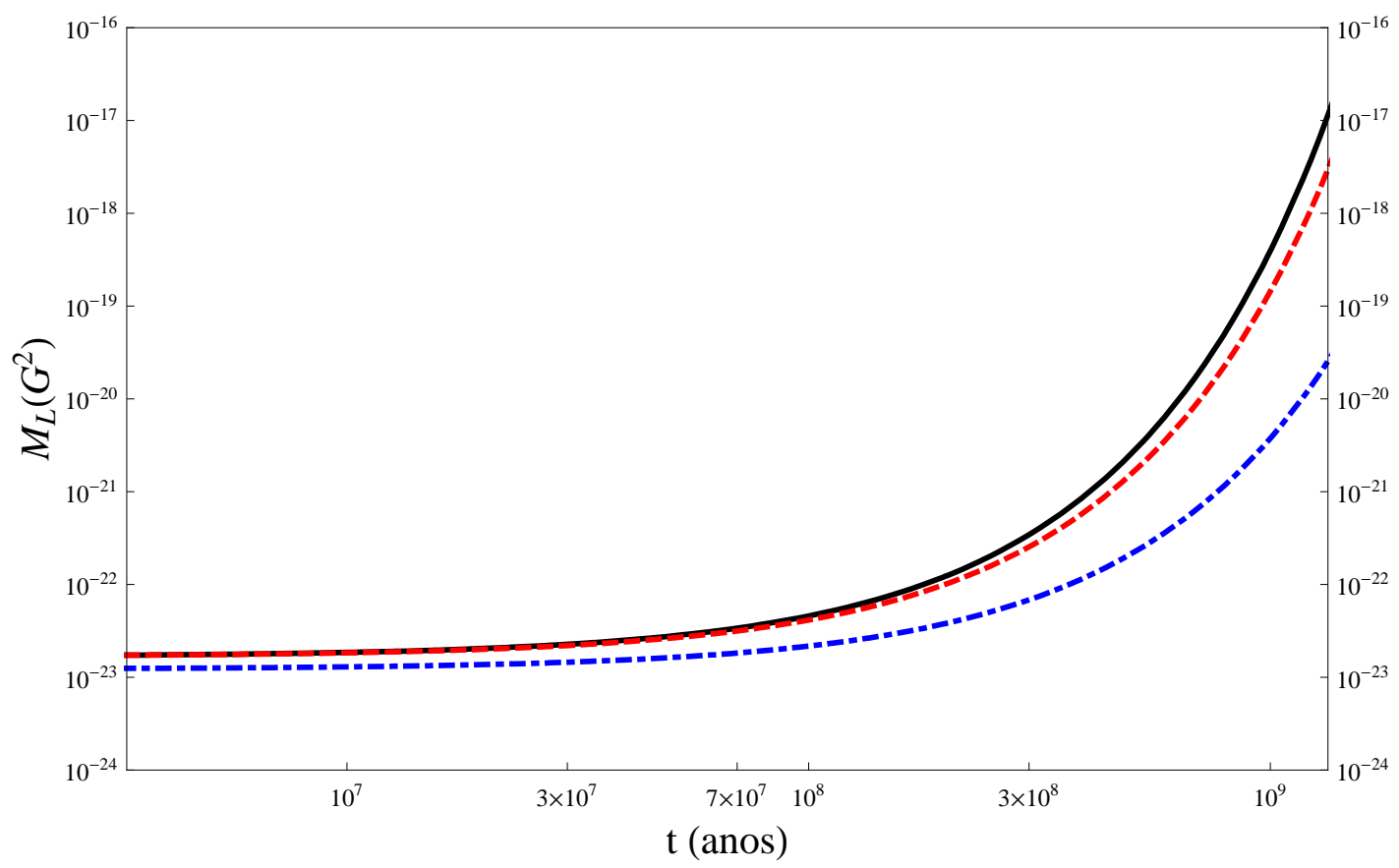

Figura 4.3: Valores de $M_{L}\left(G^{2}\right)$ como função do tempo t(anos). A curva preta contínua representa os valores de referência (veja fig. 4.2). Nós variamos $V_{c}$ : curva vermelha tracejada $V_{c}=8 \times 10^{6} \mathrm{~cm} / \mathrm{s}$, curva azul ponto-traço-ponto $V_{c}=$ $6 \times 10^{6} \mathrm{~cm} / \mathrm{s}$. 


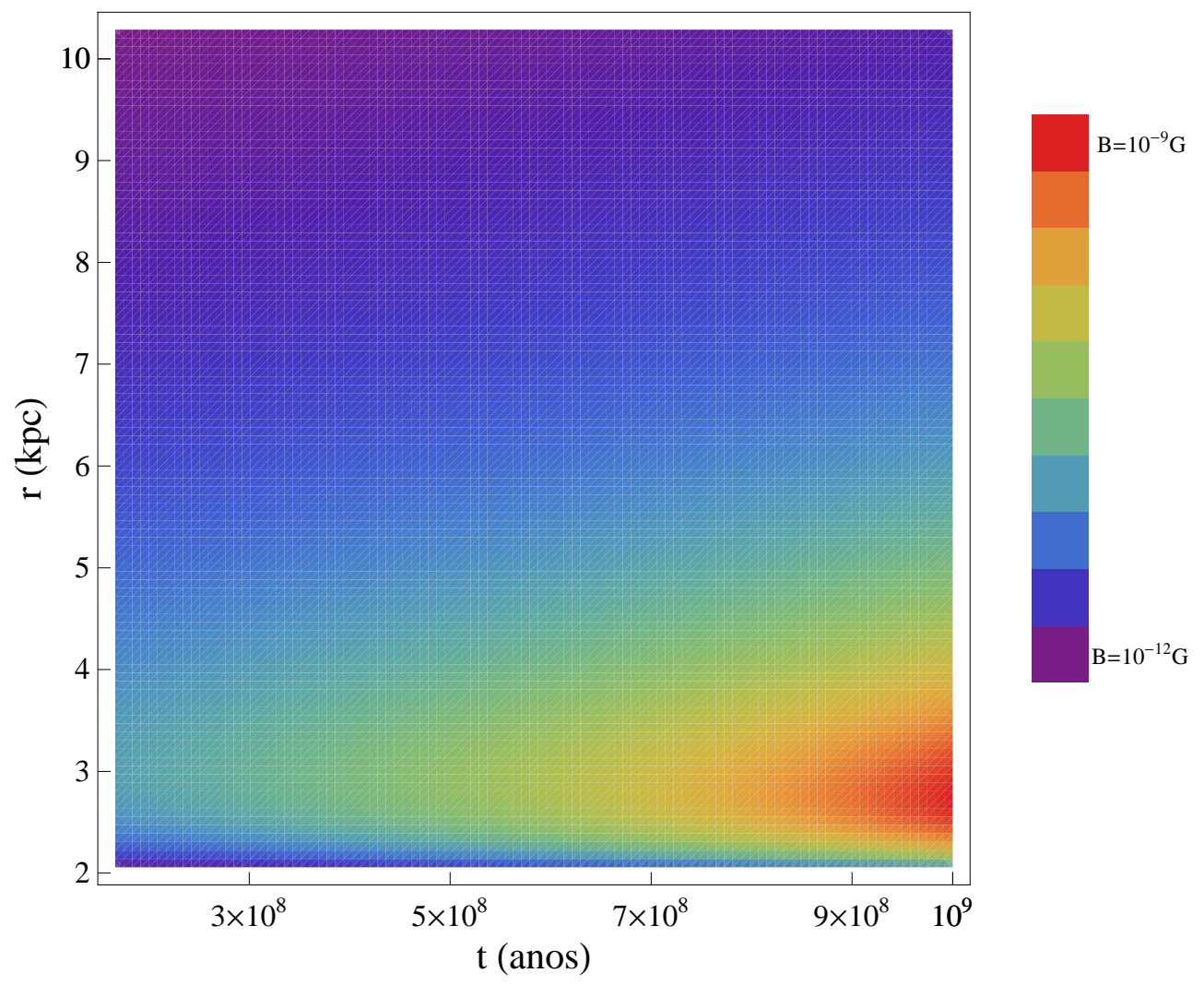

Figura 4.4: Valores do campo magnético $\mathrm{B}(\mathrm{G})$ como função do tempo (anos) e $\mathrm{r}(\mathrm{kpc})$ para os valores de referência (veja fig. 4.2). 
Como podemos ver em nossos cálculos, encontramos campos magnéticos da ordem de $10^{-9}-10^{-8} \mathrm{G}$ em escalas de $1-5 \mathrm{kpc}$, em $z \sim 10$. Da equação (3.51), teremos um campo médio na linha de visada na escala de $200 \mathrm{kpc}$ de $\sim 10^{-9} \mathrm{G}$. Esta escala representa o tamanho da região protogaláctica que irá colapsar gerando as galáxias. Devido a conservação do fluxo magnético, este campo semente será amplificado neste processo pelo fator $\sim\left(L_{P G} / L_{G}\right)^{2} \sim 10^{3}$, onde $L_{P G} \sim 10^{24} \mathrm{~cm}$ é o tamanho típico da protogaláxia e $L_{G} \sim 10^{22} \mathrm{~cm}$, o tamanho da região galáctica após o colapso. Este modelo prevê um campo campo médio observado através de medidas de rotação Faraday com um valor de $\sim 10^{-6}$ G sobre escalas de 5-10 kpc em acordo com os campos magnéticos observados atualmente (Beck 2008; Kulsrud \& Zweibel 2008; Widrow 2002).

\subsection{Considerações}

A origem dos campos magnéticos galácticos pode ser pensada em três principais estágios. Inicialmente temos a formação de um campo magnético fraco, no segundo estágio este campo magnético seria amplificado e por último estes campos seriam modelados e amplificados da forma como são observados hoje em galáxias e protogaláxias.

Neste trabalho consideramos a possibilidade do primeiro estágio ocorrer devido a criação de campos magnéticos no universo primordial, como conseqüência do teorema da flutuação dissipação. Por muito tempo acreditou-se que a amplificação de qualquer campo semente ocorreria devido ao mecanismo de dínamo $\alpha-\Omega$. Contudo, temos várias dificuldades com esta teoria. Inicialmente temos problemas com o congelamento do fluxo magnético que impede que o fluxo total mude de 0 
até um valor finito em uma região finita do plasma. Está condição é satisfeita na teoria padrão $\alpha-\Omega$, pela imposição de condições de contorno, que implicam que durante qualquer amplificação do campo magnético por um valor finito, teríamos a remoção de uma quantidade de fluxo do disco, deixando pra trás um aumento no valor de fluxo de sinal oposto. Este fluxo seria conservado como um todo, mas não na região do disco. Entretanto, este modelo tem grandes dificuldades de ser explicado assumindo campos sementes fracos (Kulsrud \& Zweibel 2008).

O modelo padrão $\alpha-\Omega$, provavelmente não é capaz de explicar a amplificação destes campos sementes em galáxias de alto desvio para o vermelho. Neste caso o passo intermediário seria a amplificação através da turbulência em pequena escala na era protogaláctica. O mecanismo é capaz de amplificar nosso campo semente até as escalas de $\mu \mathrm{G}$ observadas tanto em alto quanto baixo $z$. 


\section{Capítulo 5}

\section{Origem de Campos Magnéticos em Objetos Compactos}

Vários autores já sugeriram uma origem gravitacional para os campos magnéticos em corpos celestes. Estes estudos foram motivados, em parte, pela conjectura de Schuster-Blackett (S-B), onde foi sugerido que o campo magnético presente em planetas e estrelas surgiu devido à sua rotação (Schuster 1980). Neste cenário, uma corrente de massa neutra gera campos magnéticos, implicando na existência de um acoplamento entre o campo magnético e a rotação de um objeto. Uma vez gerado o campo semente através deste mecanismo, outros mecanismos atuariam de forma concomitante, tais como dínamos astrofísicos em geral. Uma das primeiras tentativas no sentido de explorar a conjectura S-B sob a abordagem de uma teoria gravitacional foi feita por Pauli (Pauli 1933). Durante as décadas de 40 e 50, após Blackett ressuscitar a conjectura (Blackett 1947), muitos autores, entre eles Bennett et al. (1949), Papapetrou (1950), Luchak (1952) e Mikhail et al. (1995), tentaram construir uma teoria gravitacional para explicar a relação. Mais 
tarde nos anos 80, Barut \& Gornitz também estudaram a conjectura S-B (Barut \& Gornitz 1985) baseados no formalismo 5-dimensional de Kaluza-Klein (Kaluza 1921; Klein 1926). Este formalismo foi usado com o intuito de descrever uma teoria unificada entre gravitação e eletromagnetismo, onde poderíamos derivar a conjectura S-B a partir de primeiros princípios. Opher \& Wichoski (1997) propuseram que o campo magnético $B \sim 10^{-6}-10^{-5} \mathrm{G}$ em galáxias espirais poderia ser diretamente obtido desta relação. Investigamos neste trabalho a possibilidade desta conjectura poder explicar as observações tanto de magnetares quanto gamma ray bursts.

\subsection{Conjectura Schuster-Blackett}

A conjectura S-B, sugere a geração de campos magnéticos por objetos neutros em rotação. A magnitude do campo é determinada em analogia com o campo magnético gerado por cargas elétricas em rotação. Em particular, Pauli (1933) encontrou um acoplamento anômalo do campo eletromagnético, no qual a razão entre o momento magnético anômalo e o spin de uma partícula elementar era

$$
\frac{\sqrt{G}}{c}
$$

onde devido ao termo extra, ele concluiu que uma massa eletricamente neutra com um spin não nulo precisa ter um pequeno momento magnético. Barut \& Gornitz (1985) sugeriram que em um corpo macroscópico, cada partícula elementar terá um momento magnético anômalo igual ao seu spin $\mathrm{S}$, multiplicado por $\sqrt{G} / c$. Para um objeto macroscópico em rotação, teríamos um acúmulo do momento de 
dipolo magnético $\mathbf{m}$, que poderia ser relacionado ao seu momento angular $\mathbf{L}$ :

$$
\mathbf{m}=\left[\beta \frac{\sqrt{G}}{2 c}\right] \mathbf{L},
$$

onde $\beta$ é uma constante, $G$ a constante de gravitação Newtoniana, e $c$ é a velocidade da luz. O momento angular $\mathbf{L}$ é

$$
\mathbf{L}=I \Omega,
$$

onde $\Omega=2 \pi P^{-1}$ é a velocidade angular, $P$ o período de rotação e $I$ o momento de inércia do objeto. O momento de dipolo magnético $\mathbf{m}$ é relacionado com o campo magnético B por

$$
\mathbf{B}=\frac{3(\mathbf{m} \cdot \mathbf{r}) \mathbf{r}-\mathbf{m r}^{2}}{\mathbf{r}^{5}}
$$

onde $\mathbf{r}$ é a distância de $\mathbf{m}$ até o ponto no qual $\mathbf{B}$ é medido.

Outra possibilidade de obter esta relação de primeiros princípios foi proposta por Mikhail et al. (1995), no contexto de uma teoria generalizada de gravitação,

$$
\begin{aligned}
G_{\mu \nu}+H_{\mu \nu} & =-\kappa T_{\mu \nu}, \\
F_{\mu \nu} & \neq 0
\end{aligned}
$$

onde

$$
H_{\mu \nu}:=\lambda\left[\gamma_{\alpha \beta \mu} \gamma_{\nu}^{\alpha \beta}+\gamma_{\alpha \beta \mu} \gamma_{\nu}^{\alpha \beta}+\gamma_{\alpha \beta \nu} \gamma_{\mu}^{\alpha \beta}+g_{\mu \nu}\left(\gamma_{\alpha \beta \sigma} \gamma^{\sigma \beta \alpha}-\frac{1}{2} \gamma_{\alpha \beta \sigma} \gamma^{\alpha \beta \sigma}\right)\right],
$$


e

$$
F_{\mu \nu}:=\lambda\left[\Phi_{\mu, \nu}-\Phi_{\nu, \mu}-\Phi_{\alpha}\left(\gamma_{\mu \nu}^{\alpha}-\gamma_{\nu \mu}^{\alpha}\right)+\gamma_{\mu \nu}^{\alpha} ; \alpha\right]
$$

Onde recuperamos as equações de campo de Einstein para $\lambda=0$. Eles calcularam as soluções com simetria esférica para esta teoria, no sistema de coordenadas de um objeto em rotação. Para isto, utilizaram a métrica de Reissner-Nordström,

$$
\begin{aligned}
& d s^{2}=g_{\mu \nu} d x^{\mu} d x^{\nu}=-f d t^{2}+\frac{d r^{2}}{f}+r^{2}\left(d \theta^{2}+\sin ^{2} \theta d \varphi^{2}\right) \\
& f(r)=1-\frac{2 M}{r}+\frac{Q^{2}}{r^{2}}
\end{aligned}
$$

As componentes $x^{\mu}$ representam coordenadas espaço-temporais e $\mu, \nu=0,1,2,3$. $M$ e $Q$ são a massa e a carga do objeto respectivamente. Mikhail et al. calcularam as equações de campo para esta métrica, avaliaram o tensor eletromagnético $F^{\mu \nu}$, em um sistema da coordenadas em rotação fazendo $Q=0$. Mesmo tomando como nulo o termo de carga neste sistema de coordenadas em rotação, continuaram encontrando uma contribuição para o campo magnético como efeito puramente devido à rotação do objeto, dada por:

$$
B_{p}=\frac{9}{4} \sqrt{\frac{2 M}{R}} \Omega \cos (\theta),
$$

onde $B_{p}$ é o campo magnético gerado pelo objeto, $M$ a sua massa, $R$ o raio, $\Omega$ sua velocidade angular e $\theta$ o ângulo entre o dipolo magnético gerado e o vetor momento angular do corpo.

Sirag (1979) comparou as previsões da conjectura S-B para objetos do sistema solar: Terra, Sol, Lua, Mercúrio, Vênus, Júpiter, Saturno; para a estrela 78 Vir e a 
estrela de nêutrons Her X-1, como podemos ver na figura 5.1. Ele encontrou valores para o parâmetro $\beta$ da equação (5.4) entre 0.02-0.77, com exceção da estrela 78 Vir como podemos ver na tabela (5.1). Woodward (1989) examinou a conjectura com pulsares de curto período. Eles acharam que $\beta$ não é o mesmo para todos os pulsares. Pulsares jovens mantém seu valor individual de $\beta$ constante apenas por um certo período de tempo. Eles acharam valores de $\beta$ para os pulsares na faixa de 0.001 to 0.01 .

\begin{tabular}{|c|c|c|c|c|c|c|c|c|c|}
\hline $\begin{array}{l}\text { Body } \\
\text { (refs) }\end{array}$ & $f$ & $\begin{array}{c}\stackrel{m}{\text { Mass }} \\
(\mathrm{kg})\end{array}$ & $\begin{array}{c}\begin{array}{c}r \\
\text { Radius }\end{array} \\
\text { (m) }\end{array}$ & $\begin{array}{c}\omega \\
\text { Angular } \\
\text { velocity }\end{array}$ & $\begin{array}{c}B \\
\begin{array}{c}\text { Magnetic } \\
\text { field }\end{array} \\
\text { (T) }\end{array}$ & $\begin{array}{c}U \\
\text { Angular } \\
\text { momentum } \\
\frac{2}{5} \cdot f \omega \mathrm{m} r^{2} \\
(\mathrm{~J} \mathrm{~s})\end{array}$ & $\begin{array}{c}P \\
\text { Magnetic } \\
\text { moment } \\
\frac{1}{2} \cdot \frac{4 \pi}{\mu_{0}} \cdot \mathrm{Br}^{3} \\
\left(\mathrm{~A} \mathrm{~m}^{2}\right)\end{array}$ & $\begin{array}{c}P / U \\
\times 10^{-11}\end{array}$ & $\frac{P}{U} \div \frac{G^{\dagger}}{2 k^{\frac{1}{2}}}$ \\
\hline $\begin{array}{l}\text { Earth } \\
\qquad(1,15-17)\end{array}$ & 0.88 & $5.98 \times 10^{24}$ & $6.378 \times 10^{6}$ & $7.272 \times 10^{-5}$ & $\begin{array}{l}7.0 \times 10^{-5} \\
2.5 \times 10^{-5}\end{array}$ & $6.22 \times 10^{33}$ & $\begin{array}{l}9.1 \times 10^{22} \\
3.2 \times 10^{22}\end{array}$ & $\begin{array}{l}1.5 \\
0.51\end{array}$ & $\begin{array}{l}0.35 \\
0.12\end{array}$ \\
\hline $\begin{array}{l}\text { Sun } \\
\qquad(15,16,18,19)\end{array}$ & 0.16 & $1.99 \times 10^{30}$ & $6.960 \times 10^{8}$ & $\begin{array}{l}2.9 \times 10^{-6} \\
2.7\end{array}$ & $\begin{array}{l}27 \times 10^{-4} \\
0.8 \times 10^{-4}\end{array}$ & $\begin{array}{c}1.79 \times 10^{41} \\
1.67 \times 10^{41}\end{array}$ & $\begin{array}{l}4.6 \times 10^{30} \\
0.14 \times 10^{30}\end{array}$ & $\begin{array}{l}2.7 \\
0.078\end{array}$ & $\begin{array}{c}0.63 \\
0.02\end{array}$ \\
\hline $\begin{array}{l}78 \mathrm{Vir} \\
(1-3)\end{array}$ & 0.16 & $\begin{array}{l}4.8 \times 10^{30} \\
4.4 \times 10^{30}\end{array}$ & $\begin{array}{l}1.8 \times 10^{9} \\
1.0 \times 10^{9}\end{array}$ & $\begin{array}{r}5 \times 10^{-4} \\
0.5 \times 10^{-4}\end{array}$ & $\begin{array}{l}0.2 \\
0.1\end{array}$ & $\begin{array}{r}5.0 \times 10^{44} \\
0.14 \times 10^{44}\end{array}$ & $\begin{array}{l}5.8 \times 10^{33} \\
0.5 \times 10^{33}\end{array}$ & $\begin{array}{c}41 \\
0.1\end{array}$ & $\begin{array}{l}9.8 \\
0.02\end{array}$ \\
\hline $\begin{array}{l}\text { Moon } \\
\qquad(3,15,16)\end{array}$ & 0.88 & $7.35 \times 10^{22}$ & $1.738 \times 10^{6}$ & $2.66 \times 10^{-6}$ & $\begin{array}{r}10.3 \times 10^{-8} \\
3.8 \times 10^{-8}\end{array}$ & $2.08 \times 10^{29}$ & $\begin{array}{l}2.7 \times 10^{18} \\
1.0 \times 10^{18}\end{array}$ & $\begin{array}{l}1.3 \\
0.48\end{array}$ & $\begin{array}{l}0.30 \\
0.11\end{array}$ \\
\hline $\begin{array}{l}\text { Mercury } \\
\qquad(4,6,15)\end{array}$ & 0.88 & $3.3 \times 10^{23}$ & $2.434 \times 10^{6}$ & $1.24 \times 10^{-6}$ & $\begin{array}{l}4 \times 10^{-7} \\
2 \times 10^{-7}\end{array}$ & $8.5 \times 10^{29}$ & $\begin{array}{l}2.8 \times 10^{19} \\
1.4 \times 10^{19}\end{array}$ & $\begin{array}{l}3.3 \\
1.6\end{array}$ & $\begin{array}{l}0.77 \\
0.37\end{array}$ \\
\hline $\begin{array}{l}\text { Venus } \\
\qquad(6,15)\end{array}$ & 0.88 & $4.87 \times 10^{24}$ & $6.052 \times 10^{6}$ & $2.99 \times 10^{-7}$ & $\begin{array}{l}5.9 \times 10^{-8} \\
2.7 \times 10^{-8}\end{array}$ & $1.88 \times 10^{31}$ & $\begin{array}{r}6.5 \times 10^{19} \\
3 \times 10^{19}\end{array}$ & $\begin{array}{l}0.35 \\
0.16\end{array}$ & $\begin{array}{l}0.08 \\
0.04\end{array}$ \\
\hline $\begin{array}{l}\text { Jupiter } \\
\qquad(5,6,15)\end{array}$ & 0.66 & $1.90 \times 10^{27}$ & $7.143 \times 10^{-7}$ & $1.773 \times 10^{-4}$ & $\begin{array}{r}14 \times 10^{-4} \\
3 \times 10^{-4}\end{array}$ & $4.54 \times 10^{38}$ & $\begin{array}{r}26 \times 10^{26} \\
5.5 \times 10^{26}\end{array}$ & $\begin{array}{l}0.57 \\
0.12\end{array}$ & $\begin{array}{l}0.13 \\
0.03\end{array}$ \\
\hline $\begin{array}{l}\text { Saturn } \\
\qquad(6,15,20)\end{array}$ & 0.66 & $5.69 \times 10^{26}$ & $5.98 \times 10^{7}$ & $1.706 \times 10^{-4}$ & $\begin{array}{l}3 \times 10^{-4} \\
1 \times 10^{-4}\end{array}$ & $9.16 \times 10^{37}$ & $\begin{array}{l}3.2 \times 10^{26} \\
1.2 \times 10^{26}\end{array}$ & $\begin{array}{l}0.35 \\
0.13\end{array}$ & $\begin{array}{l}0.08 \\
0.03\end{array}$ \\
\hline $\begin{array}{l}\text { Her } X-1^{*} \\
\quad(7,8,21,22)\end{array}$ & 0.88 & $3.5 \times 10^{30}$ & $\begin{array}{r}1 \times 10^{4} \\
0.7 \times 10^{4}\end{array}$ & 5.076 & $\begin{array}{l}7 \times 10^{8} \\
5 \times 10^{8}\end{array}$ & $\begin{array}{l}3.1 \times 10^{38} \\
1.8 \times 10^{38}\end{array}$ & $\begin{array}{r}35 \times 10^{25} \\
8.6 \times 10^{26}\end{array}$ & $\begin{array}{l}1.9 \\
0.28\end{array}$ & $\begin{array}{l}0.44 \\
0.07\end{array}$ \\
\hline
\end{tabular}

Tabela 5.1: Tabela extraída de Sirag (1979), onde nós temos os dados da razão máxima e mínima do momento angular e magnético para corpos celestes.

Nas próximas seções faremos uma análise destas relações, como um possível candidato para origem dos campos magnéticos em magnetares e GRBs. 


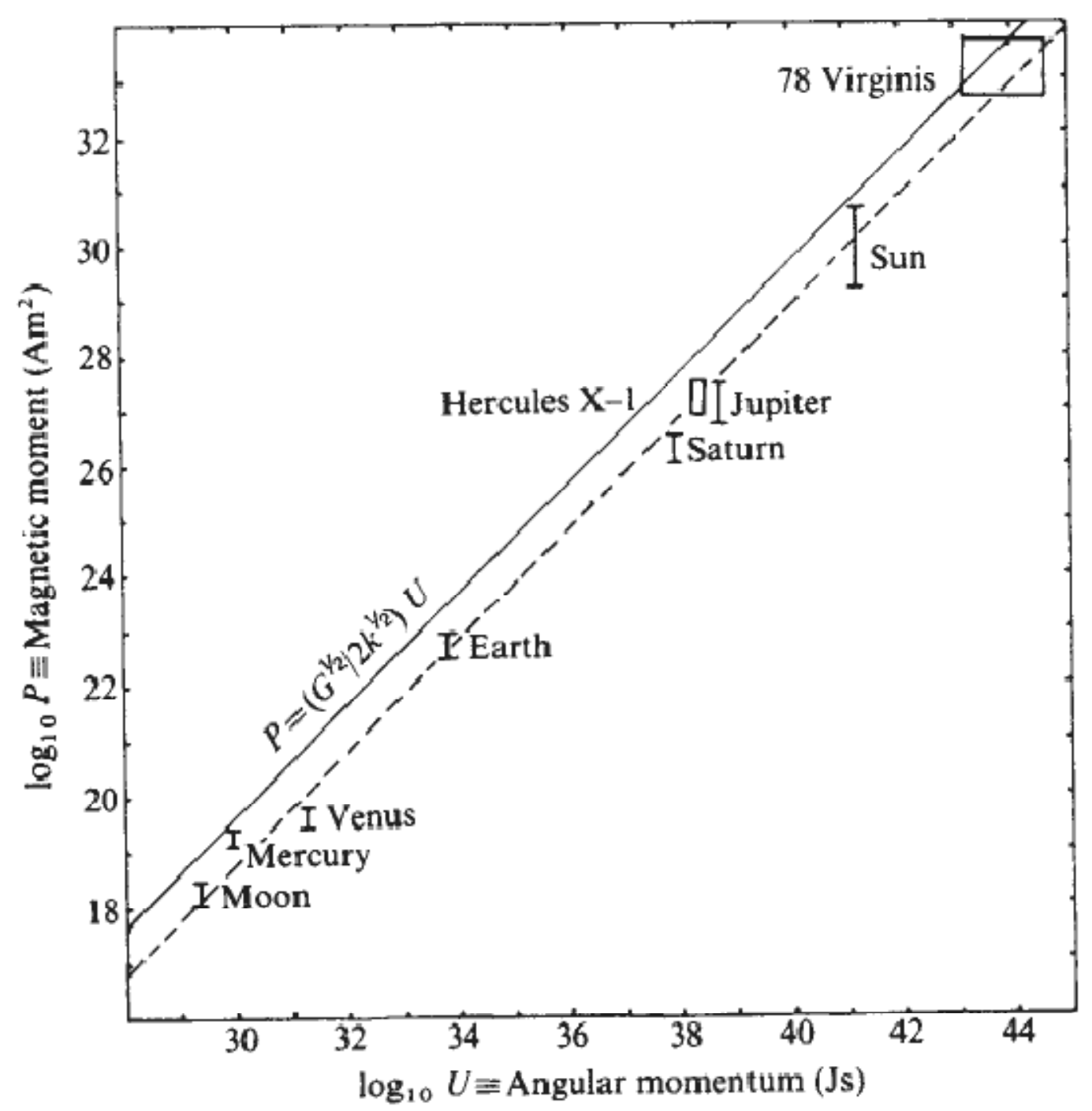

Figura 5.1: Gráfico extraído de Sirag (1979), onde temos um diagrama da razão momento magnético $P$ e momento angular $U$. Temos a linha contínua representando a previsão da conjectura $S-B, P=\left(G^{1 / 2} / 2 k^{1 / 2}\right) U$. A linha pontilhada representa a regressão linear para os dados dos objetos. 


\subsection{Magnetares}

Magnetares são estrelas de nêutrons cujo valor do campo magnético é maior que o valor quântico crítico, $B_{Q E D}=4.4 \times 10^{13} G$, onde os níveis de energia dos elétrons se iguala à sua massa de repouso. Seus campos magnéticos têm intensidades típicas de 100-1000 vezes maiores que uma estrela de nêutrons típica, como os rádio pulsares. Juntos com outras classes de estrelas de nêutrons observadas em todo espectro, eles indicam que os clássicos rádio pulsares descobertos inicialmente eram apenas uma das diversas manifestações das estrelas de nêutrons. O modelo de magnetar foi introduzido por Duncan \& Thompson (1992); Thompson \& Duncan (1995). Podemos dividir os magnetares em duas classes que foram descobertas de forma independente, os Anomalous X-ray pulsars (AXPs) e os Soft Gamma-ray Repeaters (SGRs).

SGRs foram inicialmente descobertos através da detecção de surtos curtos, na faixa dos raios-X duros e raios- $\gamma$ moles. Foram inicialmente considerados uma subclasse de gamma-ray bursts (Laroes et al. 1986; Atteia et al. 1987). AXPs foram inicialmente detectados na faixa dos raios-X moles $(<10 \mathrm{keV})$, suas propriedades peculiares fizeram com que fossem classificados como uma classe diferente de pulsares Mereghetti \& Stella (1995). Observações indicam certas similaridades entre estes dois objetos. Então o modelo de magnetar inicialmente desenvolvido para explicar as propriedades dos SGRs, foi também aplicado aos AXPs (Thompson \& Duncan 1996).

AXPs possuem um espectro em raios-X não Planckiano, sendo em geral descrito por uma função empírica tipo corpo negro $\left(k_{B} T \sim 0.3-0.6 k e V\right)$ mais uma lei de potência. Algumas das características dos AXPs são a falta de evidência de uma 
companheira, possuem períodos de rotação na faixa de 5-15 segundos, espectro em raios-X moles e ausência de emissão em rádio e uma taxa de variação do seu período $\dot{P} \sim 10^{-11} s^{-1}$. Os SGRs possuem surtos de radiação- $\gamma$ mole $\sim 100 \mathrm{~ms}$, possuem períodos de rotação na faixa de $5-8$ segundos, com $\dot{P} \sim 10^{-10} s s^{-1}$. Atualmente conhecemos 16 magnetares, dos quais 6 SGRs (4 confirmados e 2 candidatos) e 10 AXPs (9 confirmados e 1 candidato), como podemos ver nas tabelas (5.3) e (5.2).

Podemos argumentar que estes objetos possuem altos campos magnéticos analisando a taxa de decaimento de sua rotação. Assumindo que este decaimento ocorre devido à perda de energia gerada pela radiação de dipolo magnético no vácuo, a intensidade deste campo pode ser estimada por

$$
B_{\text {dip }}=2.48 \times 10^{14}(P / 6 s)^{1 / 2}\left(\dot{P} / 10^{11} s s^{-1}\right)^{1 / 2} G \text {. }
$$

Para uma estrela de nêutrons, o momento de inércia característico é $I=10^{45} \mathrm{gcm}^{2}$ e seu raio é de $R=10 \mathrm{~km}$. Neste contexto, o campo magnético associado com os AXPs excede $B \gtrsim 5 \times 10^{13} \mathrm{G}$.

A formação dos magnetares, em especial a origem dos seus altos campos magnéticos permanece um problema em aberto na astrofísica. Os modelos pra sua origem podem ser divididos em dois grandes cenários. Podemos considerar que estes campos magnéticos foram gerados por algum mecanismo de dínamo ou assumir que são campos fósseis já existentes nos progenitores destes magnetares.

Duncan \& Thompson (1992) exploraram a amplificação turbulenta destes campos magnéticos na zona convectiva do progenitor destas estrelas, assim como devido a rotação diferencial das estrelas de nêutrons na época do seu nascimento, concluindo que em princípio campos magnéticos de até $\sim 3 \times 10^{17} \mathrm{G}$ poderiam 
ser criados. Entretanto este mecanismo necessita que a estrela de nêutrons no momento da sua criação tenha uma rotação extremamente rápida com período de alguns milisegundos. E a população observada de magnetares possui períodos na faixa de $\sim 2-12$. Logo este cenário tem problemas em explicar o fato dos magnetares terem períodos maiores do que o previsto.

Outro cenário proposto sugere um campo magnético fóssil. Eles consideram que estes campos podem ter vindo de estrelas $\mathrm{O}$ e B, com altos campos magnéticos $\left(\sim 10^{4} \mathrm{G}\right)$. Durante a sua transformação em estrelas de nêutrons, este campo inicial seria amplificado até os valores observados nos magnetares (Vink \& Kuiper 2006). Contudo, isto só transfere o problema, pois precisamos explicar os altos campos magnéticos na estrela progenitora.

Iremos analisar a previsão da conjectura S-B para estes objetos, de forma a considerar uma hipótese alternativa para origem de tais campos. Podemos escrever o campo magnético de uma estrela de nêutrons previsto pela conjectura S-B da forma

$$
\begin{aligned}
B_{S-B} & =\beta c^{-1} G^{1 / 2} I r^{-3} 2 \pi P^{-1} G \\
& \simeq 5.414 \times 10^{13} \beta P^{-1} G .
\end{aligned}
$$

Se quisermos associar as equações (5.4) e (5.9), basta consideramos $\beta$ não mais uma constante, mas sim proporcional ao potencial gravitacional $\phi=2 M / R$ do objeto

$$
\beta=\frac{45 c}{8 G^{1 / 2} \phi^{1 / 2}}
$$

Devemos comparar os campos magnéticos previstos pelas expressão (5.10) com 
previsões das equações (5.4), e (5.9). Para isto, utilizamos dados da literatura dos períodos dos AXPs e $\mathrm{SGRs}^{1}$. Calculamos o valor do parâmetro $\beta$ para equação (5.4) e de $\theta$ em (5.9) utilizando a função de verossimilhança $\mathcal{L} \propto \exp \left(-\chi^{2} / 2\right)$, onde

$$
\begin{array}{r}
\chi_{1}^{2}(\beta)=\sum_{i=1}^{N} \frac{\left[B_{d i p}^{i}(P, \dot{P})-B_{S-B}^{i}(P, \beta)\right]^{2}}{\sigma_{i}^{2}}, \\
\chi_{2}^{2}(\theta)=\sum_{i=1}^{N} \frac{\left[B_{d i p}^{i}(P, \dot{P})-B_{p}^{i}(P, \theta)\right]^{2}}{\sigma_{i}^{2}}
\end{array}
$$

onde $\mathrm{N}$ é o número de magnetares da amostra.

Nas figs. 5.2 e 5.3 mostramos a distribuição de probabilidade dos parâmetros $\beta$ e $\theta$ para a nossa amostra. Como podemos observar, o melhor ajuste do modelo gera $\beta \sim 17 \pm 13.64$ e $\theta \sim 0 \pm 0.3 \pi$. Podemos concluir comparando o valor de $\beta$ encontrado que, apesar de haver uma correlação entre o momento angular e o momento magnéticos de objetos celestes em diversas escalas, a relação (5.4) não ajusta todas as observações com um $\beta$ constante. Podemos imaginar duas possibilidades para esta discrepância, no caso da conjectura S-B ser verdadeira. A primeira é o fato de haver outros mecanismos concomitantes que não permitiriam uma medida exata apenas do campo magnético gerado pela conjectura S-B, como por exemplo efeitos de dínamo. A segunda opção é que $\beta$ não seja uma constante, e sim dependa do potencial gravitacional do objeto, como sugeriu Mikhail et al. (1995)

Como podemos ver na fig. (5.3), o modelo para $\beta$ proporcional ao potencial gravitacional do objeto, se ajusta muito bem aos dados, tendo como melhor ajuste

\footnotetext{
${ }^{1}$ http://www.physics.mcgill.ca/ pulsar/magnetar/main.html
} 
um alinhamento do momento magnético próximo ao momento angular do magnetar.

Os campos magnéticos intensos $\sim 10^{15} \mathrm{G}$, observados nos magnetares não são facilmente produzidos por mecanismos usuais. Nós examinamos a possibilidade deles serem produzidos por algum tipo de acoplamento entre o campo gravitacional e eletromagnético dos objetos celestes. Obtivemos valores em acordo com os observados considerando o modelo de $\beta$ variável. Isto indica que se a conjectura SB estiver correta, poderíamos ter uma explicação simples para origem dos campos magnéticos em magnetares. 
CAPÍTULO 5. ORIGEM DE CAMPOS MAGNÉTICOS EM OBJETOS COMPACTOS

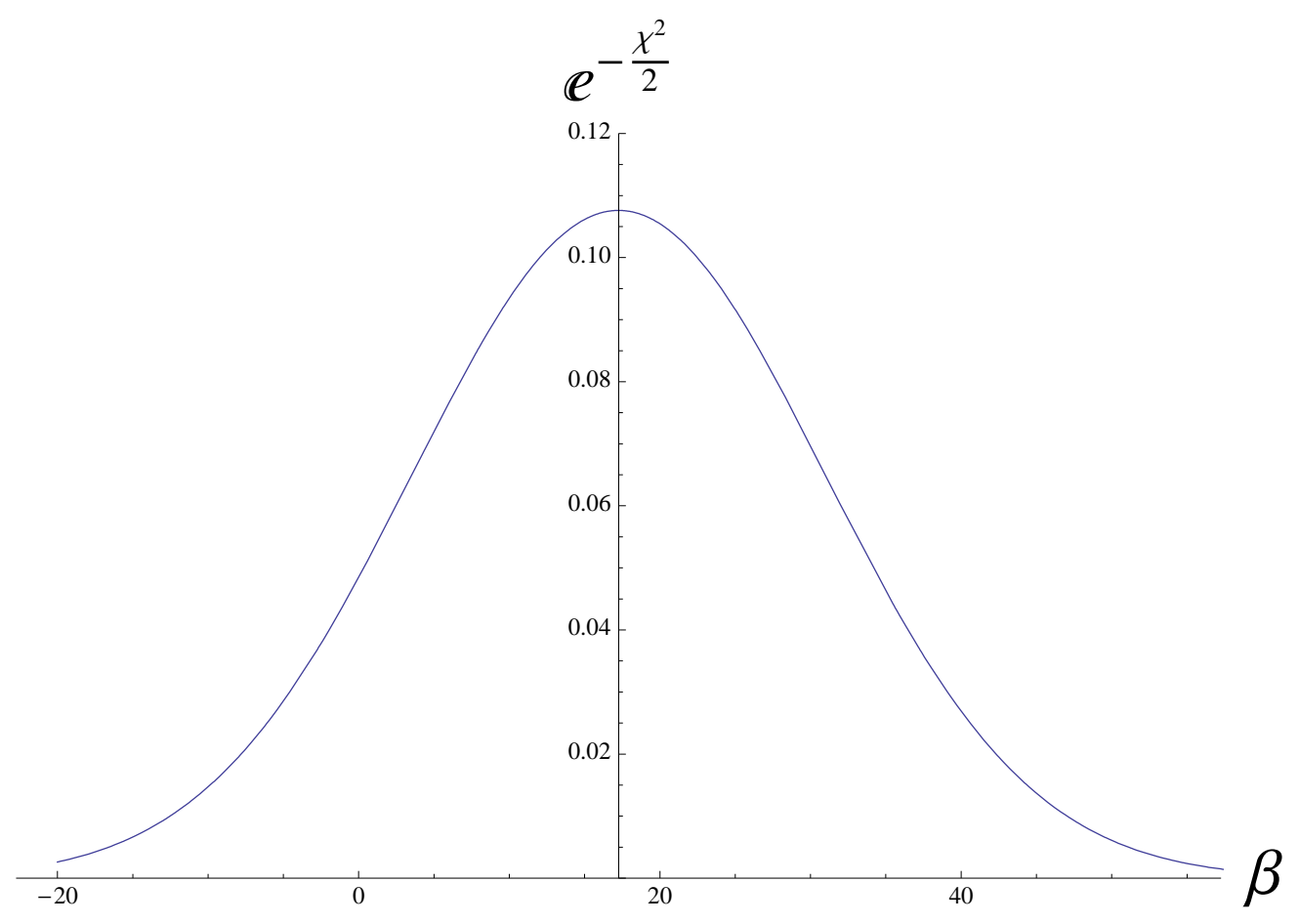

Figura 5.2: Distribuição de verossimilhança do parâmetro $\beta$ para os dados de AXPs e SGRs. 


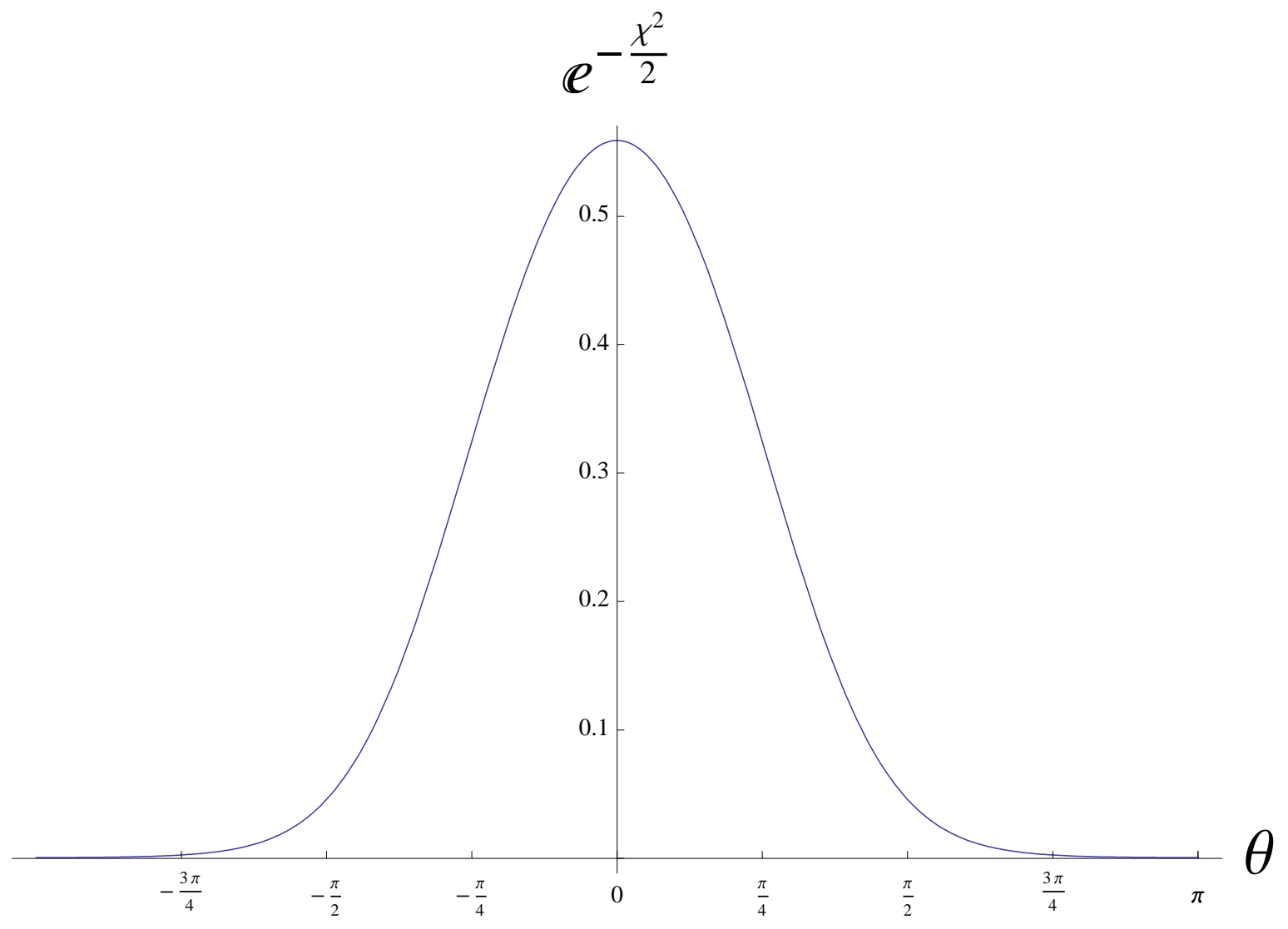

Figura 5.3: Distribuição de verossimilhança do parâmetro $\theta$ para os dados de AXPs e SGRs. 
Tabela 5.2: Soft Gamma-ray Repeaters. Col(1): Nome dos SGRs; Col(2): Período dos SGRs; $\mathrm{Col}(3)$ : Spindown dos SGRs.

\begin{tabular}{|l|c|c|}
\hline Nome & $\mathrm{P}(\mathrm{s})$ & $\dot{P}\left(s^{-1}\right)$ \\
\hline SGR 1900+14 & $5.16891778(21)$ & $7.783(8)$ \\
SGR 1627-41 & $6.41318(3)$ & $* *$ \\
SGR 1806-20 & $7.55592(5)$ & $54.9(9)$ \\
SGR 0526-66 & $8.0470(2)$ & $6.5(5)$ \\
SGR 1801-23 & $* *$ & $* *$ \\
SGR 0501+4516 & $5.7620699(4)$ & $0.5(1)$ \\
\hline
\end{tabular}

Tabela 5.3: Anomalous X-ray pulsars. $\mathrm{Col}(1)$ : Nome dos AXPs; Col(2): Período dos AXPs; Col(3): Spindown dos AXPs.

\begin{tabular}{|l|c|c|}
\hline Nome & $\mathrm{P}(\mathrm{s})$ & $\dot{P}\left(s s^{-1}\right)$ \\
\hline 1E 1547.0-5408 & $2.06983302(4)$ & $2.318(5)$ \\
XTE J1810-197 & $5.539425(16)$ & $0.81(7)$ \\
1E 1048.1-5937 & $6.45207658(54))$ & 2.70 \\
AX J1845-0258 & $6.97127(28)$ & $* *$ \\
1E 2259+586 & $6.9789484460(39)$ & $0.048430(8)$ \\
CXOU J010043.1-721134 & $8.020392(9)$ & $1.88(8)$ \\
4U 0142+61 & $8.68832973(8)$ & $0.1960(2)$ \\
CXO J164710.2-455216 & $10.6107(1)$ & $0.24(6)$ \\
1RXS J170849.0-400910 & $10.9990355(6)$ & $1.945(2)$ \\
1E 1841-045 & $11.7750542(1)$ & $4.1551(14)$ \\
\hline
\end{tabular}




\subsection{Gamma Ray Bursts}

Gamma ray bursts (GRBs) são as mais concentradas e brilhantes explosões eletromagnéticas do Universo, gerando pulsos intensos de raios- $\gamma$. Os surtos duram de uma fração até centenas de segundos. Os GRBs podem chegar de distâncias cosmológicas e direções aleatórias do céu. Possuem luminosidades da ordem de $10^{51}-10^{53} \mathrm{ergss}^{-1}$. Os candidatos para a classe de surtos longos de raios- $\gamma$ são em geral estrelas massivas colapsando em buracos negros, enquanto os surtos curtos, têm como possível progenitor a coalescência de estrelas de nêutrons binárias ou um sistema binário com uma estrela de nêutrons e um buraco negro (Meszaros 2006). Investigamos o cenário onde GRBs consistem em um buraco negro com um campo magnético ao redor. A fonte de energia neste caso seria dada pela rotação do buraco negro que seria extraída através do fluxo de Poyting (Piran 2005) .

A energia necessária para gerar os GRBs $10^{51}-10^{53} \mathrm{ergss}^{-1}$ pode ser compreendia de forma mais clara em comparação com a energia associada à massa de repouso do Sol, da ordem de $2 \times 10^{54}$ ergs. Ficando evidente que um bom modelo para região interna do GRB precisa ser capaz de extrair uma quantidade significativa de energia de sua fonte progenitora, sendo ela uma estrela de nêutrons ou um buraco negro. Outro critério é a necessidade de poder extrair energia da fonte por um período de pelo menos $\sim 1000 \mathrm{~s}$ de forma a explicar os surtos de longa duração (Lee et al. 2000).

A quantidade de energia que pode ser extraída de um buraco negro, sem violar a segunda lei da termodinâmica, é da ordem de sua energia de rotação dada por

$$
E_{\text {rot }}=M c^{2}-M_{i r r} c^{2},
$$


onde

$$
M_{i r r}=\sqrt{\frac{S_{B N}}{4 \pi k_{B}}} M_{\text {Planck }}
$$

$M_{i r r}$ é a massa irredutível do buraco negro, $S_{B N^{2}}$ a sua entropia e $M_{\text {Planck }}{ }^{3}$ é a massa de Planck. A energia de rotação de um buraco negro com momento angular $J$ é uma fração de sua massa $M$,

$$
\begin{aligned}
E_{\text {rot }} & =f(\alpha) M c^{2} \\
f(\alpha) & =1-\sqrt{\frac{1}{2}\left[1+\sqrt{1-\alpha^{2}}\right]},
\end{aligned}
$$

onde $\alpha=J c / M^{2} G$ é o parâmetro de rotação. Para um buraco negro com rotação máxima $(\alpha=1), f=0.294$. Para o caso em que a extração é máxima podemos escrever a potência do fluxo de Poyting emitida pelo buraco negro da forma

$$
P_{B Z}=6.7 \times 10^{50}\left(\frac{B}{10^{15}}\right)^{2}\left(\frac{M}{M_{\odot}}\right)^{2} \operatorname{ergss}^{-1}
$$

(Lee et al. 2000). Se a forma de transporte de energia for feita através de um fluxo de Poynting, a escala de tempo deste processo pode ser calculada como a razão entre a massa do buraco negro e potência transportada através da superfície do buraco negro $\propto R^{2} c B^{2}$,

$$
\begin{aligned}
\tau_{B Z} & \sim \frac{M c^{2}}{B^{2} R^{2} c} \\
& =2.7 \times 10^{3}\left(\frac{10^{15}}{B}\right)^{2}\left(\frac{M_{\odot}}{M}\right) s
\end{aligned}
$$

onde $M$ é a massa do buraco negro, $R$ é o raio do horizonte $\sim G M / c^{2}$, e $B$ o

\footnotetext{
${ }^{2} S_{B N}=A_{B H} k_{B} c^{3} / 4 G \hbar$, onde $A_{B H}$ é a área superficial do buraco negro.

${ }^{3} M_{\text {Planck }}=\sqrt{c \hbar / G}$
} 
campo magnético no horizonte.

Para analisar a previsão da conjectura S-B nos GRBs, precisamos de uma estimativa do parâmetro de rotação do buraco negro central. Uma estimativa razoável é considerar o parâmetro de rotação dos buracos negros seja da ordem da 0.5-1.0 (Popham et al. 1999).

Usando as equações. (5.4) e (5.9), o campo magnético na vizinhança do buraco negro pode ser escrito como

$$
\begin{aligned}
B_{S-B} & =\frac{G^{3 / 2} M^{2} \alpha \beta}{c^{2} R^{3}} \approx 225\left(\frac{M}{M_{\odot}}\right)^{2}\left(\frac{R}{R_{\odot}}\right)^{3} \alpha \beta G, \\
B_{p} & =\frac{9}{4} M \sqrt{\frac{5 G \alpha}{R^{3} c}} \approx 8.13 \times 10^{8}\left(\frac{M}{M_{\odot}}\right) \alpha^{1 / 2}\left(\frac{R}{R_{\odot}}\right)^{-3 / 2} G .
\end{aligned}
$$

Podemos calcular a previsão das equações (5.4) e (5.9) utilizando alguns valores característicos. Segundo Lee et al. (2000), esperamos que $\alpha \sim 0.1-1$ e M $\sim 2.5 M_{\odot}$. O raio de um buraco negro de Kerr é dado por

$$
R_{B N}=\frac{R_{S h}}{2}\left[1+\sqrt{1-\alpha^{2}}\right]
$$

onde $R_{S h}=2 G M / c^{2}$ é o raio de Schwarzschild.

A previsão dada por (5.4), considerando $\beta \sim 0.01-0.1$ é $B_{S-B} \sim 10^{15}-10^{16}$ G. Enquanto a previsão da equação (5.9) para os mesmos parâmetros é $B_{p} \sim 10^{16}$ G. Utilizando as expressões (5.19) e (5.20) com estes valores de campo magnético, podemos concluir que a intensidade dos mesmos é capaz de gerar uma energia da ordem de $10^{51}-10^{53}$ ergs. Podendo suprir o GRBS por intervalos de tempo de até $2.3 \times 10^{3} \mathrm{~s}$. 


\subsection{Considerações}

Observações indicam a presença de intensos campos magnéticos em GRBs e magnetares. Teorias astrofísicas usuais têm grande dificuldade em explicar tais campos. Neste trabalho avaliamos a previsão oriunda da conjectura S-B para estes objetos, como uma explicação alternativa para estes campos.

Em GRBs, a existência de campos magnéticos de $\sim 10^{15} \mathrm{G}$ poderia explicar o fluxo de Poynting requerido para suprir a energia necessária aos surtos observados. Contudo não há um bom modelo para explicar a existência destes campos, se consideramos a conjectura S-B verdadeira, podemos explicar tais campos na região do horizonte do buraco negro.

Da mesma forma avaliamos os valores a previsão da conjectura S-B em estrelas de nêutrons com altos campos magnéticos denominadas magnetares. Não é fácil produzir intensos campos magnéticos nestes objetos. Mostramos aqui, que se a conjectura S-B é verdadeira, poderíamos explicar naturalmente a origem destes campos. 


\section{Capítulo 6}

\section{Efeitos de Campos Magnéticos e}

\section{Turbulência em Aglomerados de}

\section{Galáxias}

Os aglomerados de galáxias são as maiores estruturas virializadas (ou próximas do estado de equilíbrio) no Universo. Dentro do cenário hierárquico de formação de estruturas, eles teriam se formado em $z \lesssim 1$. Aglomerados são poderosas ferramentas, sendo capazes de vincular os valores da matéria e energia escura. Muitos estudos necessitam do valor da massa total do aglomerado, entretanto este valor é difícil de ser medido com acurácia. Dados em raios-X são freqüentemente usados na determinação da distribuição da massa de aglomerados de galáxias. Neste método, o equilíbrio hidrostático é em geral assumido e as observações da densidade e temperatura do gás intraglomerado são utilizadas para inferir sua pressão térmica, de modo a avaliar sua massa dinâmica (e.g., David et al. 1995; White \& Fabian 1995; Finoguenov et al. 2001; Reiprich \& Böhringer 2002). 
O método de determinação de massa através de medidas de raios-X utiliza os perfis tanto da densidade do gás quanto da temperatura do meio intraglomerado. Com estes dados podemos resolver a equação do equilíbrio hidrostático para obtenção do perfil da massa total assumindo simetria esférica. Outros métodos muito utilizados são a estimativa da massa de virial através de medidas de dispersão de velocidades ou através de medidas de lentes gravitacionais.

Em geral apenas a pressão térmica do gás é considerada na equação de equilíbrio hidrostático. Neste trabalho incluímos os efeitos das pressões não térmicas $P_{\mathrm{NT}}$, compostas pela pressão magnética $\left(P_{\mathrm{B}}\right)$ e pressão turbulenta $\left(P_{\text {turb }}\right)$ na determinação da massa de aglomerados.

Apesar da dificuldade em calcular acuradamente as propriedades do campo magnético na região intraglomerado, a existência deles é bem estabelecida por estudos de rotação Faraday e emissão síncrotron de fontes difusas (e.g., Andernach et al. 1988; Giovannini et al. 1993; Taylor et al. 1994, 2002; Govoni \& Feretti 2004). Campos magnéticos intensos podem contribuir significativamente ao suporte de pressão do gás (Loeb \& Mao 1994), contribuindo como um componente não térmico na equação de equilíbrio hidrostático (Dolag et al. 2001b). De fato, campos magnéticos da ordem de 10-100 $\mu \mathrm{G}$ foram achados nos aglomerados de Hydra A (Taylor et al. 1993), Cygnus A (Dreher et al. 1987) e 3C 295 (Perley \& Taylor 1991).

Testes utilizando simulações cosmológicas em aglomerados mostram a presença de fluxos subsônicos no gás, mesmo em aglomerados relaxados (Lau et al. 2009). Estes movimentos podem ser gerados pela contínua acresção de gás em aglomerados ao longo de filamentos, devido a mergers ou movimentos de galáxias através do meio intraglomerado. Ondas de choque podem gerar turbulência em escalas 
comparáveis ao tamanho do aglomerado (Lau et al. 2009). Estes grandes vórtices podem transferir energia em cascata para escalas menores gerando um espectro de turbulência no aglomerado. Em escalas menores a turbulência pode ser gerada pelo movimento de galáxias, assim como pelos jatos oriundos de núcleos ativos de galáxias. Análises de aglomerados simulados mostram que $\approx 10 \%-20 \%$ do suporte de pressão em aglomerados vêm de movimentos subsônicos do gás (Rasia et al. 2004, 2006). Vários estudos nos últimos anos, têm considerado os efeitos tanto da pressão magnética quanto da pressão turbulenta em aglomerados simulados (Dolag et al. 2001a; Colafrancesco \& Giordano 2007; Dolag et al. 2005; Rasia et al. 2004, 2006, entre outros). Nosso objetivo neste trabalho é quantificar estes dois efeitos em aglomerados reais. Para isto, utilizamos dados de cinco aglomerados de Abell observados pelo XMM-Newton'1 : A496, A2050, A1689, A2667 e A2631. Em nossa análise, utilizamos os perfis de temperatura e densidade de Laganá et al. (2008) e introduzimos a contribuição das pressões não térmicas $P_{\mathrm{NT}}$ na equação de equilíbrio hidrostático.

\subsection{Dados da Amostra}

Os dados referentes aos cinco aglomerados de Abell utilizados na análise, estão disponíveis no arquivo público do XMM-Newton na faixa de desvio para o vermelho $0.03<z<0.3$. Estes aglomerados foram previamente analisados por Laganá et al. (2008), que derivaram os parâmetros dos seus perfis de densidade para inferência de suas massas.

Apesar de não ter sido utilizado nenhum critério de seleção morfológica para

\footnotetext{
${ }^{1}$ http://xmm.esac.esa.int/xsa/
} 
Tabela 6.1: Propriedades gerais dos aglomerados. Col (1): nome do aglomerado; Col (2): Ascensão reta; Col (3): Declinação; Col (4): Desvio para o vermelho; Col (5): Raio do aglomerado.

\begin{tabular}{ccccc}
\hline \hline Aglomerado & $\begin{array}{c}\alpha \\
(\mathrm{J} 2000)\end{array}$ & $\begin{array}{c}\delta \\
(\mathrm{J} 2000)\end{array}$ & $z$ & $\begin{array}{c}r_{500} \\
h_{70}^{-1} k p c\end{array}$ \\
\hline $\mathrm{A} 496$ & 043337.1 & -131446 & 0.033 & 1480 \\
$\mathrm{~A} 2050$ & 151621.6 & +000559 & 0.1183 & 2172 \\
$\mathrm{~A} 1689$ & 131134.2 & -012156 & 0.1823 & 1785 \\
$\mathrm{~A} 2667$ & 235147.1 & -260018 & 0.23 & 2153 \\
A2631 & 233739.7 & +001737 & 0.273 & 1976 \\
\hline
\end{tabular}

esta seleção dos aglomerados, todos eles, com exceção de A2631, têm aparentemente isofotas simétricas em raios-X, sugerindo que eles são suficientemente relaxados. Desvios do brilho superficial do perfil de A2631, apesar de presentes, não são grandes o bastante para invalidar a hipótese de simetria esférica.

Na tabela (6.1), nós apresentamos os cinco aglomerados de Abell utilizados no trabalho, especificando $r_{500}$, o raio dentro do qual a densidade média excede a densidade crítica $\rho_{\text {crit }}^{2}$ do universo por um fator de 500. Todas as massas foram computadas dentro de $r_{500}$, este é o maior raio para o qual os dados em raios-X não requerem nenhum modelo de extrapolação (Vikhlinin et al. 2006; Lacey \& Cole 1993).

Em geral, a massa dos aglomerados é medida utilizando-se apenas a hipótese de equilíbrio hidrostático, sem levar em conta a contribuição de pressões não térmicas. Neste caso, o cálculo da massa total, necessita apenas dos perfis de densidade e temperatura do gás.

Satélites com melhor resolução espacial (tais como XMM-Newton e Chandra ${ }^{3}$ ) mostram uma diferença significativa entre os dados de brilho superficial e o modelo

\footnotetext{
${ }^{2} \rho_{\text {crit }} \approx 1.88 h^{2} \times 10^{-29} \mathrm{gcm}^{-3}$

${ }^{3}$ http://chandra.harvard.edu/
} 
$\beta$ (Cavaliere \& Fusco-Femiano 1976, 1978) em raios pequenos para aglomerados cool-core (CC) (Jones \& Forman 1984; Xue \& Wu 2000). Devido a esta diferença observacional o modelo $\beta$ foi utilizado para descrever a distribuição de densidade em aglomerados non-cool core (NCC), enquanto o perfil Sérsic (Pislar et al. 1997; Demarco et al. 2003) foi usado para caracterizar os aglomerados CC. Para A2050 A2631, a densidade do gás $\left(\rho_{g}\right)$ é descrita por

$$
\rho_{g}(r)=\rho_{0}\left(1+\frac{r^{2}}{r_{c}^{2}}\right)^{-3 \beta / 2},
$$

onde $\rho_{0}$ e $r_{c}$ são a densidade do gás central e o raio do core, respectivamente. O parâmetro $\beta$ determina o comportamento da lei de potência para grandes raios.

Para A496, A1689 e A2667 (aglomerados CC) o perfil de densidade do gás foi ajustado pelo modelo de Sérsic dado por

$$
\rho_{g}(r)=\rho_{0}\left(\frac{r}{a}\right)^{-p^{\prime}} \exp \left[-\left(\frac{r}{a}\right)^{\nu}\right],
$$

onde $p^{\prime}=p / 2, p=1-0.6097 \nu+0.05563 \nu^{2}$ e $a=a^{\prime} 2^{1 / \nu}$, onde $a, \nu$ e $p$, são parâmetros ajustados pelas observações (Durret et al. 2005; Laganá et al. 2008).

\subsection{O perfil do Campo Magnético}

Não temos medidas de campos magnéticos para os aglomerado de nossa amostra, de forma a vincular precisamente o perfil do campos magnético, sendo necessário uma estimativa baseada nos dados da literatura para outros aglomerados.

Jaffe (1980) sugeriu que a distribuição do campo magnético intraglomerado deveria depender da densidade térmica do gás e da distribuição das galáxias massivas. 
Como conseqüência teríamos um perfil que diminuiria com o raio do aglomerado. Observações podem impor vínculos sobre o gradiente radial deste campo magnético (Brunetti 2001; Govoni et al. 2001; Feretti et al. 2004b). A intensidade destes campos deveria diminuir com o raio em relação à região central do aglomerado de forma similar ao gás intraglomerado.

Utilizando simulações magnetohidrodinâmicas, uma importante caracterização da distribuição de campos magnéticos em aglomerados foi feita por Dolag et al, (1999); Dolag et al. (2002). Estes autores acharam que os campos magnéticos observados no gás intraglomerado poderiam ser reproduzidos pela evolução de um campo magnético inicial em $z=15$, que foi amplificado pela compressão gravitacional durante o colapso do aglomerado. Outro resultado interessante foi que a intensidade do campo magnético em um dado ponto é proporcional à densidade do gás.

Colafrancesco \& Giordano (2007) estudaram a influência dos campos magnéticos nas propriedades de grupos e aglomerados de galáxias virializados, assumindo que eles escalam com a densidade do gás da forma $B(r) \propto \rho^{\alpha}$. A mesma dependência em lei de potência foi usada por Zhang (2004) para estimar o efeito do campo magnético intraglomerado no espectro de potência do efeito Sunyaev-Zel'dovich. Motivados pelos trabalhos mencionados anteriormente, assumimos um perfil de lei de potência para distribuição radial do campo magnético,

$$
B(r)=B_{0}\left(\frac{\rho_{g}(r)}{\rho_{0}}\right)^{\alpha}
$$

onde $B_{0}$ é o valor do campo magnético na região central e $\alpha$ é um parâmetro de forma. A intensidade da pressão magnética $P_{\mathrm{B}}$ pode ser relacionada com a 
intensidade do campo magnético da forma

$$
P_{\mathrm{B}}(r)=\frac{\langle\mathrm{B}(\mathrm{r})\rangle^{2}}{8 \pi} .
$$

Feretti et al. (1999) estimaram que o campo magnético no meio intraglomerado de A119 deveria estar na faixa de $5-10 \mu G$. Bagchi et al. (1998) acharam $B \approx 1 \mu \mathrm{G}$ para a intensidade dos campos magnéticos na escala de aglomerados.

Clarke, Kronberg, \& Böhringer (2001) estudaram uma amostra de 16 aglomerados em $z<0.1$, achando que o meio intraglomerado é permeado com um campo magnético de intensidades de 4-8 $\mu G$. Taylor et al. (1993) acharam grandes valores para os campos centrais, $B \sim 6-30 \mu \mathrm{G}$. Allen (2001) considerou que os valores centrais dos campos magnéticos podem ser de $B=12 \mu \mathrm{G}$ e Carilli \& Taylor (2002) afirmaram que a intensidade dos campos na região central dos aglomerados podem alcançar intensidades de $10-40 \mu \mathrm{G}$.

Levando em conta estes resultados observacionais, consideramos valores de $B$ em acordo com os valores médios da literatura. De forma a testar os efeitos da pressão magnética na determinação da massa, utilizamos valores entre $5-30 \mu G$. Consideramos uma variação nos valores de $\alpha$ baseados nos resultados de Dolag et al. (2001a). Variamos nosso parâmetro entre $0.5<\alpha<0.9$.

\subsection{Turbulência em Aglomerados de Galáxias}

É amplamente aceito que o meio intraglomerado é turbulento, mergers podem ser um dos mecanismos de maior injeção de energia em aglomerados (veja Sarazin 2002; Brunetti 2003; Lazarian 2006, e referências). Em geral estes modelos 
assumem um cenário com escalas de injeção de energia de 100-500 kpc e velocidades da ordem de $10^{3} \mathrm{~km} / \mathrm{s}$.

Uma vez que a taxa de dissipação da energia turbulenta não pode exceder a luminosidade em raios-X $\left(L_{X}\right)$ do aglomerado no estado estacionário, i.e., $\frac{1}{2} v_{0}^{3} / l_{0} \lesssim$ $L_{X} / M_{\mathrm{g}}$, onde $v_{0}$ e $l_{0}$ são as velocidades e escalas de turbulência respectivamente, e $M_{\mathrm{g}}$ é a massa do gás intraglomerado. Temos um limite superior para as velocidades turbulentas como segue (Subramanian 2006a)

$$
v_{0} \lesssim 180 \frac{\mathrm{km}}{\mathrm{s}}\left(\frac{l_{0}}{200 \mathrm{kpc}}\right)^{\frac{1}{3}}\left(\frac{L_{X}}{10^{45} \mathrm{erg} / \mathrm{s}}\right)^{\frac{1}{3}}\left(\frac{M_{\mathrm{g}}}{10^{14} M_{\odot}}\right)^{\frac{1}{3}}
$$

Norman \& Bryan (1999) acharam que o meio intraglomerado torna-se turbulento durante a formação do aglomerado, com velocidades turbulentas da ordem de $\sim 400 \mathrm{~km} / \mathrm{s}$ dentro de $1 \mathrm{Mpc}$ de distância ao centro do aglomerado. Esta turbulência possui vórtices com tamanhos entre 50 e 500 kpc. Usando um modelo de mergers para os aglomerados, Ricker \& Sarazin (2001) acharam uma turbulência em grande escala com vórtices do tamanho de vários kpc e velocidades entre $\sim 100-400 \mathrm{~km} / \mathrm{s}$.

Através de observações em raios-X, Schuecker et al. (2004) argüiram que a escala de turbulência do aglomerado de Coma é de $\sim 100$ kpc. Eles consideraram uma velocidade turbulenta de $\sim 250 \mathrm{~km} / \mathrm{s}$ nesta escala.

Para quantificar a contribuição da pressão turbulenta devido os movimentos randômicos do gás intraglomerado, podemos escrever a seguinte relação para turbulência isotrópica $P_{\text {turb }}$ :

$$
P_{\text {turb }}=\frac{1}{3} \rho_{\mathrm{g}}\left(\sigma_{r}^{2}+\sigma_{t}^{2}\right)
$$

onde $\sigma_{r}$ e $\sigma_{t}$ são as dispersões de velocidade radiais e tangenciais do gás intraglo- 
merado respectivamente. Utilizamos para os perfis de dispersão de velocidades os resultados de simulações numéricas de Lau et al. (2009).

\subsection{Determinação da Massa Incluindo Efeitos das Pressões não Térmicas}

Para estimar a massa dos aglomerados consideramos as componentes gravitacional, magnética, turbulenta e térmica do gás. Assumimos simetria esférica, e contabilizamos o balanço entre a pressão magnética, turbulenta e térmica contra a gravidade. Podemos escrever:

$$
\frac{d\left(P_{\mathrm{g}}+P_{\mathrm{B}}+P_{\mathrm{turb}}\right)}{d r}=-\rho_{g} \frac{G M_{\mathrm{PNT}}(r)}{r^{2}}
$$

onde $P_{\mathrm{g}}=\rho_{\mathrm{g}} \mathrm{k}_{\mathrm{B}} \mathrm{T} / \mu \mathrm{m}_{\mathrm{p}}$ é a pressão do gás à uma temperatura $T$, a pressão magnética $P_{\mathrm{B}}$ é dada por $\left\langle B^{2}\right\rangle / 8 \pi$ e a pressão turbulenta $P_{\text {turb }}$ é $\frac{1}{3} \rho_{\mathrm{g}}\left(\sigma_{r}^{2}+\sigma_{t}^{2}\right)$, $G$ é a constante gravitacional e $M_{\mathrm{PNT}}$ é a massa total dentro de um raio $r$.

Em nossa análise, consideramos a massa dentro de um raio de $r_{500}$. Considerando os efeitos tanto do campo magnético, quanto da turbulência podemos escrever a seguinte expressão para massa do aglomerado

$$
\begin{aligned}
M_{\mathrm{PNT}}(r)= & -\frac{k_{B} T}{G \mu m_{H}} r\left(\frac{\mathrm{d} \ln \rho_{g}}{\mathrm{~d} \ln r}+\frac{\mathrm{d} \ln T}{\mathrm{~d} \ln r}\right) \\
& -\frac{r^{2}}{8 \pi \rho_{g} G} \frac{\mathrm{d} B(r)^{2}}{d r}-\frac{r^{2}}{2 \rho G} \frac{\mathrm{d}}{\mathrm{d} r}\left(\rho_{g} \sigma_{r}^{2}\right)-\frac{r}{G}\left(2 \sigma_{r}^{2}-\sigma_{t}^{2}\right),
\end{aligned}
$$

onde $\mu$ é o peso molecular médio, $m_{H}$ é a massa do hidrogênio. 


\subsection{Resultados}

Na tabela (6.2), nós apresentamos a diferença de massa estimada considerando a influência das pressões não térmicas. A variação na massa $\delta M_{\mathrm{PNT}}$ é dada por

$$
\delta M_{\mathrm{PNT}}=\frac{M_{\mathrm{PNT}}(r)-M(r)}{M(r)} .
$$

Quando a diferença de massa é estimada para baixos valores do campo magnético central (isto é, $B_{0}=5$ e $10 \mu \mathrm{G}$ ), a influência dos termos não térmicos é menor que 5\%, a influência da pressão não térmica é desprezível nestes casos. Entretanto, quando consideramos valores mais altos para o campo central $B_{0}=30 \mu \mathrm{G}$, associado com um parâmetro de forma $\alpha=0.5$, a variação na estimativa da massa do aglomerado pode chegar a $\sim 40 \%$ e os termos não térmicos tornam-se bastante significativos nestes casos.

Podemos analisar estes resultados olhando o perfil de massa calculado para os mesmos. Na fig. 6.3, nós mostramos os perfis de massa para A1689 (um aglomerado CC) e para A2050 (aglomerado NCC). Nestas figuras não podemos distinguir claramente a diferença entre as curvas para as massas determinadas com e sem os temos não térmicos, com os valores de $B_{0}=5$ e $10 \mu \mathrm{G}$, contudo vemos claramente que o perfil difere para valores de $B_{0}=30 \mu \mathrm{G}$ com $\alpha=0.5$. 

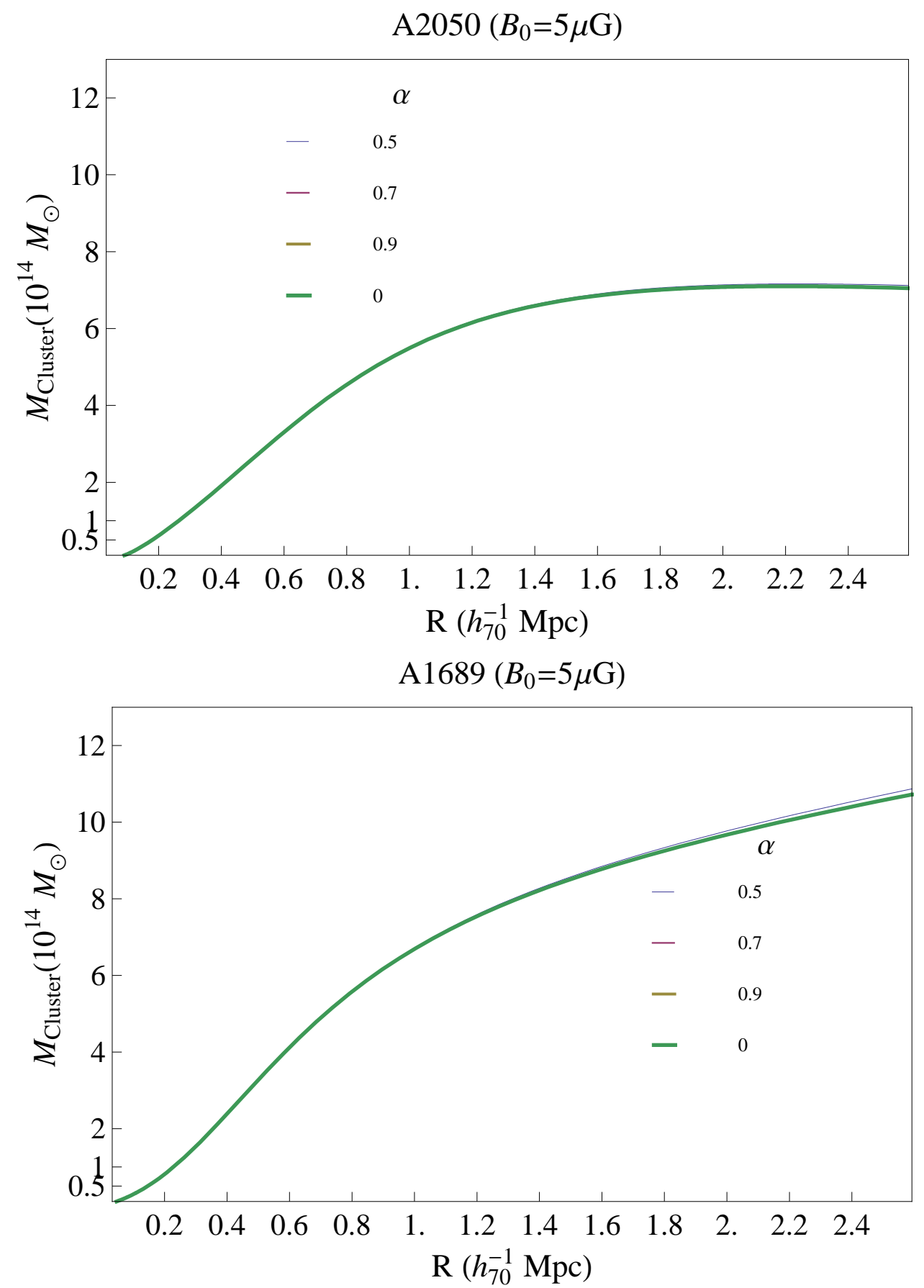

Figura 6.1: Para A2050 e A1689, nós mostramos os perfis de massa determinados sem a consideração da pressão não térmica $P_{\mathrm{NT}}$ (curva verde) comparado com os perfis, incluindo as pressões turbulenta e magnética. Estas três curvas representam os perfis de massa, $\alpha=0.5$ (curva azul), $\alpha=0.7$ (curva rosa) e $\alpha=0.9$ (curva amarela), considerando campos magnéticos centrais de $5 \mu \mathrm{G}$. 
Tabela 6.2: Determinação da massa. Col (1): nome do aglomerado; Col(2): Modelo de perfil de densidade do gás (Laganá et al. 2008); Col.(3), (4) e (5): Determinação da massa incluindo pressão não térmica, assumindo $B_{0}=10 \mu \mathrm{G}$ e $\alpha=0.5,0.7,0.9$, respectivamente. Col.(6), (7) and (8): Determinação da massa, incluindo pressão não térmica assumindo $B_{0}=30 \mu G$ e $\alpha=0.5,0.7,0.9$, respectivamente.

\begin{tabular}{|cc|ccc|ccc|}
\hline Aglomerado & Modelo & \multicolumn{3}{|c|}{$\delta M_{\text {NTP }(10)}(\%)$} & \multicolumn{3}{c|}{$\delta M_{\text {NTP }(30)}(\%)$} \\
\hline & & $\alpha=0.5$ & $\alpha=0.7$ & $\alpha=0.9$ & $\alpha=0.5$ & $\alpha=0.7$ & $\alpha=0.9$ \\
\hline A496 & Sérsic & 0.96 & 0.06 & 0.03 & 34.67 & 2.02 & 0.11 \\
A1689 & Sérsic & 1.12 & 0.07 & 0.004 & 40.14 & 2.49 & 0.14 \\
A2050 & $\beta$ & 0.71 & 0.14 & 0.02 & 25.39 & 5.26 & 1.00 \\
A2631 & $\beta$ & 0.46 & 0.05 & 0.005 & 16.63 & 1.80 & 0.18 \\
A2667 & Sérsic & 0.82 & 0.06 & 0.004 & 29.84 & 2.16 & 0.14 \\
\hline
\end{tabular}



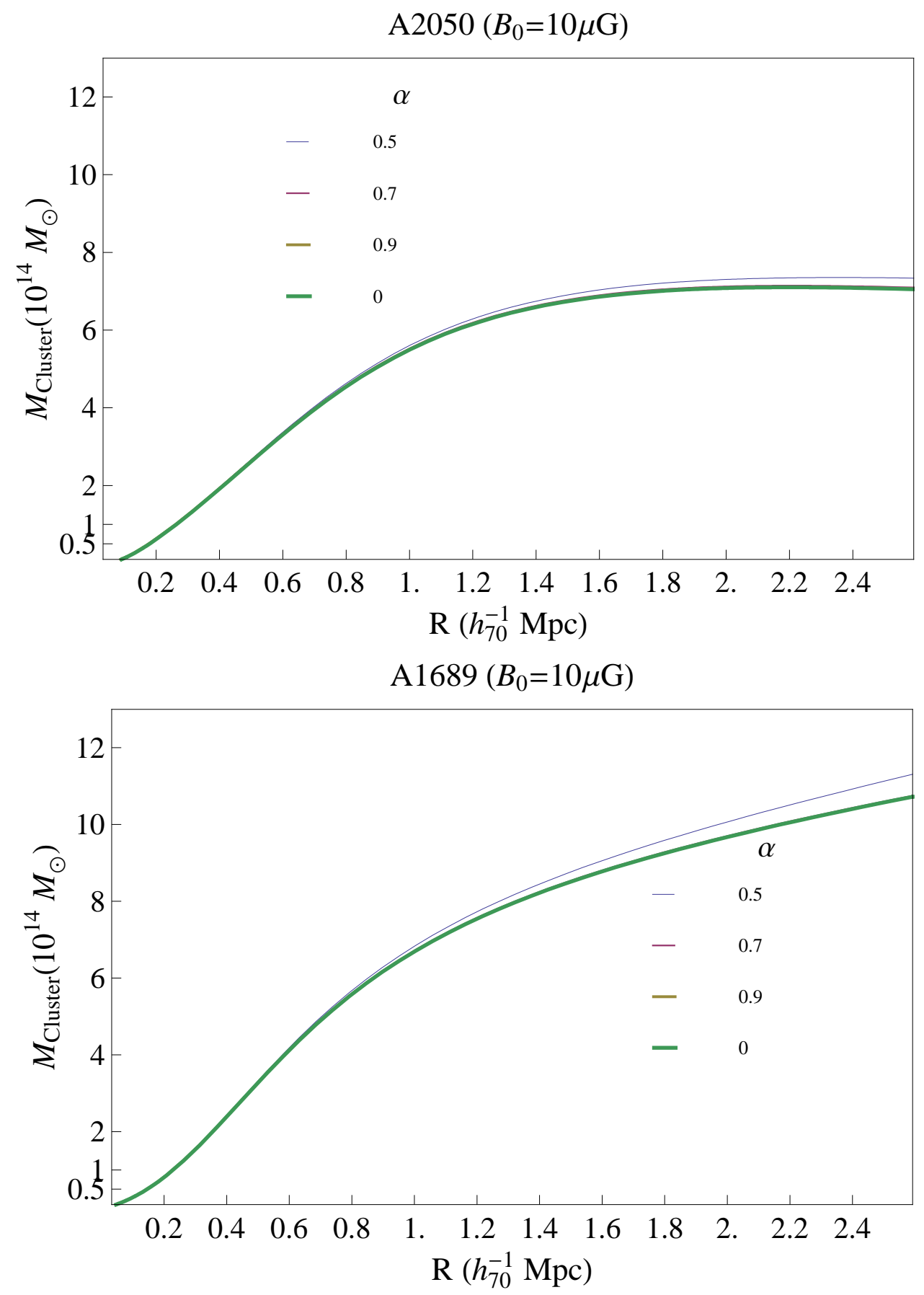

Figura 6.2: Para A2050 e A1689, nós mostramos os perfis de massa determinados sem a consideração da pressão não térmica $P_{\mathrm{NT}}$ (curva verde) comparado com os perfis, incluindo as pressões turbulenta e magnética. Estas três curvas representam os perfis de massa, $\alpha=0.5$ (curva azul), $\alpha=0.7$ (curva rosa) e $\alpha=0.9$ (curva amarela), considerando campos magnéticos centrais de $10 \mu \mathrm{G}$. 

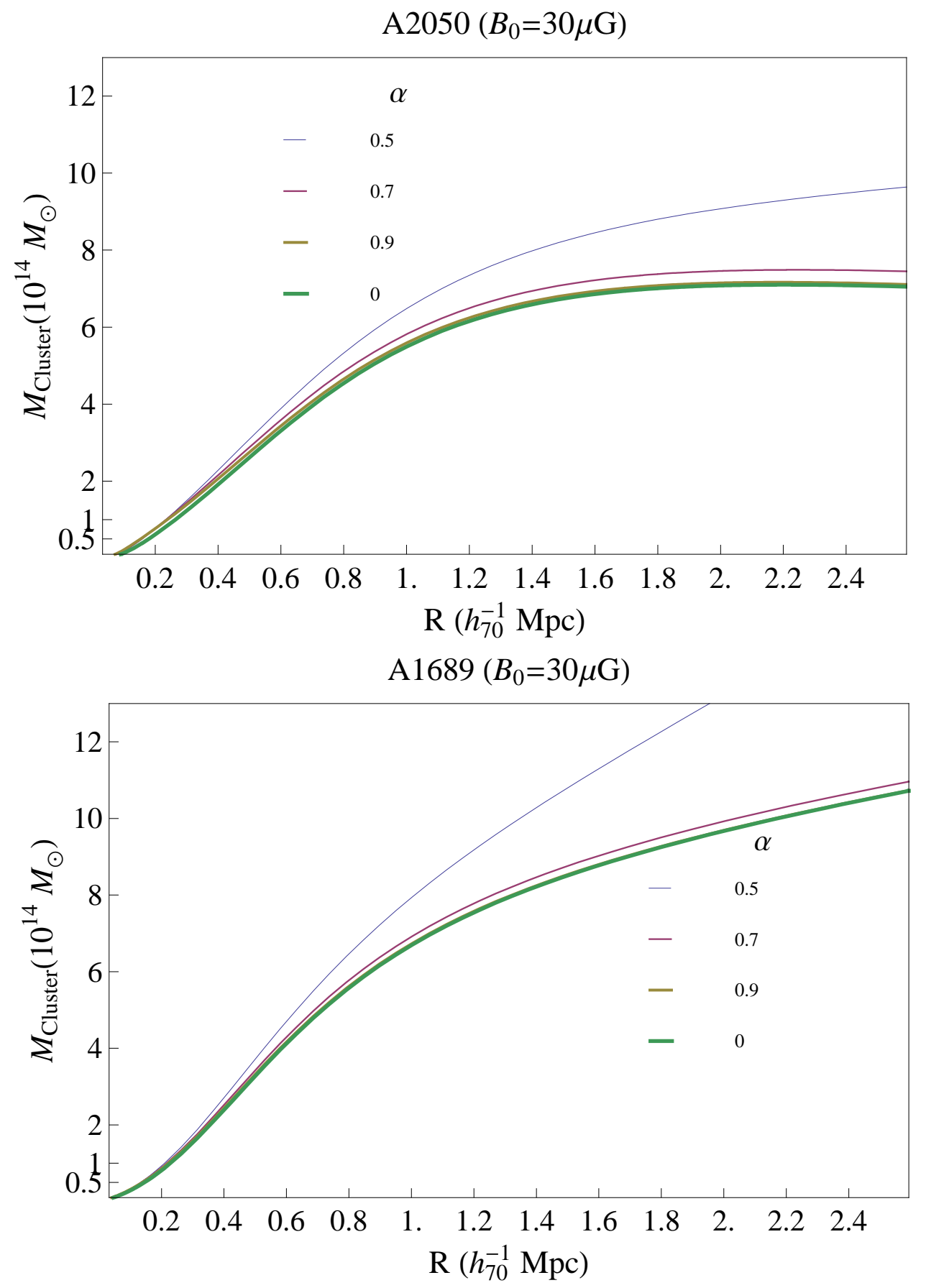

Figura 6.3: Para A2050 e A1689, nós mostramos os perfis de massa determinados sem a consideração da pressão não térmica $P_{\mathrm{NT}}$ (curva verde) comparado com os perfis, incluindo as pressões turbulenta e magnética. Estas três curvas representam os perfis de massa, $\alpha=0.5$ (curva azul), $\alpha=0.7$ (curva rosa) e $\alpha=0.9$ (curva amarela), considerando campos magnéticos centrais de $30 \mu \mathrm{G}$. 
Na fig. 6.4 temos uma melhor visualização da variação da massa em função dos parâmetros centrais e de forma. Podemos verificar que uma mudança considerável na estimativa de massa ocorre apenas para a combinação de baixos valores no parâmetro de forma e altos valores dos campos magnéticos centrais.

Considerando os resultados presentes, vemos que na maioria dos casos a influência destes termos é pequena. Entretanto para alguns casos particulares podemos ter mudanças bastante significativas de até $40 \%$ na determinação da massa. 

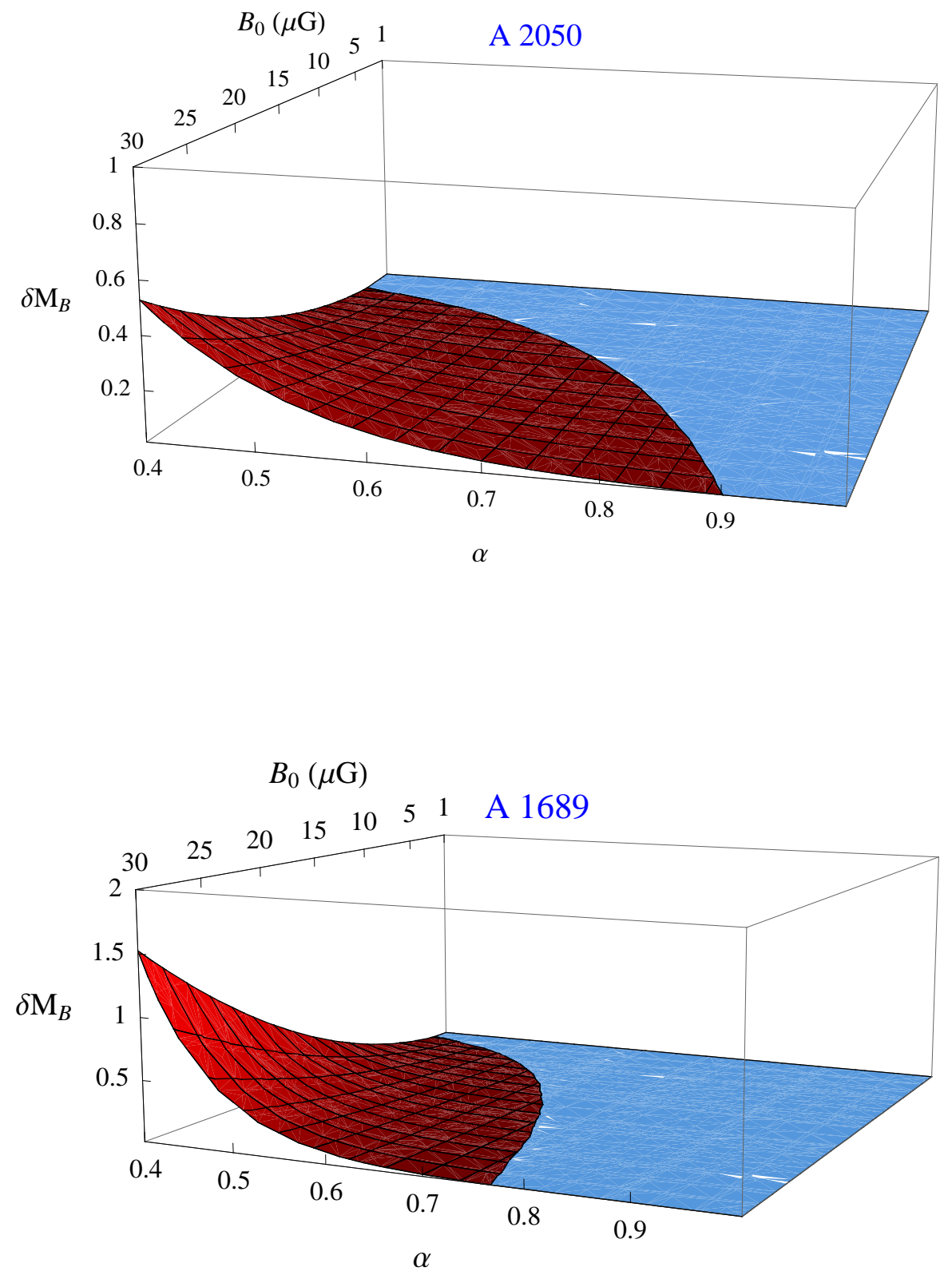

Figura 6.4: Gráficos de A2050, representando os aglomerados NCC e A1689, representando os aglomerados CC. Mostramos o gráfico da variação da massa em função do valor central do campo magnético $B_{0}$, e do parâmetro de forma $\alpha$. 

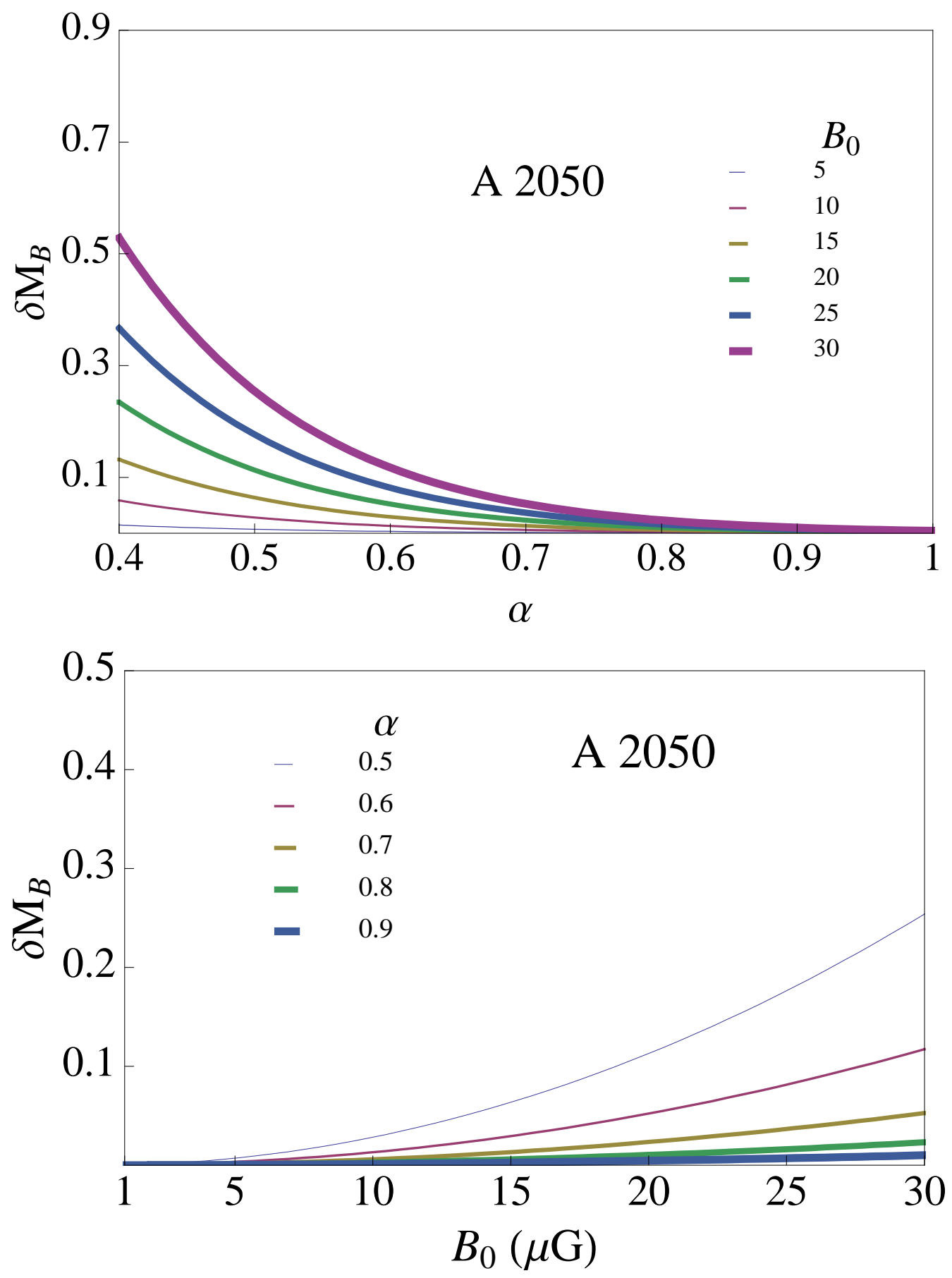

Figura 6.5: Gráficos de A2050, representando os aglomerados NCC. No gráfico superior apresentamos a projeção da variação da massa como função de $\alpha$ para vários valores de $B_{0}$ e no gráfico inferior mostramos a projeção da variação da massa como função do campo magnético central $B_{0}$ para vários valores de $\alpha$. 

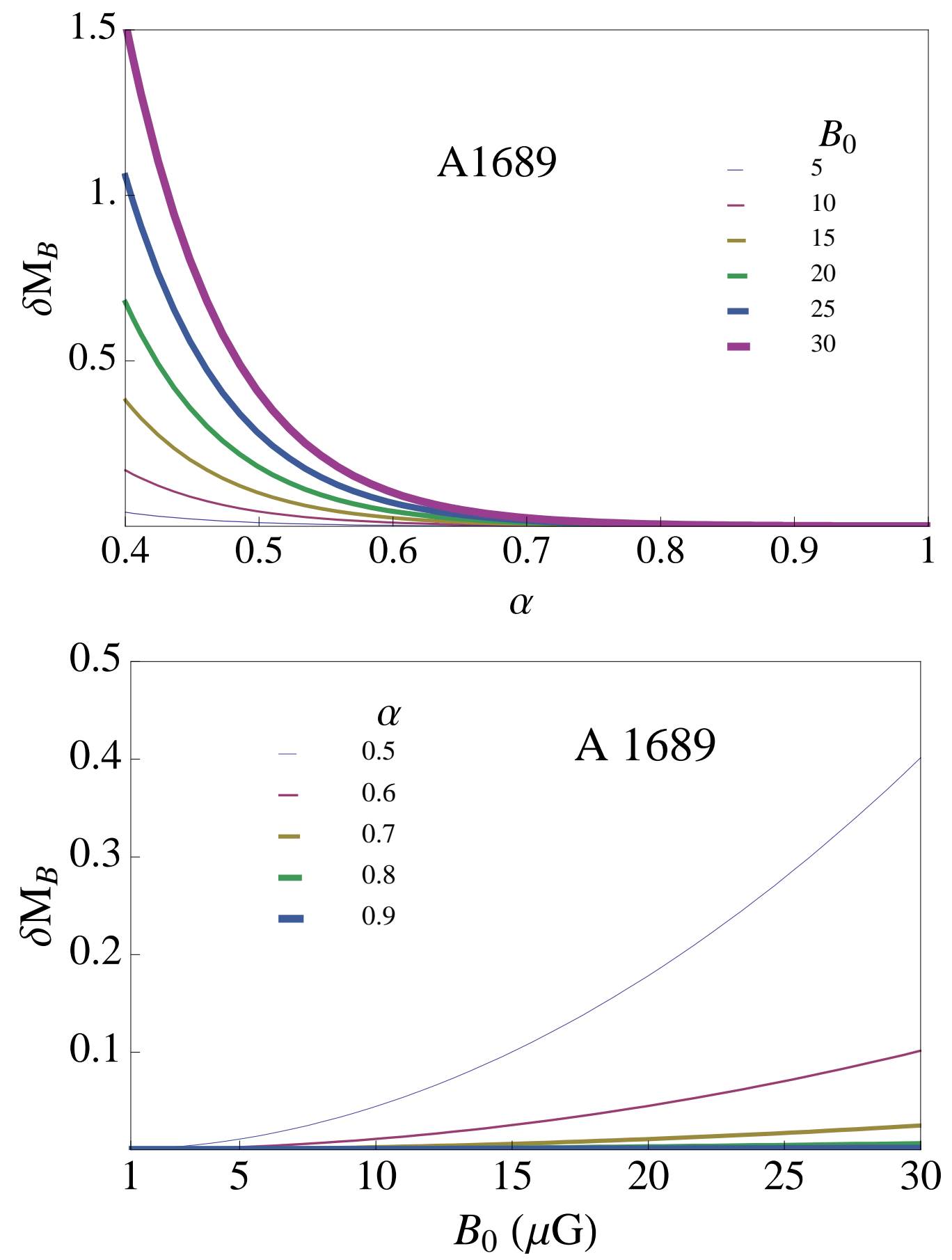

Figura 6.6: Gráficos de A1689, representando os aglomerados CC. No gráfico superior apresentamos a projeção da variação da massa como função de $\alpha$ para vários valores de $B_{0}$ e no gráfico inferior mostramos a projeção da variação da massa como função do campo magnético central $B_{0}$ para vários valores de $\alpha$. 


\subsection{Considerações}

Consideramos os efeitos das componentes magnética e turbulenta na estimativa de massa de 5 aglomerados de Abell. As massas derivadas considerando apenas a pressão térmica foram apresentados no trabalho anterior de Laganá et al. (2008).

Resumimos nossos resultados abaixo:

- A inclusão dos termos de pressão não térmica na descrição do gás intraglomerado é motivada pelas evidências da presença tanto de campos magnéticos quanto de movimentos turbulentos em aglomerados de galáxias. Nós assumimos um perfil para os campos magnéticos dados por $B(r) \propto B_{0} \rho_{g}^{\alpha}$, considerando valores $5 \mu G<B_{0}<30 \mu G$, para cada um destes valores nós variamos o parâmetro de forma, $\alpha=0.5$ até $\alpha=0.9$. Incluímos um termo de turbulência isotrópica $P_{\text {turb }}=\frac{1}{3} \rho_{\mathrm{g}}\left(\sigma_{r}^{2}+\sigma_{t}^{2}\right)$, onde $\rho_{\mathrm{g}}$ é definido nas equações. (6.1) e (6.2) de acordo com o aglomerado. O perfil de velocidades turbulentas foram retirados de simulações numéricas de Lau et al. (2009).

- Considerando $B_{0}=5-10 \mu \mathrm{G}$, a variação da massa, incluindo a pressão não térmica é desprezível. Entretanto, assumindo que o valor central para o campo magnético pode alcançar valores de até $30 \mu \mathrm{G}$, a determinação da massa poderia ser afetada em até $35-40 \%$ se associada ao parâmetro de forma $\alpha=0.5$.

- Este foi o primeiro estudo a considerar os efeitos da pressão não térmica em estimativas de massa de aglomerados reais. É necessária uma maior investigação para uma descrição detalhada das propriedades do gás intraglomerado como apontado por Colafrancesco \& Giordano (2007), a combinação de da- 
dos em raios-X e rádio podem ter um papel importante nesta investigação. A falta de acurácia na determinação da massa de aglomerados pode introduzir um bias nos vínculos cosmológicos de $\Omega_{M}$ baseados em medidas de raios-X destes algomerados. Isto ocorre pois este cálculo é baseado na fração de massa de gás em relação a massa total do aglomerado.

Em uma época onde a quantidade de dados aumenta consideravelmente melhorando cada vez mais nossa amostragem, é imperativo a necessidade de uma melhor análise física dos sistemas estudados, de forma a minimizar um possível viés inserido por uma má modelagem. Deste modo, apesar dos efeitos não térmicos aparentemente não alterarem muito as medidas da massa dos aglomerados, é necessário uma análise mais detalhada de forma a termos uma estimativa acurada da sua real contribuição no suporte de pressão dos mesmos. 


\section{Capítulo 7}

\section{Conclusões e Perspectivas}

Ao longo desse trabalho, analisamos a origem, evolução e importância dos campos magnéticos em diversos ambientes astrofísicos. Mostramos que flutuações eletromagnéticas geradas no universo primordial logo após a TFQH, constitui um forte candidato para origem dos campos magnéticos cosmológicos. Estudamos a evolução destas flutuações iniciais desde $t=10^{-4} \mathrm{~s}$ até $z \sim 10$. Achamos campos de $\sim 10 \mu \mathrm{G}$ sobre um tamanho comóvel de $\sim 1$ pc nesta época. Utilizando nossa previsão primordial, consideramos a possibilidade destes campos servirem de semente para o mecanismo de dínamo em pequena escala existente nas protogaláxias. Achamos campos magnético da ordem de $10^{-9}-10^{-8} \mathrm{G}$ em regiões de 50 kpc comóvel. Estes campos magnéticos durante o processo de formação galáctica, são amplificados de forma concomitante ao colapso da região protogaláctica até as escalas de $\mu \mathrm{G}$ observadas hoje.

É importante notar que nosso modelo sugerido para origem dos campos magnéticos é qualitativamente diferente dos modelos discutidos na seção 2.3. Estas sugestões em geral requerem condições físicas muito especiais. Em nosso modelo, 
entretanto, os campos magnéticos surgem naturalmente de flutuações do plasma primordial, descritos pelo teorema da Flutuação-Dissipação.

Outro tema de nosso interesse, foi a origem de campos magnéticos em objetos compactos. Observações indicam a presença de intensos campos magnéticos em magnetares e possivelmente em GRBs. Teorias astrofísicas usuais tem grande dificuldade em explicar tais campos, deste modo avaliamos a previsão oriunda da conjectura S-B de forma a testar uma explicação alternativa para origem do campo magnético nestes objetos.

Em GRBs, a existência de campos magnéticos de $\sim 10^{15} \mathrm{G}$, poderia explicar o fluxo de Poynting requerido para suprir a energia necessária para explicar os surto de raios- $\gamma$ observados. Contudo não há um bom modelo para explicar a existência destes campos, se consideramos a conjectura S-B verdadeira, podemos explicar tais campos na região do horizonte do buraco negro.

Da mesma forma avaliamos os valores do parâmetro $\beta$ da conjectura S-B em estrelas de nêutrons com altos campos magnéticos denominadas magnetares. Fizemos uma análise estatística de forma a mostrar que a previsão da conjectura S-B não pode ser descartada pelos dados atuais, contudo para uma análise mais robusta faz-se necessário uma maior amostragem de forma a termos estatística suficiente para discriminar diferentes modelos.

Além da origem dos campos magnéticos, estudamos sua importância no suporte gravitacional de aglomerados de galáxias junto com efeitos de turbulência. Vimos como a medida da massa destes aglomerados poderia mudar considerando uma análise mais realista de sua estrutura. A inclusão dos termos de pressão não térmica na descrição do gás intraglomerado é motivada pelas evidências da presença de campos magnéticos e turbulência nestes aglomerados. 
Vimos ao longo desta tese a importância dos campos magnéticos para astrofísica. Campos magnéticos podem influenciar na formação das primeiras estrelas, sendo importantes na época de reionização do universo. Estes campos podem alterar a taxa de formação estelar, assim como sua função de massa inicial, podem modificar os modelos de colapso esférico incluindo direções preferenciais no colapso dependendo de sua geometria, podem deixar assinaturas na radiação cósmica de fundo, assim como influenciar na nucleossíntese primordial. Um dos nossos objetivos futuros é investigar a influência destes campos magnéticos primordiais na formação das primeiras estruturas, assim como possíveis assinaturas destes campos primordiais na radiação cósmica de fundo.

Outro aspecto de nosso interesse, será estudar possíveis maneiras de diferenciar modelos de geração destes campos. Em princípio estes modelos podem ser vinculados por mapas de rotação Faraday feitas pela próxima geração de radio telescópios, tais como o $\mathrm{SKA}^{1}$ e por medidas provenientes da radiação cósmica de fundo pelo satélite Planck².

\footnotetext{
${ }^{1}$ http://www.skatelescope.org/

${ }^{2}$ http://www.rssd.esa.int/index.php?project=Planck
} 


\section{Apêndice A}

\section{Dedução do Teorema da}

\section{Flutuação-Dissipação}

Devemos agora demonstrar que a representação espectral das funções de correlação é determinada pelas propriedades dissipativas do meio. Para isto precisamos avaliar a média do produto $j_{i}^{\dagger}(\mathbf{k}, \omega)$ e $j_{j}(\mathbf{k}, \omega)$. Se o sistema está bem definido em um estado estacionário n, a média quântica é definida como o elemento da matriz diagonal do operador

$$
\left[j_{i}^{\dagger}(\mathbf{k}, \omega) j_{j}\left(\mathbf{k}^{\prime}, \omega\right)\right]_{n m}=\sum_{m} j_{i}^{\dagger}(\mathbf{k}, \omega)_{n m} j_{j}\left(\mathbf{k}^{\prime}, \omega\right)_{n m}
$$

onde o somatório é feito sobre todos os estados quânticos do sistema. Os elementos da matriz do operador $\mathbf{j}_{\mathbf{k}, \omega}$, entre os estados estacionário de energia $E_{m}$ e $E_{n}$ têm a seguinte estrutura

$$
\left(j_{\mathbf{k} \omega}\right)_{n m}=2 \pi\left(j_{\mathbf{k}}\right)_{n m} \delta\left(\omega+\omega_{n m}\right),
$$


onde $\omega_{n m}=\left(E_{n}-E_{m}\right)$ é a freqüência de transição entre os estados $n$ e $m$. Substituindo esta expressão e uma similar para $\left(j_{\mathbf{k} \omega}^{\dagger}\right)_{n m}$ em (A.1), e efetuando uma média obtemos

$$
\begin{array}{r}
\left\langle j_{i}^{\dagger}(\mathbf{k}, \omega) j_{j}\left(\mathbf{k}^{\prime}, \omega^{\prime}\right)\right\rangle=2 \pi\left\langle j_{i}^{\dagger}(\mathbf{k}) j_{j}\left(\mathbf{k}^{\prime}\right)\right\rangle_{\omega} \delta\left(\omega-\omega^{\prime}\right), \\
\left\langle j_{i}^{\dagger}(\mathbf{k}) j_{j}\left(\mathbf{k}^{\prime}\right)\right\rangle_{\omega}=2 \pi \sum_{m n} f\left(E_{n}\right)\left[j_{i}^{\dagger}(\mathbf{k})\right]_{n m}\left[j_{j}\left(\mathbf{k}^{\prime}\right)\right]_{m n} \delta\left(\omega-\omega_{n m}\right),
\end{array}
$$

onde $f\left(E_{n}\right)$ é a função de distribuição estatística para todos os diferentes estados quânticos do sistema. Se consideramos o sistema em equilíbrio, esta função será a distribuição de Gibbs,

$$
f\left(E_{n}\right)=e^{\left(F-E_{n}\right) / k_{B} T} .
$$

Sendo $F$ a energia livre do sistema e $T$ sua temperatura. Devemos conectar a função de correlação (A.3), com a energia absorvida pelo sistema devido a dissipação. Para isto devemos assumir a existência de uma perturbação periódica, com energia $V$ proporcional a $j$, atuando no sistema. Se consideramos $j$ a densidade de corrente elétrica, $V$ assume a forma

$$
V=-\int(\mathbf{A}(\mathbf{r}, t) \cdot \mathbf{j}(\mathbf{r}, t)) d^{3} \mathbf{r}
$$

onde A é o potencial vetor da perturbação. Tomando a transformada espacial de Fourier de $\mathbf{A}$ e $j$, podemos escrever

$$
V=-\frac{1}{2} R e \sum_{k}\left(A_{k}(t) \cdot j_{k}^{\dagger}(t)\right)
$$


onde $A_{k}$ por definição é uma função harmônica do tempo

$$
A_{k}(t)=A_{\mathbf{k} \omega} e^{-i \omega t}
$$

Transições entre diferentes estados do sistema são possíveis devido à ação da perturbação de $V$. Usando as equações (A.2) e (A.7), podemos avaliar os elementos da matriz de perturbação correspondente à transição $n \rightarrow m$ :

$$
V_{n m}=-\pi \sum_{k}\left\{\left(A_{\mathbf{k} \omega} \cdot\left(j_{\mathbf{k}}^{\dagger}\right)_{n m}\right) \delta\left(\omega-\omega_{n m}\right)+\left(A_{\mathbf{k} \omega}^{*} \cdot\left(j_{\mathbf{k}}^{\dagger}\right)_{n m}\right) \delta\left(\omega+\omega_{n m}\right)\right\} .
$$

Como conseqüência, a probabilidade de transição de um sistema por unidade de tempo é igual a

$$
\begin{aligned}
w_{n m} & =\frac{\pi}{2 \hbar^{2}} \sum_{k, k^{\prime}, i, j} A_{i}(k, \omega) A_{j}^{*}\left(k^{\prime}, \omega\right)\left\{j_{i}^{\dagger}(k)_{n m} j_{j}\left(k^{\prime}\right)_{m n} \delta\left(\omega-\omega_{n m}\right)\right. \\
& \left.+j_{i}^{\dagger}(k)_{m n} j_{j}\left(k^{\prime}\right)_{n m} \delta\left(\omega+\omega_{n m}\right)\right\} .
\end{aligned}
$$

Em cada transição de $n \rightarrow m$ o sistema absorve uma energia $\hbar \omega_{m n}$; a fonte desta energia é a perturbação externa. A energia absorvida pelo sistema por unidade de tempo pode ser escrita como

$$
Q_{n}=\sum_{m} w_{n m} \hbar \omega_{n m}
$$

Podemos encontrar a energia média absorvida pelo sistema realizando a média de 
(A.11) sobre todos os estados n:

$$
Q=\sum_{m, n} f\left(E_{n}\right) w_{n m} \hbar \omega_{n m}
$$

Substituindo a equação (A.10) em (A.12) e usando o fato que em sistemas em equilíbrio $f\left(E_{n}\right)$ é dado pela função de Gibbs, podemos escrever

$$
\begin{aligned}
Q & =\frac{\pi \omega}{2 \hbar}\left[e^{\hbar \omega / k_{B} T}-1\right] \sum_{k, k^{\prime}, i, j} A_{i}(k, \omega) A_{j}^{*}\left(k^{\prime}, \omega\right) \\
& \times \sum_{m, n} e^{\left(F-E_{n}\right) / k_{B} T} j_{i}^{\dagger}(k)_{n m} j_{j}\left(k^{\prime}\right)_{m n} \delta\left(\omega-\omega_{n m}\right) .
\end{aligned}
$$

Comparando a expressão anterior com a equação (A.4), nós achamos a seguinte relação entre a energia média absorvida pelo sistema por unidade de tempo e a função de correlação,

$$
Q=\frac{\omega}{4 \hbar}\left[e^{\hbar \omega / k_{B} T}-1\right] \sum_{k, k^{\prime}, i, j} A_{i}(k, \omega) A_{j}\left(k^{\prime}, \omega\right)\left\langle j_{i}^{\dagger}(k) j_{j}\left(k^{\prime}\right)\right\rangle_{\omega} .
$$

Por outro lado, a energia absorvida $Q$ pode ser conectada com parâmetros macroscópicos, característicos das propriedades dissipativas do sistema. Quando não existe perturbação externa, temos $\langle j\rangle=0$. A ação da perturbação (A.6), gera uma média não nula de $j$, o qual está relacionada com a magnitude do potencial perturbativo $A$,

$$
j_{i}=\sum_{i} \hat{\alpha}_{i j} A_{j}
$$

onde $\hat{\alpha}_{i j}$ é um operador linear espaço-temporal. Podemos escrever a relação em 
termos das suas componentes de Fourier

$$
j_{i}(\mathbf{k}, \omega)=\sum_{j} \alpha_{i j}(\mathbf{k}, \omega) A_{j}(\mathbf{k}, \omega)
$$

onde $\alpha_{i j}(\mathbf{k}, \omega)$ é um tensor caracterizando as propriedades dissipativas do meio, denominado tensor resposta do sistema. A mudança de energia interna de será igual a média da derivada temporal do hamiltoniano do sistema. Como no hamiltoniano, apenas a perturbação $V$ depende explicitamente do tempo, a mudança da energia interna do sistema pode ser escrita como

$$
\frac{\partial U}{\partial t}=-\int(\dot{A}(r, t) \cdot j(r, t)) d^{3} r
$$

Para obter a energia média por unidade de tempo da energia absorvida $Q$, podemos usar a Eq. (A.15), e efetuar uma média da expressão anterior. Podemos escrever $Q$ da forma

$$
Q=\frac{1}{4} i \omega \sum_{k, i, j}\left(\alpha_{i j}^{*}-\alpha_{j i}\right) A_{i}(\mathbf{k}, \omega) A_{j}^{*}(\mathbf{k}, \omega) .
$$

Comparando esta equação com a (A.14), nós achamos que

$$
\left\langle j_{i}^{\dagger}(k) j_{j}\left(k^{\prime}\right)\right\rangle_{\omega}=\frac{8 \pi^{3} \hbar}{e^{\hbar \omega / k_{B} T}-1} i\left(\alpha_{i j}^{*}-\alpha_{i j}\right) \delta\left(k-k^{\prime}\right) .
$$

Esta expressão nos dá uma conexão entre a função de correlação das flutuações das quantidades físicas do nosso sistema e de suas propriedades características, representadas pelos coeficientes de $\alpha_{i j}$. Usando as equação (A.3) e (3.5), podemos 
escrever a seguinte expressão para a densidade espectral das flutuações de corrente

$$
\left\langle j_{i} j_{j}\right\rangle_{\mathbf{k} \omega}=\frac{\hbar}{e^{\hbar \omega / k_{B} T}-1} i\left[\alpha_{i j}^{*}(\omega, \mathbf{k})-\alpha_{i j}(\omega, \mathbf{k})\right] .
$$

Esta expressão é denominada relação de flutuação dissipação, e determina completamente as flutuações de um sistema em equilíbrio. 


\section{Apêndice B}

\section{Evolução do Tensor de Correlação das Flutuações Magnéticas}

Mostraremos alguns passos principais da derivação destas equações, de forma a dar uma idéia geral da análise feita por Subramanian (1999). Sabemos que

$$
\begin{aligned}
\left(\partial M_{i j} / \partial t\right) & =(\partial / \partial t)\left(<\delta B_{i}(\mathbf{x}, t) \delta B_{j}(\mathbf{y}, t)>\right) \\
& =\left[(\partial / \partial t)\left(<B_{i} B_{j}>\right)-(\partial / \partial t)\left(<B_{i}><B_{j}>\right)\right]
\end{aligned}
$$

O segundo termo pode ser avaliado usando a equação do campo médio. O primeiro termo pode ser calculado usando a equação (4.15) e o fato de que

$$
(\partial / \partial t)\left(B_{i}(\mathbf{x}, t) B_{j}(\mathbf{y}, t)\right)=B_{i}(\mathbf{x}, t)\left(\partial B_{j}(\mathbf{y}, t) / \partial t\right)+\left(\partial B_{i}(\mathbf{x}, t) / \partial t\right) B_{j}(\mathbf{y}, t)
$$

A equação resultante pode ser resolvida iterativamente pegando a equação para $\left(\partial M_{i j} / \partial t\right)$ o qual depende das correlações do campo de velocidades turbulento $T_{i j}$, 
do campo de velocidades médio $\mathbf{v}_{0}$ e do campo magnético médio $\mathbf{B}_{0}$ e dos termos não lineares oriundos dos efeitos da difusão ambipolar. Temos então

$$
\begin{aligned}
\frac{\partial M_{i j}}{\partial t} & =<\int{ }^{y} R_{j p q}\left[v_{T}^{p}(\mathbf{y}, t){ }^{x} R_{i l m}\left(v_{T}^{l}(\mathbf{x}, s)\left[M_{m q}+B_{0}^{m}(\mathbf{x}) B_{0}^{q}(\mathbf{y})\right)\right] d s>\right. \\
& +<\int{ }^{x} R_{i p q}\left[v_{T}^{p}(\mathbf{x}, t){ }^{y} R_{j l m}\left(v_{T}^{l}(\mathbf{y}, s)\left[M_{q m}+B_{0}^{q}(\mathbf{x}) B_{0}^{m}(\mathbf{y})\right)\right] d s>\right. \\
& +<\int{ }^{y} R_{j p q}\left(v_{T}^{p}(\mathbf{y}, t){ }^{y} R_{q l m}\left(v_{T}^{l}(\mathbf{y}, s) M_{i m}\right)\right) d s> \\
& +<\int{ }^{x} R_{i p q}\left(v_{T}^{p}(\mathbf{x}, t){ }^{x} R_{q l m}\left(v_{T}^{l}(\mathbf{x}, s) M_{m j}\right)\right) d s> \\
& +\eta\left[\nabla_{y}^{2} M_{i j}+\nabla_{x}^{2} M_{i j}\right]+{ }^{y} R_{j p q}\left(v_{0}^{p}(\mathbf{y}) M_{i q}\right)+{ }^{x} R_{i p q}\left(v_{0}^{p}(\mathbf{x}) M_{q j}\right) \\
& +{ }^{y} R_{j p q}\left(<v_{D}^{p}(\mathbf{y}) \delta B_{i}(\mathbf{x}) B_{q}(\mathbf{y})>\right)+{ }^{x} R_{i p q}\left(<v_{D}^{p}(\mathbf{x}) B_{q}(\mathbf{x}) \delta B_{j}(\mathbf{y})>\right),
\end{aligned}
$$

onde definimos os operadores

$$
{ }^{x} R_{i p q}=\epsilon_{i l m} \epsilon_{m p q}\left(\partial / \partial x^{l}\right) \text { e }{ }^{y} R_{i p q}=\epsilon_{i l m} \epsilon_{m p q}\left(\partial / \partial y^{l}\right) .
$$

O primeiro termo da equação (B.3) é dado por

$$
\begin{gathered}
<\int{ }^{y} R_{j p q}\left(v_{T}^{p}(\mathbf{y}, t){ }^{x} R_{i l m}\left(v_{T}^{l}(\mathbf{x}, s) M_{m q}\right)\right) d s>= \\
-\epsilon_{i t u} \epsilon_{u l m} \epsilon_{j r s} \epsilon_{s p q} \frac{\partial^{2}}{\partial r^{r} \partial r^{t}}\left[T^{l p} M_{m q}\right]
\end{gathered}
$$

Para examinar a evolução de $M_{L}$ devemos multiplicar as equações anteriores por $r^{i} r^{j} / r^{2}$. Podemos simplificar a equação resultante usando a seguinte identidade

$$
r^{i} r^{j} \frac{\partial^{2} A}{\partial r^{r} \partial r^{t}}=\frac{\partial^{2}\left(A r^{i} r^{j}\right)}{\partial r^{r} \partial r^{t}}-\delta_{j t} r^{i} \frac{\partial A}{\partial r^{r}}-\delta_{i r} r^{j} \frac{\partial A}{\partial r^{t}}-\delta_{j t} \delta_{i r} A,
$$


onde $A=T^{l p} M_{m q}$. Então usando $\epsilon_{i t u} \epsilon_{u l m}=\delta_{i l} \delta_{t m}-\delta_{i m} \delta_{t l}$, e a definição de $T_{L L}, T_{N N}$ e $C$, podemos chegar na contribuição do primeiro termo de (B.3)

$$
\frac{\partial M_{L}}{\partial t}=-\frac{1}{r^{4}} \frac{\partial}{\partial r}\left(r^{4} T_{L L} \frac{\partial M_{L}}{\partial r}\right)+\frac{G}{2} M_{L}+4 C H .
$$

O segundo termo da equação (B.3) dá uma contribuição idêntica.

Para derivar a evolução de $H$ devido estes termos, multiplicamos a equação (B.5) por $\epsilon_{i j f} r^{f}$. Usando o fato que a velocidade turbulenta e o campo em pequena escala têm divergência nula, temos então $M_{i j, j}=0$ e $T_{i j, j}=0$. Simplificando a contribuição do primeiro termo de (B.3)

$$
\frac{\partial H}{\partial t}=-\frac{\epsilon_{i j f} r^{f}}{2 r^{2}}\left[T_{i j, t r} M_{t r}+T_{t r} M_{i j, t r}-T_{i r, t} M_{t j, r}-T_{t j, r} M_{i r, t}\right]
$$


Os primeiros dois termos da equação (B.8) podem ser simplificados notando que $\epsilon_{i j f} T_{i j}=2 C r^{f}$ e $\epsilon_{i j f} M_{i j}=2 H r^{f}$. Temos então

$$
\begin{aligned}
& -\frac{\epsilon_{i j f} r^{f}}{2 r^{2}}\left[T_{i j, t r} M_{t r}+T_{t r} M_{i j, t r}\right]= \\
& -\left[T_{L L} H^{\prime \prime}+T_{L L}^{\prime} H^{\prime}+\frac{4 T_{L L} H^{\prime}}{r}+M_{L} C^{\prime \prime}+M_{L}^{\prime} C^{\prime}+\frac{4 M_{L} C^{\prime}}{r}\right],
\end{aligned}
$$

onde o sinal ' denota derivada com respeito a $r$. Para avaliar a contribuição dos últimos dois termos da equação (B.8) é conveniente abrir os tensores $M_{i j}$ e $T_{i j}$ em uma parte simétrica e uma anti-simétrica. Utilizamos o índice $S$ para parte simétrica e $A$ para parte anti-simétrica. Desta forma podemos escrever

$$
\begin{aligned}
& \frac{\epsilon_{i j f} r^{f}}{2 r^{2}}\left[T_{i r, t} M_{t j, r}+T_{t j, r} M_{i r, t}\right]=\frac{\epsilon_{i j f} r^{f}}{r^{2}}\left[T_{i r, t}^{S} M_{t j, r}^{A}+T_{i r, t}^{A} M_{t j, r}^{S}\right] \\
& =-\left[H T_{L L}^{\prime \prime}+C M_{L}^{\prime \prime}+T_{L L}^{\prime} H^{\prime}+M_{L}^{\prime} C^{\prime}+\frac{4 H T_{L L}^{\prime}}{r}+\frac{4 C M_{L}^{\prime}}{r}\right] .
\end{aligned}
$$

Adicionando as contribuições das equação (B.9) e (B.10) temos

$$
\frac{\partial H}{\partial t}=-\frac{1}{r^{4}} \frac{\partial}{\partial r}\left(r^{4} \frac{\partial}{\partial r}\left[T_{L L} H+C M_{L}\right]\right.
$$

O segundo termo da equação (B.3) gera uma contribuição idêntica.

Os primeiros dois termos da equação (B.3) representam o efeito das correlações de velocidade nas flutuações magnéticas $\left(M_{i j}\right)$ e no campo médio $\left(B_{0}^{i}\right)$. Os dois termos seguintes estão ligados ao transporte turbulento das flutuações pelo campo de velocidades turbulento, o quinto e sexto termos representam os efeitos de difusão. Os sétimo e oitavo termos representam o transporte das flutuações magnéticas pelo campo de velocidades médio. Os últimos dois termos são devido 
aos efeitos da difusão ambipolar.

De forma a obter equações para $M_{L}$ e $H$, multiplicamos a equação (B.3) por $\left(r^{i} r^{j}\right) / r^{2}$ e $\epsilon_{i j f} r^{f}$ e usamos a identidade:

$$
M_{L}(r)=M_{i j}\left(r^{i} r^{j} / r^{2}\right), H(r)=M_{i j} \epsilon_{i j f} r^{f} /\left(2 r^{2}\right)
$$

O terceiro e quarto termos adicionam a seguinte contribuição

$$
4 C(0) \epsilon_{j q m}\left(\partial M_{i m} / \partial r^{q}\right)+2 T_{L L}(0) \nabla^{2} M_{i j}
$$

na equação (B.3), justificando assim o termo transporte turbulento de $M_{i j}$. Devemos avaliar os últimos dois termos devido a difusão ambipolar. Eles adicionam uma contribuição de $-8 a H(0, t) \epsilon_{j q m}\left(\partial M_{i m} / \partial r^{q}\right)+4 a M_{L}(0, t) \nabla^{2} M_{i j}$ na Eq. (B.3). Juntando todos os termos, nós chegamos em uma equação acoplada para evolução de $M_{L}$ e $H$ :

$$
\begin{gathered}
\frac{\partial M_{L}}{\partial t}=\frac{2}{r^{4}} \frac{\partial}{\partial r}\left(r^{4} \kappa_{N} \frac{\partial M_{L}}{\partial r}\right)+G M_{L}-4 \alpha_{N}, H \\
\frac{\partial H}{\partial t}=\frac{1}{r^{4}} \frac{\partial}{\partial r}\left(r^{4} \frac{\partial}{\partial r}\left(2 \kappa_{N} H+\alpha_{N} M_{L}\right)\right),
\end{gathered}
$$

onde nós definimos:

$$
\begin{aligned}
& \kappa_{N}=\eta+T_{L L}(0)-T_{L L}(r)+2 a M_{L}(0, t), \\
& \alpha_{N}=2 C(0)-2 C(r)-4 a H(0, t), \\
& G=-4\left[\frac{d}{d r}\left(\frac{T_{N N}}{r}\right)+\frac{1}{r^{2}} \frac{d}{d r}\left(r T_{L L}\right)\right] .
\end{aligned}
$$

Estas equações formam um conjunto de fechado de equações a derivadas parciais para evolução das correlações do campo magnético. 
APÊNDICE B. EVOLUÇÃO DO TENSOR DE CORRELAÇÃO DAS FLUTUAÇÕES MAGNÉTICAS 


\section{Apêndice C}

\section{Modelo Cosmológico Padrão}

\section{C.1 Métrica de Friedman-Robertson-Walker}

Evidências observacionais como a isotropia da radiação cósmica de fundo nos levam a crer que o universo é um hiperespaço quadridimensional composto por uma direção temporal e uma variedade tridimensional espacial homogênea e isotrópica.

A métrica de Friedman-Robertson-Walker (FRW) pode ser obtida partindo apenas dessas hipóteses.

O elemento de linha mais geral para um universo homogêneo e isotrópico é dado por:

$$
d s^{2}=d t^{2}-a^{2}(t)\left[\frac{1}{1-k r^{2}} d r^{2}+r^{2}\left(d \theta^{2}+\operatorname{sen}^{2} \theta d \phi^{2}\right)\right],
$$

onde $a(t)$ é o fator de escala da expansão e $t$ o tempo no referencial de um observador comóvel (cujo deslocamento se deve apenas à expansão), também chamado 
tempo cósmico.

A partir da equação (C.1), e de posse do formalismo da Relatividade Geral, onde o elemento de linha é dado por:

$$
d s^{2}=g_{\mu \nu} d x^{\mu} d x^{\nu}
$$

podemos reconhecer a métrica FRW no tensor métrico representado por:

$$
g_{\mu \nu}=\left(\begin{array}{cccc}
1 & 0 & 0 & 0 \\
0 & -\frac{a^{2}(t)}{1-k r^{2}} & 0 & 0 \\
0 & 0 & -a^{2}(t) r^{2} & 0 \\
0 & 0 & 0 & -a^{2}(t) r^{2} \operatorname{sen}^{2} \theta
\end{array}\right) .
$$

\section{C.2 Tensor Energia-Momento}

O conteúdo do universo pode ser aproximado por um fluido perfeito, onde existe interação entre as partículas mas não há troca de calor nem viscosidade. A forma mais simples do tensor energia-momento para um fluido com essas características pode ser escrita como:

$$
T^{\mu \nu}=\rho u^{\mu} u^{\nu}+p S^{\mu \nu}, \quad \text { onde } \quad S^{\mu \nu}=a u^{\mu} u^{\nu}+b g^{\mu \nu} .
$$

Aqui, $a$ e $b$ são constantes. Impondo que as leis de conservação de energia e momento $\left(\nabla_{\beta} T^{\alpha \beta}=0\right)$ sejam respeitadas, o tensor energia-momento para um fluido perfeito é dado por: 


$$
T^{\mu \nu}=(\rho+p) u^{\mu} u^{\nu}-p g^{\mu \nu},
$$

ou seja,

$$
T^{\mu}{ }_{\nu}=\left(\begin{array}{cccc}
\rho & 0 & 0 & 0 \\
0 & -p & 0 & 0 \\
0 & 0 & -p & 0 \\
0 & 0 & 0 & -p
\end{array}\right) .
$$

A componente temporal das equaçõess de conservação resulta em:

$$
\dot{\rho}+3 \frac{\dot{a}}{a}(\rho+p)=0,
$$

onde o ponto representa a derivada em relação ao tempo cósmico.

A homogeneidade espacial nos garante que tanto a pressão como a densidade são funções apenas do tempo.

\section{C.3 Equações de Einstein}

Com o intuito de estudar a dinâmica envolvida na evolução temporal de um universo homogêneo e isotrópico, devemos utilizar a métrica de FRW e o tensor energia-momento junto com as equações de Einstein, dadas por:

$$
R_{\mu \nu}-\frac{1}{2}(R+2 \Lambda) g_{\mu \nu}=8 \pi G T_{\mu \nu},
$$


onde $R_{\mu \nu}$ é o tensor de Ricci, $R$ o escalar de curvatura e $\Lambda$ a constante cosmológica.

As componentes do tensor de Ricci são dadas por:

$$
\begin{aligned}
& R_{00}=3 \frac{\ddot{a}}{a}=-4 \pi G(\rho+p)+\Lambda, \\
& R_{0 i}=0 \\
& R_{i j}=-\frac{g_{i j}}{a^{2}}\left[2 k+\left(a \ddot{a}+2 \dot{a}^{2}\right)\right]=4 \pi G g_{i j}(p-\rho)+g_{i j} \Lambda .
\end{aligned}
$$

Utilizando estas expressões junto com (C.4) na equação (C.6), temos:

$$
\begin{gathered}
H^{2}=\left(\frac{\dot{a}}{a}\right)^{2}=\frac{8 \pi G}{3} \rho-\frac{k}{a^{2}}+\frac{\Lambda}{3} \\
\frac{\ddot{a}}{a}=-\frac{4 \pi G}{3}(\rho+3 p)+\frac{\Lambda}{3} .
\end{gathered}
$$

A expressão (C.10) é chamada equação de Friedman, $H$ é conhecido como parâmetro de Hubble e a equação (C.11) como equação da aceleração. Nessas expressões, a densidade e a pressão correspondem a todo o conteúdo material do universo. Caso este possua mais de uma componente, $\rho$ e $p$ devem ser substituídos por suas respectivas somatórias sobre os constituintes em questão.

As equações de Einstein descrevem a evolução do universo com base em duas equações (equações (C.10) e (C.11)) e três incógnitas ( $a, \rho$ e $p$ ). Logo, precisamos de uma terceira equação independente das duas anteriores para encontrar a solução 
do sistema. Para isto, podemos utilizar uma equação de estado

$$
p=w \rho,
$$

onde $w$ varia conforme o constituinte considerado.

Podemos definir a densidade crítica de energia, que pode ser utilizada para comparar as contribuições de cada componente para a densidade de energia total do universo. Podemos obtê-la dividindo a expressão (C.10) por $H^{2}$ :

$$
1=\frac{8 \pi G}{3 H^{2}} \rho_{m}-\frac{k}{a^{2} H^{2}}+\frac{\Lambda}{3 H^{2}}
$$

A densidade crítica é definida como a densidade total de energia em um universo com curvatura nula e sem constante cosmológica, logo,

$$
\rho_{c r}=\frac{3 H^{2}}{8 \pi G}
$$

A partir dela, podemos definir um parâmetro de densidade associado com cada constituinte, matéria, constante cosmológica, curvatura e radiação

$$
\Omega_{m}=\frac{\rho_{m}}{\rho_{c r}}, \quad \Omega_{\Lambda}=\frac{\Lambda}{3 H^{2}}, \quad \Omega_{k}=-\frac{k}{a^{2} H^{2}}, \quad \Omega_{r}=\frac{32 \pi G \sigma T}{3 H^{2} c^{3}}
$$

assim, podemos escrever:

$$
1-\Omega_{k}=\Omega_{\text {total }}=\Omega_{m}+\Omega_{\Lambda}
$$


Dados de cinco anos do WMAP, combinados com observações de Oscilações Acústicas de Bárions (Baryon Acoustic Oscillations-BAO) e supernovas do Tipo Ia, indicam valores de $H_{0}=70.5 \pm 1.3 \mathrm{~km} / \mathrm{s} / \mathrm{Mpc}, \Omega_{m} h^{2}=0.1358_{-0.0036}^{+0.0037},-0.0179<$ $\Omega_{k}<0.0081$ e $\Omega_{\Lambda}=0.726 \pm 0.015$, onde $H=100 \mathrm{kms}^{-1} / \mathrm{Mpc}$ (Komatsu et al. 2009). 


\section{Referências Bibliográficas}

Abassi, R. V. et al., ApJ, 610, L73 (2004).

Ahonen, J. \& Enqvist, K., Phys. Lett. B, 382, 40, (1996).

Akhiezer, A. I., Plovin, R. V., Sitenko, A. G., \& Stepanov, K. N., Plasma Electrodynamics (Pergamon: Oxford 1975).

Alfvén, H., Ark. Mat. Astron. Fys., 25B, 29, (1937).

Alfvén, H., Z. Physik, 107, 579, (1937).

Allen, S. W., Taylor, G. B., Nulsen, P. E. J., et al., MNRAS, 324, 842, (2001).

Andernach, H., Han Tie, Sievers, A., et al., A\&A, 73, 265, (1988).

Arge, C. N., Mullan, D. J. \& Dolginov, A. Z., ApJ, 443, 795, (1995).

Atteia, J.-L., Boer, M., Hurley, K., et al., ApJ, 320, L105, (1987).

Bagchi, J., Pislar, V., \& Lima Neto, G. B., MNRAS, 296, L23, (1998).

Bagchi, J. Ensslin, T. A., Miniati, F., Stalin, C. S., Singh, M., Raychaudhury, S., Humeshkar, N. B., New Astron., 7, 249, (2002).

Banerjee, R. \& Jedamzik, K., Phys. Rev. D, 70, 123003, (2004).

Barkana, R. \& Loeb, A., Phys. Repts. 349, 125, (2001).

Barnes, I., \& Efstathiou, G., ApJ, 319, 575, (1987).

Barut, A. O. and Gornitz, T., Found. Phys., 15, 433, (1985).

Baym, G., Bödeker, D., \& McLerran, L., Phys. Rev. D, 53, 662, (1996).

Beck, R., Branderburg, A., Moss, D., Shukurov, A., and Sokoloff, D., ARA\&A, 34, 155, 
(1996).

Beck, R., AIP Conference Proceedings, 1085, 83, (2008).

Bennet et al., Proc. R. Soc. London A, 198, 39, (1949).

Biermann, L., Z. Naturforsch., 5a, 65, (1950).

Biskamp, D. \& Welter, H., Phys. Rev. Lett., 44, 16, (1980).

Blackett, P. M. S., Nature, 159, 658, (1947).

Blandford, R. D., \& Znajek, R. L., Mon. Not. R. Astron. Soc. 179, 433, (1977).

Blasi, P., Burles, S., \& Olinto, A. V., ApJ, 514, 79, (1999).

Boyanovsky, D., NATO Advanced Study Institute on Phase Transitions in the Early Universe: Theory and Observations, ed. de Vega, H. J., Khalatnikov, M., \& Snachez, N. (Kluwer: Dordrecht/London 2001).

Boyd, T. J. M. \& Sanderson, J. J., The Physics of Plasmas(Cambridge University Press, 2003).

Braginskii, S. I., Rev. Plas. Phys., 1, 205, (1965).

Brandenburg, A. and Subramanian, K., A\&A, 361, L33, (2000).

Brandenburg, A. and Subramanian, K., Phys. Rep., 417, 1, (2005).

Brunetti, G., Astronomical Society of the Pacific Conference Series, ed. S. Bowyer \& C.-Y. Hwang, 349, (2003).

Brunetti, G., Setti, G., Feretti, L., \& Giovannini, G., MNRAS, 320, 365, (2001).

Buren, V., ApJ, 294, 567, (1985).

Carilli, C. L. \& Taylor, G. B., A\&RA, 40, 319, (2002).

Cavaliere, A. \& Fusco-Femiano, R., A\&A, 49, 137, (1976).

Cavaliere, A. \& Fusco-Femiano, R., A\&A, 70, 677, (1978).

Cen, R., ApJ, 591, 12, (2003).

Cheng, B., \& Olinto, A. V., Phys. Rev. D, 50, 2421, (1994).

Ciardi, B., Ferrara, A., White, S. D. M., MNRAS, 344, L7, (2003). 
Clarke, T. E., Kronberg, P. P., \& Böhringer, H., ApJ, 547, 111, (2001).

Cocconi, G., Nuovo Cimento, 3, 1422, (1956).

Colafrancesco, S. \& Giordano, F., A\&A, 466, 421, (2007).

Cooke, J., et al., The Astronomy and Astrophysics Decadal Survey, Science White Papers, 53, (2009).

Daly, R. A. and Loeb, A., ApJ., 364, 451, (1990).

David, L. P., Jones, C., \& Forman, W., ApJ, 445, 578, (1995).

Dawson, J. M., Adv. Plasma Phys., 1, 1, (1968).

Demarco, R., Magnard, F., Durret, F., \& Márquez, I., A\&A, 407, 437, (2003).

de Souza, R. S. and Opher, R., Phys. Rev. D77, 043529 (2008).

Dolag, K., Bartelmann, M., \& Lesch, H., A\&A, 348, 351, (1999).

Dolag, K. \& Schindler, S., A\&A, 364, 491, (2000).

Dolag, K., Evrard, A., \& Bartelmann, M., A\&A, 369, 36, (2001a).

Dolag, K., Schindler, S., Govoni, F., \& Feretti, L., A\&A, 378, 777, (2001b).

Dolag, K., Bartelmann, M., \& Lesch, H., A\&A, 387, 383, (2002).

Dolag, K., Grasso, D., Springel, V., \& Tkachev, I., JETP Lett, 79, 583, (2004).

Dolag, K., Grasso, D., Springel, V., \& Tkachev, I., JCAP, 01, 09, (2005).

Dolag, K., Vazza, F., Brunnetti, G., \& Tormen, G., MNRAS, 364, 753, (2005).

Dolgov, A., \& Silk, J., Phys. Rev. D, 47, 3144, (1993).

Dreher, J. W., Carilli, C. L., \& Perley, R. A., ApJ, 316, 611, (1987).

Duncan, R. C., Thompson, C., ApJ, L9, 392, (1992).

Durret, F., Lima Neto, G. B., \& Forman, W., A\&A,432, 809, (2005).

Efstathiou, G. \& Jones, B. J. T., MNRAS, 186, 133, (1979).

Enqvist, K., \& Olesen, P., Phys. Lett. B, 319, 178, (1993).

Enqvist, K. Rez, A. I. \& Semioz, V. B., Nucl. Phys. B, 436, 49, (1995).

Farrar, G. R., \& Piran, T., Phys. Rev. Lett.84, 3527, (2000). 
Feretti, L., Dallacasa, D., Govoni, F., et al., A\&A, 344, 472, (1999).

Feretti, L., \& Johnston-Hollitt, M., NewAR, 48, 1145, (2004).

Feretti, L., Orr'u, E., Brunetti, G., et al., A\&A, 423, 111, (2004).

Feretti, L., Dallacasa, D., Govoni, F., et al., A\&A, 344, 472, (1999).

Fermi, E., Phys. Rev., 75, 1169, (1949).

Finoguenov, A., Reiprich, T. H., \& Böhringer, H., A\&A, 368, 749, (2001).

Fukugita, M. \& Kawasaki, M., MNRAS, 343, L25, (2003).

Giovannini, G., Feretti, L., Venturi, T., Kim, K.-T.,\& Kronberg, P. P., ApJ, 406, 399, (1993).

Giovannini, M., New Astron. 5, 335, (2000).

Giovannini, M., Int. J. of Mod.Phys. D, 13, 391, (2004).

Gnedin, N. Y., Ferrara, A., \& Zweibel, E. G., ApJ, 539, 505, (2000).

Govoni, F., Enßlin, T. A., Feretti, L.,\& Giovannini, G., A\&A, 369, 441, (2001).

Govoni, F., Taylor, G. B., Dallacasa, D., Feretti, L., \& Giovannini, G., IAUJD 10, 16, (2002).

Govoni, F. \& Feretti, L., International Journal of Modern Physics D, 13, 1549, (2004).

Grasso, D., \& Rubinstein, H. R., Phys. Rep., 348, 163, (2001).

Grasso, D., \& Rubinstein, H. R., Phys. Lett. B, 379, 73, (1996).

Haiman, Z. \& Holder, G., P., ApJ, 595, 1, (2003).

Halcomb, K. A. \& Tajima, T., Phys. Rev. D, 40, 3809, (1989).

Hall, J. S., \& Mikesell, A. M. AJ, 54, 187, (1949).

Han, J. L., Wielebinski, R., Chinese Journal of Astronomy and Astrophysics, 2, 293, (2002).

Hiltner, W. A., ApJ, 109, 471, (1949).

Hindmarsh, M., Everett, A., Phys. Rev. D, 58, 103505, (1998).

Hogan, C. J., Phys. Rev. Lett., 51, 1488, (1983). 
Jackson, J. D., Classical Electrodynamics (John Wiley \& Sons, New York 1975).

Jafelice, L. C., Opher, R., Assis, A. S., \& Busnardo-Neto, J., ApJ, 348, 61, (1990).

Jafelice, L. C., Opher, R., MNRAS, 257, 135, (1992).

Jaffe, W., ApJ, 241, 925, (1980).

Jones, C. \& Forman, W., ApJ, 276, 38, (1984).

Kahniashvili, T., Maravin, Y., Kosowsky, A., astro-ph/0806.1876v1, (2008).

Kaluza, T., Sitzungsber. Preuss. Akad. Wiss., Math. Klasse, 10, 966, (1921).

Kang, H., Cen, R., Ostriker, J. P., \& Ryu, D., ApJ, 428, 1, (1994).

Katz, J. I., ApJ, 432, 107, (1994).

Kazantsev, A. P., Sov. Phys. JETP, 26, 1031, (1968).

Kim, K. T., Kronberg, P. P., Giovannini, G., \& Venturi, T. Nature, 341, 720, (1989).

Kim, K.-T., Kronberg, P. P., \& Tribble, P. C., ApJ, 379, 80, (1991).

Klein, O., Z. Phys., 37, 895, (1926).

Komatsu, E., Dunkley, J., Nolta, M. R.,Bennett, C. L., Gold, B., Hinshaw, G., Jarosik, N., Larson, D., Limon, M., Page, L., Spergel, D. N., Halpern, M., Hill, R. S.,Kogut, A., Meyer, S. S., Tucker, G. S., Weiland, J. L.,Wollack, E. \& Wright, E. L., ApJS, 180, 330, (2009).

Kritsuk \& Norman, ApJ, 580, 51, (2002).

Kronberg, P. P., Perry, J. J. \& Zukowski, E. L. H., ApJ Letters, 355, 31, (1990).

Kronberg, P. P., Rep. Prog. Phys., 57, 325, (1994).

Kubo, R., J. Phys. Soc. Japan, 12, 570, (1957).

Kulsrud, R. M., ARA\&A, 37, 37 (1999).

Kulsrud, R. M., Plasma Physics for Astrophysics(Princeton University Press, 2005)

Kulsrud, R. M. \& Zweibel, E. G., Rep. Prog. Phys. 71, 046901 (2008).

Lacey, C. \& Cole, S., MNRAS, 262, 627, (1993).

Laganá, T. F., Lima Neto, G. B., Andrade-Santos, F., \& Cypriano, E. S., A\&A, 485, 
633, (2008).

Landau, L. D., and Lifshitz, E. M., Fluid Mechanics (Oxford; Pergamon, 1987).

Laros, J. G., Fenimore, E. E., Fikani, M. M., Klebesadel, R. W., \& Barat, C., Nature, $322,152,(1986)$.

Lau, E. T., Kravtsov, A. V., Nagai, D., astro-ph/0903.4895, (2009).

Lazarian, A., Astronomische Nachrichten, 327, 609, (2006).

Lee, H. K.; Wijers, R. A. M. J. \&Brown, G. E., Phys. Rep., 325, 83, (2000).

Lei, W. H., Wang, D. X., Ma \& R. Y., Chin. J. Astron. Astrophys., 5, (2005).

Lei, W. H., Wang, D. X., Zou, Y. C. \& Lei, Z., Chin. J. Astron. Astrophys., 8, 404, (2008).

Lloyd, N. M., \& Petrosian, V. ApJ, 543, 722, (2000).

Loeb, A. \& Mao, S., ApJ, 435, L109, (1994).

Luchak, G., Can. J. Phys., 29, 470, (1952).

Malyshkin, L., \& Kulsrud, R. M., ApJ, 571, 619, (2002).

Markevitch, M.\& Vikhlinin, A., PhysicResp, 443, 1, (2007).

Matarrese, S., Mollerach, S., Notari, A. \& Riotto, A., Phys. Rev. D, 71, 043502, (2005).

Mereghetti, S. \& Stella, L., ApJ, 442, L17, (1995).

McComb, W. D., The Physics of Fluid Turbulence (Oxford: Clarendon 1990).

Medina-Tanco, G., Ensslin, T. A., Astropart. Phys., 16, 47, (2001).

Meszaros, P. , Rees, M. J., \& Wijers, R. A. M. J., ApJ, 499, 301, (1998).

Meszaros, P., Rep. Prog. Phys., 69, 2259, (2006).

Mikhail, F. I., Wanas, M. I. \& Eid, A. M., Ap\&SS, 228, 221, (1995).

Miranda, O. D. \& Opher, R., MNRAS, 283, 912, (1996).

Miranda, O. D. \& Opher, R., ApJ, 482, 573, (1997).

Miranda, O., Opher, M., \& Opher, R., MNRAS, 301, 547, (1998).

Modjaz, M., Moran,J.M. Greenhill, L.J. \& Kondratko, P.T., Magnetic Fields in the 
Accretion Disks of AGN: NGC 4258, in: Quasar Cores and Jets, 25th meeting of the IAU, Joint Discussion 18, Sydney, Australia, (2003).

Monin, A. S. \& Yaglom, A. A., Statistical Fluid Mechanics, (Vol. 2 Cambridge: MIT Press, 1975).

Morrison, P., Rev. Mod. Phys., 29, 235, (1957).

Naoz, S., Noter, S., Barkana, R., MNRAS, 376, 98, (2006).

Norman, M. L. \& Bryan, G. L., Lecture Notes in Physics, Berlin Springer Verlag, Vol. 530, The Radio Galaxy Messier 87, ed. H.-J. Röser \& K. Meisenheimer, 106, (1999).

Opher, M., \& Opher, R., Phys. Rev. Lett., 79, 2628, (1997a).

Opher, M., \& Opher, R., Phys. Rev. D, 56, 3296, (1997b).

Opher, M., \& Opher, R., Phys. Rev. D, 82, 4835, (1999).

Opher, R. \& Wichoski, U. F., Phys. Rev. Lett., 78, 787, (1997).

Padmanabhan, T., Structure Formation in the Universe (Cambridge: Univ. Press 1993).

Papapetrou, A., Philos. Mag., 41, 399, (1950).

Parker, E. N., J. Geophy. Res., 62, 509, (1957).

Parker, L., Phys. Rev. Lett., 21, 562, (1968).

Parker, E. N., ApJ, 163, 255 (1971).

Parker, E. N., Cosmical Magnetic Fields (Clarendon Press: Oxford 1979).

Passavano et al., ApJ, 584, 797, (2003).

Pauli, W., Ann. Phys., 18, 305, (1933).

Peebles, P. J. E., ApJ, 147, 859, (1967).

Peebles, P. J. E., ApJ, 155, 393, (1969).

Peebles, P. J. E., Principles of Physical Cosmology (Princeton: Princeton Univ. Press 1993).

Perley, R. A. \& Taylor, G. B., AJ, 101, 1623, (1991).

Perry, J. J., In Cosmical Magnetism. (ed. Lyndon-Bell, 144, 51, Cambridge 1994). 
Piffaretti, R. \& Valdarnini, R., arXiv:0808.1111, (2008).

Piran, T., Rev. Mod. Phys., 76, 1143, (2005).

Pislar, V., Durret, F., Gerbal, D., Lima Neto, G. B., \& Slezak, E., A\&A, 322, 53, (1997).

Pollack, L. K., Taylor, G. B., \& Allen, S. W., MNRAS, 359, 1229, (2005).

Popham R., Woosley S. E. \& Fryer C., ApJ, 518, 356, (1999).

Pudritz, R. E., \& Silk, J., ApJ, 342, 650, (1989).

Quashnock, J. M., Loeb, A., \& Spergel, D. N., ApJ, 344, L49, (1989).

Rasia, E., Tormen, G., \& Moscardini, L., 351, 237, MNRAS, (2004).

Rasia, E., Ettori, S., Moscardini, L., Mazzotta, P., Borgani, S., Dolag, K., Tormen, G., Cheng, L. M., \& Diaferio, A., MNRAS, 369, 2013, (2006).

Rees, M. J., \& Rheinhardt, M., A\&A, 19, 189, (1972).

Rees, M. J., QJRAS, 28, 197, (1987).

Rees, M. J., Cosmical Magnetism, ed. D.Lynden-Bell (Dordrecht: Kluwer, 155, 1994).

Reiprich, T. H. \& Böhringer, H., ApJ, 567, 716, (2002).

Ricker, P. M. \& Sarazin, C. L., ApJ, 561, 621, (2001).

Rosner, R. \& Deluca, E., IAUS, 136, 319, (1989).

Rostoker, N., Aamodt, R., \& Eldridge, O., Ann. Phys., 31, 243, (1965).

Ruzmaikin, A. A, Shukurov, A. M., \& Sokoloff, D. D., Magnetic Fields of Galaxies (Kluwer Academic publisher: Dordrecht 1988).

Radiative Process in Astrophysics, (Wiley-Interscience publication 1979).

Ryu, D., Kang, H., \& Biermann, P. L., A\&A, 335, 19, (1998).

Ryu, D., Ostriker, J. P., Kang, H., \& Cen, R., ApJ, 414, 1, (1993).

Ryu, D., Kang, H., Cho, J. \& Das, S., Science, 320, 909, (2008).

Sanchez-Salcedo, F. J., Brandenburg, A., \& Shukurov, A., ApSS, 263, 87, (1999).

Sarazin, C. L., Reviews of Modern Physics, 58, 1, (1986).

Sarazin, C. L., Astrophysics and Space Science Library, Vol. 272, Merging Processes in 
Galaxy Clusters, ed. L. Feretti, I. M. Gioia, \& G. Giovannini, 1-38, (2002).

Sari, R., Piran, T., \& Narayan, R., ApJ, 497, L17, (1998).

Schekochihin, A. A., Boldyrev, S. A., Kulsrud, R. M., ApJ, 567, 828, (2002).

Schuecker, P., Finoguenov, A., Miniati, F., B“ohringer, H., \& Briel, U. G., A\&A, 426, $387,(2004)$.

Schuster, A., Proc. R. Inst., 13, 273, (1980).

Shklovskij, I., Dokl. Akad. Nauk. USSR90, 983, (1953).

Shu, F. H., Gas Dynamics, V.II of The Physics of Astrophysics (University Science Books 1992).

Sigl, G., Olinto, A., \& Jedamkiz, K., Phys. Rev. D, 55, 4582, (1997).

Sigl, G., Miniati, F., \& Ensslin, T. A., Phys. Rev. D, 68, 043002, (2003).

Sirag, S. P., Nature, 278, 535, (1979).

Sitenko, A. G., Electromagnetic Fluctuations in Plasma (NY:Academic Press 1967).

Soker, N. \& Sarazin, C. L., ApJ, 348, 73, (1990).

Soltan, A. MNRAS, 200, 115, (1982).

Somerville, R., S., \& Livio, M., ApJ, 593, 611, (2003).

Spitzer Jr, L., Physics of fully ionized gases, (New York: Wiley, 1962).

Steenbeck, M., Krause, F., \& Rädler, K. H., Z. Naturforsch., 21a, 369, (1966).

Sturrock, P. A. , Plasma Physics (Cambridge: Cambridge Univ. Press 1994).

Subramanian, K., Narasimha, D., \& Chitre, S. M., MNRAS, 271, 15, (1994).

Subramanian, K., Phys. Rev. Lett., 83, 2957, (1999).

Subramanian, K., Astron. Nachr., 327, 403, (2006a).

Subramanian, K., Shukurov, A., \& Haugen, N. E. L., MNRAS, 366, 1437, (2006b).

Tajima, T., Sakai, J., Nakajima, H., Kosugi, T., Brunel, F., \& Kundu, M. R., ApJ, 321, 1031, (1987).

Tajima, T., Cable, S., Shibata, K., \& Kulsrud, R. M., ApJ, 390, 309, (1992). 
Tajima, T., Cable, S., \& Kulsrud, R. M., Phys. Fluids B, 4, 2338, (1992).

Takahashi, K., Ichiki, K., Ohno, H., \& Hanayama, H., Phys. Rev. Lett., 95, 121301, (2005).

Tashito, H. \& Sugiyama, N., MNRAS, 368, 965, (2006).

Taylor, G. B. \& Perley, R. A., ApJ, 416, 554, (1993).

Taylor, G. B., Barton, E. J., \& Ge, J., AJ, 107, 1942, (1994).

Taylor, G. B., Fabian, A. C., \& Allen, S. W., MNRAS, 334, 769, (2002).

Thompson, C., Duncan, R. C., MNRAS, 275, 255, (1995).

Thompson, C., Duncan, R. C., ApJ, 473, 322, (1996).

Turner, M. S., Widrow, L. M., Phys. Rev. D, 37, 2743, (1988).

Uzdensky, D. A., The Astronomy and Astrophysics Decadal Survey, Science White Papers, 302, (2009).

Vachaspati, T., Phys. Lett. B, 265, 258, (1991).

Vainshtein, S. I., \& Ruzmaikin, A. A., Sov. Astron., 15, 714, (1972).

Vainshtein S. I. \& Kichatinov, L. L., JFM, 168, 73, (1986).

Vallée, J. P., Fund. Cosm. Phys., 19, 1, (1997).

Vallée, J. P., New Astromy Reviews, 48, 763, (2004).

Vikhlinin, A., Kravtsov, A., Forman,W., et al., ApJ, 640, 691, (2006).

Vink, J., Kuiper, L., MNRAS, 370, 14, (2006).

Vogt, C., \& Ensslin, T. A., A\&A, 412, 373, (2003).

Volonteri, M. et al., ApJ, 620, 69, (2005).

Walsh, W., Beck, R., Thuma, G., et al., A\&A 388, 7, (2002).

Wang, J. M., Chen, Y. M., Ho, L. C., \& McLure, R. J., ApJ, 642, 10, (2006).

Wasserman, I., ApJ, 224, 337, (1978).

Weinberg, S., Cosmology (Oxford, University Press, 2008).

Westerhoff, S. et al., Nucl. Phys. B, 46, 136, (2004). 
White, S. D. M., ApJ, 286, 38, (1984).

White, D. A. \& Fabian, A. C., MNRAS, 273, 72, (1995).

Widrow, L. M., Rev. Mod. Phys., 74, 775, (2002).

Woodward, J. F., Found. Phys. 19, 1345, (1989).

Wu, X. P. \& Fang, L.-Z., ApJ, 483, 62, (1997).

Xue, Y. J. \& Wu, X.-P., MNRAS, 318, 715, (2000).

Zavala, R.T. \& Taylor, G.B., New Astron. Rev., 47, 589, (2003).

Zel'dovich, Y. B., Ruzmaikin, A. A., \& Sokoloff, D. D., Magnetic Fields in Astrophysics (New York: Gordon \& Breach 1983).

Zhang, P., MNRAS, 348, 1348, (2004).

Zweibel, G. \& Heiles, C., Nature 385, 131, (1997). 